TRANSACTIONS OF THE

AMERICAN MATHEMATICAL SOCIETY

Volume 359, Number 10, October 2007, Pages 4865-4943

S 0002-9947(07)04182-7

Article electronically published on May 16, 2007

\title{
A KOSZUL DUALITY FOR PROPS
}

\author{
BRUNO VALLETTE
}

\begin{abstract}
The notion of prop models the operations with multiple inputs and multiple outputs, acting on some algebraic structures like the bialgebras or the Lie bialgebras. In this paper, we generalize the Koszul duality theory of associative algebras and operads to props.
\end{abstract}

\section{INTRODUCTION}

The Koszul duality is a theory developed for the first time in 1970 by S. Priddy for associative algebras $[\mathrm{Pr}]$. To every quadratic algebra $A$, it associates a dual coalgebra $A^{i}$ and a chain complex called a Koszul complex. When this complex is acyclic, we say that $A$ is a Koszul algebra. In this case, the algebra $A$ and its representations have many properties ( $c f$. A. Beilinson, V. Ginzburg and W. Soergel [BGS]).

In 1994, this theory was generalized to algebraic operads by V. Ginzburg and M.M. Kapranov ( $c f$. GK]). An operad is an algebraic object that models the operations with $n$ inputs (and one output) $A^{\otimes n} \rightarrow A$ acting on a type of algebras. For instance, there exist operads $\mathcal{A} s, \mathcal{C}$ om and $\mathcal{L}$ ie coding associative, commutative and Lie algebras. The Koszul duality theory for operads has many applications: construction of a "small" chain complex to compute the homology groups of an algebra, minimal model of an operad, and notion of algebra up to homotopy.

The discovery of quantum groups ( $c f$. V. Drinfeld [Dr1, Dr2]) has popularized algebraic structures with products and coproducts, that's to say, operations with multiple inputs and multiple outputs $A^{\otimes n} \rightarrow A^{\otimes m}$. It is the case of bialgebras, Hopf algebras and Lie bialgebras, for instance. The framework of operads is too narrow to treat such structures. To model the operations with multiple inputs and outputs, one has to use a more general algebraic object: the props. Following J.-P. Serre in [S], we call an "algebra" over a prop $\mathcal{P}$, a $\mathcal{P}$-algebra.

It is natural to try to generalize Koszul duality theory to props. A few works have been done in that direction by M. Markl and A.A. Voronov in MV] and W.L. Gan in G. Actually, M. Markl and A.A. Voronov proved Koszul duality theory for what M. Kontsevich calls $\frac{1}{2}$-PROPs, and W.L. Gan proved it for dioperads. One has to bear in mind that an associative algebra is an operad, an operad is a $\frac{1}{2}$-PROP, a $\frac{1}{2}$-PROP is a dioperad and a dioperad induces a prop:

Associative algebras $\hookrightarrow$ Operads $\hookrightarrow \frac{1}{2}-$ PROPs $\hookrightarrow$ Dioperads $\leadsto$ Props.

Received by the editors June 27, 2005.

2000 Mathematics Subject Classification. Primary 18D50; Secondary 16W30, 17B26, 55P48.

Key words and phrases. Prop, Koszul duality, operad, Lie bialgebra, Frobenius algebra.

(C)2007 American Mathematical Society

Reverts to public domain 28 years from publication 4865 
In this article, we introduce the notion of properad, which is the connected part of a prop and contains all the information to code algebraic structure like bialgebras, Lie bialgebras, and infinitesimal Hopf algebras (cf. M. Aguiar Ag1, Ag2, Ag3 ). We prove the Koszul duality theory for properads which gives Koszul duality for quadratic props defined by connected relations (it is the case of the two last examples).

The first difficulty is to generalize the bar and cobar constructions to properads and props. The differentials of the bar and cobar constructions for associative algebras, operads, $\frac{1}{2}$-PROPs and dioperads are given by the notions of 'edge contraction' and 'vertex expansion' introduced by M. Kontsevich in the context of graph homology $(c f . \underline{\mathrm{Ko}})$. We define the differentials of the bar and cobar constructions of properads using conceptual properties. This generalizes the previous constructions.

The preceding proofs of Koszul duality theories are based on combinatorial properties of trees or graphs of genus 0. Such proofs cannot be used in the context of props since we have to work with any kind of graph. To solve this problem, we introduce an extra grading induced by analytic functors and generalize the comparison lemmas of B. Fresse $\mathrm{Fr}$ to properads.

To any quadratic properad $\mathcal{P}$, we associated a Koszul dual coproperad $\mathcal{P}^{i}$ and a Koszul complex. The main theorem of this paper is a criterion which claims that the Koszul complex is acyclic if and only if the cobar construction of the Koszul dual $\mathcal{P}^{i}$ is a resolution of $\mathcal{P}$. In this case, we say that the properad is Koszul and this resolution is the minimal model of $\mathcal{P}$.

As a corollary, we get the deformation theories for gebras over Koszul properads. For instance, we prove that the properad of Lie bialgebras and the properad of infinitesimal Hopf algebras are Koszul, which gives the notions of Lie bialgebra up to homotopy and infinitesimal Hopf algebra up to homotopy. Structures of gebras up to homotopy appear in the level of on some chain complexes. For instance, the Hochschild cohomology of an associative algebra with coefficients into itself has a structure of Gerstenhaber algebra up to homotopy ( $c f$. J.E. McClure and J.H. Smith [McCS, see also C. Berger and B. Fresse [BF]). This conjecture is known as Deligne's conjecture. The same kind of conjectures exist for gebras with coproducts up to homotopy. One of them was formulated by M. Chas and D. Sullivan in the context of String Topology ( $c f$. [CS] $)$. This conjecture says that the structure of involutive Lie bialgebra (a particular type of Lie bialgebras) on some homology groups can be lifted to a structure of involutive Lie bialgebras up to homotopy.

Working with complexes of graphs of genus greater than 0 is not an easy task. This Koszul duality for props provides new methods for studying the homology of these chain complexes. One can interpret the bar and cobar constructions of Koszul properads in terms of graph complexes. Therefore, it is possible to compute their (co)homology in Kontsevich's sense. For instance, the case of the properad of Lie bialgebras leads to the computation of the cohomology of classical graphs, and the case of the properad of infinitesimal Hopf algebras leads to the computation of the cohomology of ribbon graphs ( $c f$. M. Markl and A.A. Voronov [MV]). In these cases, the homology groups are given by the associated Koszul dual coproperad. So we get a complete description of them as $\mathbb{S}$-bimodules. In a different 
context, S. Merkulov recently gave another proof of the famous formality theorem of M. Kontsevich using the properties of chain complexes of Koszul props in [Me].

In the last section of this paper, we generalize the Poincaré series of associative algebras and operads to properads. When a properad $\mathcal{P}$ is Koszul, we prove a functional equation between the Poincaré series of $\mathcal{P}$ and the Poincaré series of its Koszul dual $\mathcal{P}$.

\section{Conventions}

Throughout the text, we will use the following conventions.

We work over a field $k$ of characteristic 0 .

0.1. $n$-tuples. To simplify the notations, we often write $\bar{\imath}$ an $n$-tuple $\left(i_{1}, \ldots, i_{n}\right)$. The $n$-tuples considered here are $n$-tuples of non-negative integers. We denote the sum $i_{1}+\cdots+i_{n}$ by $|\bar{\imath}|$. When there is no ambiguity about the number of terms, the $n$-tuple $(1, \ldots, 1)$ is denoted by $\overline{1}$.

We use the notation $\bar{\imath}$ to represent the "products" of elements indexed by the $n$-tuple $\left(i_{1}, \ldots, i_{n}\right)$. For instance, in the case of $k$-modules, $V_{\bar{\imath}}$ corresponds to $V_{i_{1}} \otimes_{k} \cdots \otimes_{k} V_{i_{n}}$

0.2. Partitions. A partition of an integer $n$ is an ordered increasing sequence $\left(i_{1}, \ldots, i_{k}\right)$ of non-negative integers such that $i_{1}+\cdots+i_{k}=n$.

We denote the set $\{1, \ldots, n\}$ by $[n]$. A partition of the set $[n]$ is a set $\left\{I_{1}, \ldots, I_{k}\right\}$ of disjoint subsets of $[n]$ such that $I_{1} \cup \cdots \cup I_{k}=[n]$.

0.3. Symmetric group and block permutations. We denote the symmetric group of permutations of $[n]$ by $\mathbb{S}_{n}$. Every permutation $\sigma$ of $\mathbb{S}_{n}$ is written with the $n$-tuple $(\sigma(1), \ldots, \sigma(n))$. We denote the subgroup $\mathbb{S}_{i_{1}} \times \cdots \times \mathbb{S}_{i_{n}}$ of $\mathbb{S}_{|\bar{\imath}|}$ by $\mathbb{S}_{\bar{\imath}}$. From every permutation $\tau$ of $\mathbb{S}_{n}$ and every $n$-tuple $\bar{\imath}=\left(i_{1}, \ldots, i_{n}\right)$, one associates a permutation $\tau_{\bar{\imath}}$ of $\mathbb{S}_{|\bar{\imath}|}$, called a block permutation of type $\bar{\imath}$, defined by

$$
\begin{aligned}
\tau_{\bar{\imath}}=\tau_{i_{1}, \ldots, i_{n}}:= & \left(i_{1}+\cdots+i_{\tau^{-1}(1)-1}+1, \ldots, i_{1}+\cdots+i_{\tau^{-1}(1)}, \ldots,\right. \\
& \left.i_{1}+\cdots+i_{\tau^{-1}(n)-1}+1, \ldots, i_{1}+\cdots+i_{\tau^{-1}(n)}\right) .
\end{aligned}
$$

0.4. Gebra. Following the article of J.-P. Serre $[\underline{S}]$, we call a gebra any algebraic structure like algebras, coalgebras, bialgebras, etc.

\section{Composition products}

In this section we describe an algebraic framework which is used to represent the operations with multiple inputs and multiple outputs acting on algebraic structures. We introduce composition products which correspond to the compositions of such operations.

\subsection{S-bimodules.}

Definition (S-bimodule). An $\mathbb{S}$-bimodule is a collection $(\mathcal{P}(m, n))_{m, n \in \mathbb{N}}$, of $k$ modules $\mathcal{P}(m, n)$ endowed with an action of the symmetric group $\mathbb{S}_{m}$ on the left and an action of the symmetric group $\mathbb{S}_{n}$ on the right such that these two actions are compatible. 
A morphism between two $\mathbb{S}$-bimodules $\mathcal{P}, \mathcal{Q}$ is a collection of morphisms $f_{m, n}$ : $\mathcal{P}(m, n) \rightarrow \mathcal{Q}(m, n)$ which commute with the action of $\mathbb{S}_{m}$ on the left and with the action of $\mathbb{S}_{n}$ on the right.

The $\mathbb{S}$-bimodules and their morphisms form a category denoted $\mathbb{S}$-biMod.

The $\mathbb{S}$-bimodules are used to code the operations acting on different types of gebras. The module $\mathcal{P}(m, n)$ represents the operations with $n$ inputs and $m$ outputs:

$$
\mathcal{P}(m, n) \otimes_{\mathbb{S}_{n}} A^{\otimes n} \rightarrow A^{\otimes m} .
$$

Definition (Reduced $\mathbb{S}$-bimodule). A reduced $\mathbb{S}$-bimodule is an $\mathbb{S}$-bimodule $(\mathcal{P}(m, n))_{m, n \in \mathbb{N}}$ such that $\mathcal{P}(m, 0)=\mathcal{P}(0, n)=0$, for every $m$ and $n$ in $\mathbb{N}$.

1.2. Composition product of $\mathbb{S}$-bimodules. We define a product in the category of $\mathbb{S}$-bimodules in order to represent to composition of operations.

Definition (Directed graphs). A directed graph is a non-planar graph where the orientations of the edges are given by a global flow (from the top to the bottom, for instance). We suppose that the inputs and the outputs of each vertex are labelled by integers $\{1, \ldots, n\}$. The global inputs and outputs of each graphs are also supposed to be indexed by integers. We denote the set of such graphs by $\mathcal{G}$.

Every graph studied in this article will be directed.

Definition (2-level graphs). When the vertices of a directed graph $g$ can be dispatched on two levels, we say that $g$ is a 2-level graph. In this case, we denote by $\mathcal{N}_{i}$ the set of vertices on the $i^{\text {th }}$ level. The set of such graphs is denoted by $\mathcal{G}^{2}$ (cf. Figure 1)

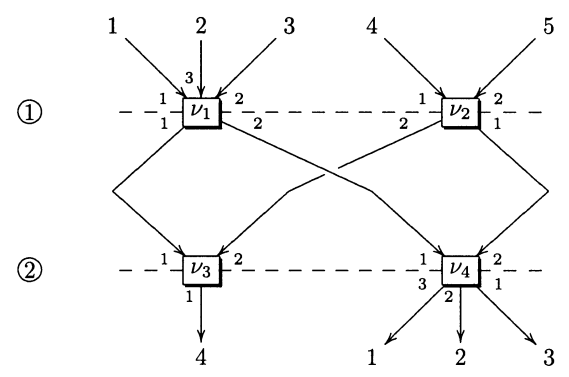

FiguRE 1. Example of a 2-level graph.

We can now define a product in the category of $\mathbb{S}$-bimodules based on 2-level graphs $\mathcal{G}^{2}$. We denote by $\operatorname{In}(\nu)$ and $\operatorname{Out}(\nu)$ the sets of inputs and outputs of a vertex $\nu$ of a graph.

Definition (Composition product $\otimes$ ). Given two $\mathbb{S}$-bimodules $\mathcal{P}$ and $\mathcal{Q}$, we define their product by the following formula:

$$
\mathcal{Q} \otimes \mathcal{P}:=\left(\bigoplus_{g \in \mathcal{G}^{2}} \bigotimes_{\nu \in \mathcal{N}_{2}} \mathcal{Q}(|\operatorname{Out}(\nu)|,|\operatorname{In}(\nu)|) \otimes_{k} \bigotimes_{\nu \in \mathcal{N}_{1}} \mathcal{P}(|\operatorname{Out}(\nu)|,|\operatorname{In}(\nu)|)\right) / \approx,
$$


where the equivalence relation $\approx$ is generated by

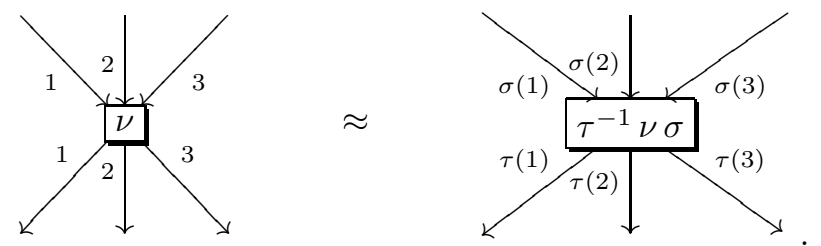

This product is given by the directed graphs on 2 levels where the vertices are indexed by elements of $\mathcal{Q}$ and $\mathcal{P}$ with respect to the inputs and outputs.

Remark. By concision, we will often omit the equivalence relation in the rest of the paper. It will always be implicit in the following that a graph indexed by elements of an $\mathbb{S}$-bimodule is considered with the equivalence relation.

This product has an algebraic writing using symmetric groups.

Given two $n$-tuples $\bar{\imath}$ and $\bar{\jmath}$, we use the following conventions. The notation $\mathcal{P}(\bar{\jmath}, \bar{\imath})$ denotes the product $\mathcal{P}\left(j_{1}, i_{1}\right) \otimes_{k} \cdots \otimes_{k} \mathcal{P}\left(j_{n}, i_{n}\right)$ and the notation $\mathbb{S}_{\bar{\imath}}$ denotes the image of the direct product of the groups $\mathbb{S}_{i_{1}} \times \cdots \times \mathbb{S}_{i_{n}}$ in $\mathbb{S}_{|\bar{\imath}|}$.

Theorem 1.1. Let $\mathcal{P}$ and $\mathcal{Q}$ be two $\mathbb{S}$-bimodules. Their composition product is isomorphic to the $\mathbb{S}$-bimodule given by the formula

$$
\mathcal{Q} \otimes \mathcal{P}(m, n) \cong \bigoplus_{N \in \mathbb{N}}\left(\bigoplus_{\bar{l}, \bar{k}, \bar{\jmath}, \bar{\imath}} k\left[\mathbb{S}_{m}\right] \otimes_{\mathbb{S}_{\bar{l}}} \mathcal{Q}(\bar{l}, \bar{k}) \otimes_{\mathbb{S}_{\bar{k}}} k\left[\mathbb{S}_{N}\right] \otimes_{\mathbb{S}_{\bar{\jmath}}} \mathcal{P}(\bar{\jmath}, \bar{\imath}) \otimes_{\mathbb{S}_{\bar{\imath}}} k\left[\mathbb{S}_{n}\right]\right) / \sim,
$$

where the direct sum runs over the b-tuples $\bar{l}, \bar{k}$ and the a-tuples $\bar{\jmath}, \bar{\imath}$ such that $|\bar{l}|=m,|\bar{k}|=|\bar{\jmath}|=N,|\bar{\imath}|=n$ and where the equivalence relation $\sim$ is defined by

$$
\begin{aligned}
& \theta \otimes q_{1} \otimes \cdots \otimes q_{b} \otimes \sigma \otimes p_{1} \otimes \cdots \otimes p_{a} \otimes \omega \\
& \sim \theta \tau_{\bar{l}}^{-1} \otimes q_{\tau^{-1}(1)} \otimes \cdots \otimes q_{\tau^{-1}(b)} \otimes \tau_{\bar{k}} \sigma \nu_{\bar{\jmath}} \otimes p_{\nu(1)} \otimes \cdots \otimes p_{\nu(a)} \otimes \nu_{\bar{\imath}}^{-1} \omega,
\end{aligned}
$$

for $\theta \in \mathbb{S}_{m}, \omega \in \mathbb{S}_{n}, \sigma \in \mathbb{S}_{N}$ and for $\tau \in \mathbb{S}_{b}$ with $\tau_{\bar{k}}$ the corresponding permutation by block (cf. Conventions), $\nu \in \mathbb{S}_{a}$ and $\nu_{\bar{\jmath}}$ the corresponding permutation by block.

Proof. To any element $\theta \otimes q_{1} \otimes \cdots \otimes q_{b} \otimes \sigma \otimes p_{1} \otimes \cdots \otimes p_{a} \otimes \omega$ of $k\left[\mathbb{S}_{m}\right] \otimes \mathcal{Q}(\bar{l}, \bar{k}) \otimes k\left[\mathbb{S}_{N}\right] \otimes$ $\mathcal{P}(\bar{\jmath}, \bar{\imath}) \otimes k\left[\mathbb{S}_{n}\right]$, we associate a graph $\Psi\left(\theta \otimes q_{1} \otimes \cdots \otimes q_{b} \otimes \sigma \otimes p_{1} \otimes \cdots \otimes p_{a} \otimes \omega\right)$ such that its vertices are indexed by the $q_{\beta}$ and the $p_{\alpha}$, for $1 \leq \beta \leq b$ and $1 \leq \alpha \leq a$. To do that, we consider a geometric representation of the permutation $\sigma$. We gather and index the outputs according to $\bar{k}$ and the inputs according to $\bar{\jmath}$. We index the vertices by the $q_{\beta}$ and the $p_{\alpha}$. Then, for each $q_{\beta}$, we build $l_{\beta}$ outgoing edges whose roots are labelled by $1, \ldots, l_{\beta}$. We do the same with the $p_{\alpha}$ and the inputs of the graph. Finally, we label the outputs of the graph according to $\theta$ and the inputs according to $\omega$. For instance, the element $(4123) \otimes q_{1} \otimes q_{2} \otimes(1324) \otimes p_{1} \otimes p_{2} \otimes(12435)$ gives the graph represented in Figure 2,

Let $\bar{\Psi}\left(\theta \otimes q_{1} \otimes \cdots \otimes q_{b} \otimes \sigma \otimes p_{1} \otimes \cdots \otimes p_{a} \otimes \omega\right)$ be the equivalence class of $\Psi\left(\theta \otimes q_{1} \otimes \cdots \otimes q_{b} \otimes \sigma \otimes p_{1} \otimes \cdots \otimes p_{a} \otimes \omega\right)$ for the relation $\approx$. Therefore, the application $\bar{\Psi}$ naturally induces an application from the quotient $k\left[\mathbb{S}_{m}\right] \otimes_{\mathbb{S}_{\bar{l}}} \mathcal{Q}(\bar{l}, \bar{k}) \otimes_{\mathbb{S}_{\bar{k}}} k\left[\mathbb{S}_{N}\right] \otimes_{\mathbb{S}_{\bar{j}}}$ $\mathcal{P}(\bar{\jmath}, \bar{\imath}) \otimes_{\mathbb{S}_{\bar{\imath}}} k\left[\mathbb{S}_{n}\right]$. The equivalence relation $\sim$ corresponds, via $\Psi$, to a (homeomorphic) rearrangement in space of the graphs. Therefore, the non-planar graph created by $\bar{\Psi}$ is invariant on the equivalent class of $\theta \otimes q_{1} \otimes \cdots \otimes q_{b} \otimes \sigma \otimes p_{1} \otimes \cdots \otimes p_{a} \otimes \omega$ 


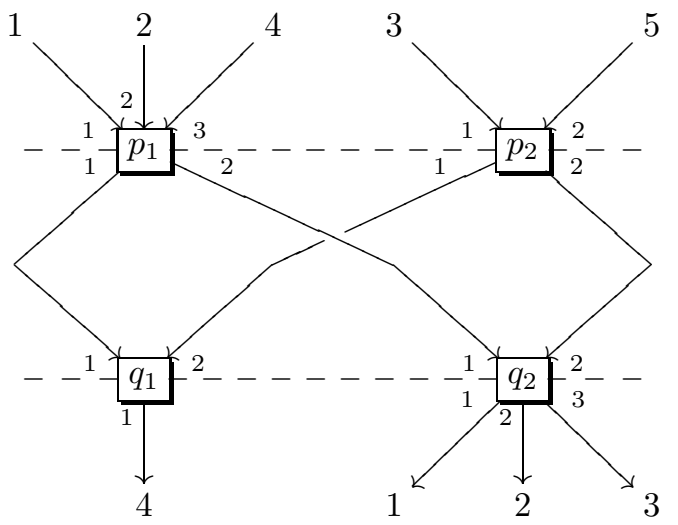

FIGURE 2. Image of $(4123) \otimes q_{1} \otimes q_{2} \otimes(1324) \otimes p_{1} \otimes p_{2} \otimes(12435)$ under $\Psi$.

under the relation $\sim$. This finally defines an application $\widetilde{\Psi}$ which is an isomorphism of $\mathbb{S}$-bimodules.

Proposition 1.2. The composition product $\otimes$ is associative. More precisely, let $\mathcal{R}, \mathcal{Q}$ and $\mathcal{P}$ be three $\mathbb{S}$-bimodules. There is a natural isomorphism of $\mathbb{S}$-bimodules

$$
(\mathcal{R} \otimes \mathcal{Q}) \otimes \mathcal{P} \cong \mathcal{R} \otimes(\mathcal{Q} \otimes \mathcal{P}) .
$$

Proof. Denote by $\mathcal{G}^{3}$ the set of 3-level graphs. The two $\mathbb{S}$-bimodules $(\mathcal{R} \otimes \mathcal{Q}) \otimes \mathcal{P}$ and $\mathcal{R} \otimes(\mathcal{Q} \otimes \mathcal{P})$ are isomorphic to the $\mathbb{S}$-bimodule given by the following formula:

$$
\begin{gathered}
\left(\bigoplus_{g \in \mathcal{G}^{3}} \bigotimes_{\nu \in \mathcal{N}_{3}} \mathcal{R}(|\operatorname{Out}(\nu)|,|\operatorname{In}(\nu)|) \otimes \bigotimes_{\nu \in \mathcal{N}_{2}} \mathcal{Q}(|\operatorname{Out}(\nu)|,|\operatorname{In}(\nu)|)\right. \\
\left.\otimes \bigotimes_{\nu \in \mathcal{N}_{1}} \mathcal{P}(|\operatorname{Out}(\nu)|,|\operatorname{In}(\nu)|)\right) / \approx
\end{gathered}
$$

\subsection{Horizontal and connected vertical composition products of $\mathbb{S}$-bimodules.}

Definition (Concatenation product $\otimes$ ). Let $\mathcal{P}$ and $\mathcal{Q}$ be two $\mathbb{S}$-bimodules. We define their concatenation product by the formula

$$
\begin{aligned}
& \mathcal{P} \otimes \mathcal{Q}(m, n):=\bigoplus_{\substack{m^{\prime}+m^{\prime \prime}=m \\
n^{\prime}+n^{\prime \prime}=n}} k\left[\mathbb{S}_{m^{\prime}+m^{\prime \prime}}\right] \otimes_{\mathbb{S}_{m^{\prime}} \times \mathbb{S}_{m^{\prime \prime}}} \mathcal{P}\left(m^{\prime}, n^{\prime}\right) \\
& \otimes_{k} \mathcal{Q}\left(m^{\prime \prime}, n^{\prime \prime}\right) \otimes_{\mathbb{S}_{n^{\prime}} \times \mathbb{S}_{n^{\prime \prime}}} k\left[\mathbb{S}_{n^{\prime}+n^{\prime \prime}}\right] .
\end{aligned}
$$

The product $\otimes$ corresponds to the intuitive notion of concatenation of operations (cf. Figure 3 ).

The concatenation product $\otimes$ is also called the horizontal product by contrast with the composition product $\otimes$, which is called the vertical product. 


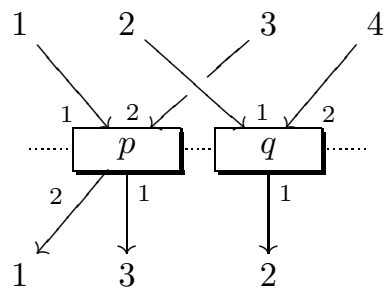

FiguRE 3. Concatenation of two operations.

Proposition 1.3. The concatenation product induces a symmetric monoidal category structure on the category of $\mathbb{S}$-bimodules. The unit is given by the $\mathbb{S}$-bimodule $k$ defined by

$$
\left\{\begin{array}{l}
k(0,0)=k, \\
k(m, n)=0 \quad \text { otherwise. }
\end{array}\right.
$$

Proof. The unit relation comes from

$$
\mathcal{P} \otimes k(m, n)=k\left[\mathbb{S}_{m}\right] \otimes_{\mathbb{S}_{m}} \mathcal{P}(m, n) \otimes_{k} k \otimes_{\mathbb{S}_{n}} k\left[\mathbb{S}_{n}\right] \cong \mathcal{P}(m, n),
$$

and the associativity relation comes from

$$
\begin{aligned}
& \left(\mathcal{P}(m, n) \otimes \mathcal{Q}\left(m^{\prime}, n^{\prime}\right)\right) \otimes R\left(m^{\prime \prime}, n^{\prime \prime}\right) \cong \mathcal{P}(m, n) \otimes\left(\mathcal{Q}\left(m^{\prime}, n^{\prime}\right) \otimes R\left(m^{\prime \prime}, n^{\prime \prime}\right)\right) \\
& \cong k\left[\mathbb{S}_{m+m^{\prime}+m^{\prime \prime}}\right] \otimes_{\mathbb{S}_{m} \times \mathbb{S}_{m^{\prime}} \times \mathbb{S}_{m^{\prime \prime}}} \mathcal{P}(m, n) \otimes_{k} \mathcal{Q}\left(m^{\prime}, n^{\prime}\right) \otimes_{k} R\left(m^{\prime \prime}, n^{\prime \prime}\right) \\
& \otimes_{\mathbb{S}_{n} \times \mathbb{S}_{n^{\prime}} \times \mathbb{S}_{n^{\prime \prime}}} k\left[\mathbb{S}_{n+n^{\prime}+n^{\prime \prime}}\right] \text {. }
\end{aligned}
$$

The symmetry isomorphism is given by the following formula:

$$
\begin{aligned}
\mathcal{P}(m, n) \otimes \mathcal{Q}\left(m^{\prime}, n^{\prime}\right) \rightarrow & (1,2)_{m, m^{\prime}}\left(\mathcal{P}(m, n) \otimes \mathcal{Q}\left(m^{\prime}, n^{\prime}\right)\right)(1,2)_{n, n^{\prime}} \\
& \cong \mathcal{Q}\left(m^{\prime}, n^{\prime}\right) \otimes \mathcal{P}(m, n) .
\end{aligned}
$$

Remark. The monoidal product $\otimes$ is bilinear. (It means that the functors $\mathcal{P} \otimes \bullet$ and $\bullet \otimes \mathcal{P}$ are additive for every $\mathbb{S}$-bimodule $\mathcal{P}$.)

The notions of $\mathbb{S}$-bimodules represents the operations acting on algebraic structures. The composition product $\nabla$ models their compositions. For some gebras, one can restrict to connected compositions, based on connected graphs, without loss of information ( $c f$. 2.9).

Definition (Connected graph). A connected graph $g$ is a directed graph which is connected as topological space. We denote the set of such graphs by $\mathcal{G}_{c}$.

Definition (Connected composition product $\bigotimes_{c}$ ). Given two $\mathbb{S}$-bimodules $\mathcal{Q}$ and $\mathcal{P}$, we define their connected composition product by the following formula:

$\mathcal{Q} \otimes_{c} \mathcal{P}:=\left(\bigoplus_{g \in \mathcal{G}_{c}^{2}} \bigotimes_{\nu \in \mathcal{N}_{2}} \mathcal{Q}(|O u t(\nu)|,|\operatorname{In}(\nu)|) \otimes_{k} \bigotimes_{\nu \in \mathcal{N}_{1}} \mathcal{P}(|\operatorname{Out}(\nu)|,|\operatorname{In}(\nu)|)\right) / \approx$.

In this case, the algebraic writing is more complicated. It involves a special class of permutations of the symmetric group $\mathbb{S}_{N}$. 
Definition (Connected permutations). Let $N$ be an integer. Let $\bar{k}=\left(k_{1}, \ldots, k_{b}\right)$ be a $b$-tuple and $\bar{\jmath}=\left(j_{1}, \ldots, j_{a}\right)$ be an $a$-tuple such that $|\bar{k}|=k_{1}+\cdots+k_{b}=|\bar{\jmath}|=$ $j_{1}+\cdots+j_{a}=N$. A $(\bar{k}, \bar{\jmath})$-connected permutation $\sigma$ of $\mathbb{S}_{N}$ is a permutation of $\mathbb{S}_{N}$ such that the graph of a geometric representation of $\sigma$ is connected if one gathers the inputs labelled by $j_{1}+\cdots+j_{i}+1, \ldots, j_{1}+\cdots+j_{i}+j_{i+1}$, for $0 \leq i \leq a-1$, and the outputs labelled by $k_{1}+\cdots+k_{i}+1, \ldots, k_{1}+\cdots+k_{i}+k_{i+1}$, for $0 \leq i \leq b-1$.

The set of $(\bar{k}, \bar{\jmath})$-connected permutations is denoted by $\mathbb{S}_{\bar{k}, \bar{\jmath}}^{c}$.

Example. Consider the permutation (1324) in $\mathbb{S}_{4}$ and the following geometric representation:

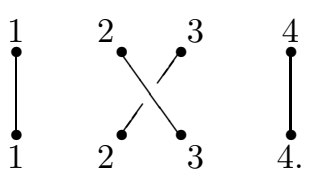

Take $\bar{k}=(2,2)$ and $\bar{\jmath}=(2,2)$. If one links the inputs 1,2 and 3,4 and the outputs 1,2 and 3,4 , it gives the following connected graph:

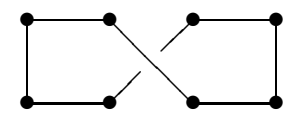

Therefore, the permutation $(1324)$ is $((2,2),(2,2))$-connected.

Counterexample. Consider the same permutation (1324) of $\mathbb{S}_{4}$ but let $\bar{k}=$ $(1,1,2)$ and $\bar{\jmath}=(2,1,1)$. One obtains the following non-connected graph:

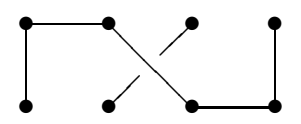

The next proposition will allow us to link the connected composition product with the one defined in the theory of operads.

Proposition 1.4. If $\bar{k}=(N)$, all the permutations of $\mathbb{S}_{N}$ are $((N), \bar{\jmath})$-connected, for every $\bar{\jmath}$. Otherwise stated, one has $\mathbb{S}_{(N), \bar{\jmath}}^{c}=\mathbb{S}_{N}$.

If $\bar{k}$ is different from $(N)$, one has $\mathbb{S}_{\bar{k},(1, \ldots, 1)}^{c}=\emptyset$.

Proof. The proof is obvious.

Proposition 1.5. Let $\mathcal{P}$ and $\mathcal{Q}$ be two $\mathbb{S}$-bimodules. Their connected composition product is isomorphic to the $\mathbb{S}$-bimodule given by the formula

$$
\begin{aligned}
\mathcal{Q} \otimes_{c} \mathcal{P}(m, n) \cong \\
\quad \bigoplus_{N \in \mathbb{N}}\left(\bigoplus_{\bar{l}, \bar{k}, \bar{\jmath}, \bar{\imath}} k\left[\mathbb{S}_{m}\right] \otimes_{\mathbb{S}_{\bar{l}}} \mathcal{Q}(\bar{l}, \bar{k}) \otimes_{\mathbb{S}_{\bar{k}}} k\left[\mathbb{S}_{\bar{k}, \bar{\jmath}}^{c}\right] \otimes_{\mathbb{S}_{\bar{\jmath}}} \mathcal{P}(\bar{\jmath}, \bar{\imath}) \otimes_{\mathbb{S}_{\bar{\imath}}} k\left[\mathbb{S}_{n}\right]\right) / \sim,
\end{aligned}
$$

where the direct sum runs over the $b$-tuples $\bar{l}, \bar{k}$ and the a-tuples $\bar{\jmath}, \bar{\imath}$ such that $|\bar{l}|=m,|\bar{k}|=|\bar{\jmath}|=N,|\bar{\imath}|=n$.

Proof. First, we have to prove that the right member is well defined. For $\bar{\jmath}$ a $a$-tuple and $\bar{k}$ a $b$-tuple such that $|\bar{\jmath}|=|\bar{k}|=N$, we denote $\bar{\jmath}^{\prime}:=\left(j_{\nu^{-1}(1)}, \ldots, j_{\nu^{-1}(b)}\right)$ and $\bar{k}^{\prime}:=\left(k_{\tau^{-1}(1)}, \ldots, k_{\tau^{-1}(b)}\right)$. They also verify $\left|\bar{\jmath}^{\prime}\right|=N$ and $\left|\bar{k}^{\prime}\right|=N$. Every $(\bar{k}, \bar{\jmath})-$ connected permutation $\sigma$ gives by composition on the left with a block permutation of the type $\tau_{\bar{k}}$ and by composition on the right with a bock permutation of the type 
$\nu_{\bar{\jmath}}$ a $\left(\bar{k}^{\prime}, \bar{\jmath}^{\prime}\right)$-connected permutation. The geometric representations of $\sigma \in \mathbb{S}_{N}$ and $\tau_{\bar{k}} \circ \sigma \circ \nu_{\bar{\jmath}}$ are homeomorphic and one links the same inputs and outputs. Therefore, if $\sigma \in \mathbb{S}_{\bar{k}, \bar{\jmath}}^{c}$, one has $\tau_{\bar{k}} \sigma \nu_{\bar{\jmath}} \in \mathbb{S}_{\bar{k}^{\prime}, \bar{\jmath}^{\prime}}^{c}$.

The rest of the proof uses the same arguments as the proof of Theorem 1.1 .

By opposition with the composition product $\otimes$, the connected composition product $\mathbb{}_{c}$ is a monoidal product in the category of $\mathbb{S}$-bimodules. It remains to define the unit in this category. We denote

$$
I:=\left\{\begin{array}{l}
I(1,1)=k, \\
I(m, n)=0 \quad \text { otherwise. }
\end{array}\right.
$$

Proposition 1.6. The category $\left(\mathbb{S}\right.$-biMod, $\left.\otimes_{c}, I\right)$ is a monoidal category.

Proof. To show the unit relation on $\mathcal{P} \bigotimes_{c} I(m, n)$, one has to study $\mathbb{S}_{\bar{k}, \bar{j}}^{c}$ in the case $\bar{\jmath}=(1, \ldots, 1)$. If one does not gather all the outputs, this set is empty. Otherwise, for $\bar{k}=(n)$, one has $\mathbb{S}_{(n),(1, \ldots, 1)}^{c}=\mathbb{S}_{n}(c f$. Proposition 1.4). This gives

$\mathcal{P} \otimes_{c} I(m, n)=\mathcal{P}(m, n) \otimes_{\mathbb{S}_{n}} k\left[\mathbb{S}_{n}\right] \otimes_{\mathbb{S}_{1}^{\times n}} k^{\otimes n} \cong \mathcal{P}(m, n) \otimes k . i d_{n} \otimes k^{\otimes n} \cong \mathcal{P}(m, n)$.

(The case $I \otimes_{c} \mathcal{P} \cong \mathcal{P}$ is symmetric.)

Once again, the associativity relation comes from the following formula

$$
\begin{aligned}
\mathcal{R} \otimes_{c}\left(\mathcal{Q} \otimes_{c} \mathcal{P}\right) \cong\left(\mathcal{R} \otimes_{c} \mathcal{Q}\right) \bigotimes_{c} \mathcal{P} & \\
\cong\left(\bigoplus_{g \in \mathcal{G}_{3}^{c}} \bigotimes_{\nu \in \mathcal{N}_{3}} \mathcal{R}(\mid \text { Out }(\nu)|,| \operatorname{In}(\nu) \mid)\right. & \otimes \bigotimes_{\nu \in \mathcal{N}_{2}} \mathcal{Q}(\mid \text { Out }(\nu)|,| \operatorname{In}(\nu) \mid) \\
& \left.\otimes \bigotimes_{\nu \in \mathcal{N}_{1}} \mathcal{P}(\mid \text { Out }(\nu)|,| \operatorname{In}(\nu) \mid)\right) / \approx .
\end{aligned}
$$

Examples. The monoidal categories $\left(k\right.$-Mod, $\left.\otimes_{k}, k\right)$ and $(\mathbb{S}-M o d, \circ, I)$ are two full monoidal subcategories of ( $\mathbb{S}$-biMod, $\left.\bigotimes_{c}, I\right)$.

The first category is the category of modules over $k$ endowed with the classical tensor product. Every vector space $V$ can be seen as the following $\mathbb{S}$-bimodule:

$$
\left\{\begin{array}{l}
V(1,1):=V \\
V(j, i):=0 \text { otherwise. }
\end{array}\right.
$$

One has $V \otimes_{c} W(1,1)=V \otimes_{k} W$. Since there exists no connected permutation associated to $a, b$-tuples of the form $(1, \ldots, 1)$ ( $c f$. Proposition 1.4), one has $V \otimes W(j, i)=0$ for $(j, i) \neq(1,1)$. And the morphisms between two $\mathbb{S}$-bimodules only composed by an $\left(\mathbb{S}_{1}, \mathbb{S}_{1}\right)$-module are exactly the morphisms of $k$-modules.

The category of $\mathbb{S}$-bimodules contains the category of $\mathbb{S}$-modules related to the theory of operads ( $c f$. [GK, L3, May $)$. Given an $\mathbb{S}$-module $\mathcal{P}$, we consider the following $\mathbb{S}$-bimodule:

$$
\begin{cases}\mathcal{P}(1, n):=\mathcal{P}(n) & \text { for } n \in \mathbb{N}^{*} \\ \mathcal{P}(j, i):=0 & \text { if } j \neq 1\end{cases}
$$


We have seen in Proposition 1.4 that $\mathbb{S}_{(N),(1, \ldots, 1)}^{c}=\mathbb{S}_{N}$. Here it gives

$\mathcal{Q} \otimes_{c} \mathcal{P}(1, n)=\bigoplus_{N \leq n}\left(\bigoplus_{i_{1}+\cdots+i_{N}=n} \mathcal{Q}(1, N) \otimes_{\mathbb{S}_{N}} k\left[\mathbb{S}_{N}\right] \otimes_{\mathbb{S}_{1}^{\times N}} \mathcal{P}(\overline{1}, \bar{\imath}) \otimes_{\mathbb{S}_{\bar{\imath}}} k\left[\mathbb{S}_{n}\right]\right) / \sim$,

where the equivalence relation $\sim$ is given by

$$
q \otimes \sigma \nu \otimes p_{1} \otimes \cdots \otimes p_{N} \sim q \otimes \sigma \otimes p_{\nu(1)} \otimes \cdots \otimes p_{\nu(N)} .
$$

It is equivalent to take the coinvariants under the action of $\mathbb{S}_{N}$ in the expression $\mathcal{Q}(1, N) \otimes_{k} \mathcal{P}\left(1, i_{1}\right) \otimes_{k} \cdots \otimes_{k} \mathcal{P}\left(1, i_{N}\right)$. Here we find the monoidal product defined in the category of $\mathbb{S}$-modules ( $c f$. GK, L3, May), which is well known under the following algebraic form (without the induced representations):

$\mathcal{Q} \otimes_{c} \mathcal{P}(1, n)=\bigoplus_{N \leq n}\left(\bigoplus_{i_{1}+\cdots+i_{N}=n} \mathcal{Q}(1, N) \otimes \mathcal{P}\left(1, i_{1}\right) \otimes \cdots \otimes \mathcal{P}\left(1, i_{N}\right)\right)_{\mathbb{S}_{N}}=\mathcal{Q} \circ \mathcal{P}(n)$.

Notice that the restriction of the monoidal product $\otimes_{c}$ on $\mathbb{S}$-modules corresponds to the description of the composition product o defined by trees ( $c f$. GK, L3]).

When $j \neq 1$, the composition $\mathcal{Q} \otimes_{c} \mathcal{P}(j, i)$ represents a concatenation of trees which is a non-connected graph. Therefore, $\mathcal{Q} \otimes_{c} \mathcal{P}(j, i)=0$, for $j \neq 1$.

The default of the composition product $\otimes$ to be a monoidal product, in the category of $\mathbb{S}$-bimodules, comes from the fact that $\mathcal{P} \otimes I$ corresponds to concatenations of elements of $\mathcal{P}$ and not only elements of $\mathcal{P}$.

Definition (The $\mathbb{S}$-bimodules $T_{\otimes}(\mathcal{P})$ and $\mathcal{S}(\mathcal{P})$ ). Since the monoidal product $\otimes$ is bilinear, the related free monoid on an $\mathbb{S}$-bimodule $\mathcal{P}$ is given by every possible concatenations of elements of $\mathcal{P}$

$$
T_{\otimes}(\mathcal{P}):=\bigoplus_{n \in \mathbb{N}} \mathcal{P}^{\otimes n}
$$

where $\mathcal{P}^{\otimes 0}=k$ by convention.

The monoidal product is symmetric. Therefore, we can consider the truncated symmetric algebra on $\mathcal{P}$ :

$$
\mathcal{S}(\mathcal{P}):=\bar{S}_{\otimes}(\mathcal{P})=\bigoplus_{n \in \mathbb{N}^{*}}\left(\mathcal{P}^{\otimes n}\right)_{\mathbb{S}_{n}} .
$$

The functor $\mathcal{S}$ allows us to link the two composition products $\square$ and $\bigotimes_{c}$.

Proposition 1.7. Let $\mathcal{P}$ and $\mathcal{Q}$ be two $\mathbb{S}$-bimodules. One has the following isomorphism of $\mathbb{S}$-bimodules:

$$
\mathcal{S}\left(\mathcal{Q} \otimes_{c} \mathcal{P}\right) \cong \mathcal{Q} \otimes \mathcal{P} .
$$

Proof. The isomorphism lies on the fact that every graph of $\mathcal{G}^{2}$ is the concatenation of connected graphs of $\mathcal{G}_{c}^{2}$, and the order between these connected graphs does not matter.

Remark that $\mathcal{P} \otimes I=\mathcal{S}(\mathcal{P})$, which is different from $\mathcal{P}$, in general.

Definition (Saturated $\mathbb{S}$-bimodules). A saturated $\mathbb{S}$-bimodule $\mathcal{P}$ is an $\mathbb{S}$-bimodule such that $\mathcal{P}=\mathcal{S}(\mathcal{P})$. We denote this category by sat-S-biMod.

Example. For every $\mathbb{S}$-bimodule $\mathcal{P}, \mathcal{S}(\mathcal{P})$ is a saturated $\mathbb{S}$-bimodule.

Notice that $\mathcal{S}(I)(m, n)=0$ if $m \neq n$ and $\mathcal{S}(n, n) \cong k\left[\mathbb{S}_{n}\right]$. 
Proposition 1.8. The category (sat- $\mathbb{S}-b i M o d, \bigotimes, \mathcal{S}(I)$ ) is a monoidal category.

Proof. Since $\mathcal{Q} \otimes \mathcal{P} \cong \mathcal{S}(\mathcal{Q} \otimes \mathcal{P})$, the associativity relation comes from Proposition 1.2. If $\mathcal{P}$ is a saturated $\mathbb{S}$-bimodule, one has $\mathcal{P} \otimes \mathcal{S}(I)=\mathcal{S}(\mathcal{P})=\mathcal{P}$.

We sum up these results in the diagram

$$
\left.\left(\text { Vect }, \otimes_{k}, k\right) \hookrightarrow(\mathbb{S}-M o d, \circ, I) \hookrightarrow\left(\mathbb{S} \text {-biMod, } \bigotimes_{c}, I\right) \stackrel{\mathcal{S}}{\longrightarrow} \text { (sat-S-biMod, } \otimes, \mathcal{S}(I)\right),
$$

where the first arrows are faithful functors of monoidal categories.

Remark. Since the concatenation product is symmetric and bilinear, its homological properties are well known. In the rest of the text, we will mainly study the homological properties of the composition products ( $c f$. Sections 3 and 5). Since the composition product $\nabla$ is obtained by concatenation $\mathcal{S}$ from the connected composition product $\nabla_{c}$, we will only state the theorems for the connected composition product $\otimes_{c}$ in the following text. There exist analogue theorems for the composition product $\otimes$, which are left to the reader.

1.4. Representation of the elements of $\mathcal{Q} \bigotimes_{c} \mathcal{P}$. The product $\mathcal{Q} \bigotimes_{c} \mathcal{P}$ (and $\mathcal{Q} \otimes \mathcal{P}$ ) is isomorphic to a quotient under the equivalence relation $\sim$ that permutates the order of the elements of $\mathcal{Q}$ and $\mathcal{P}$. For instance, to study the homological properties of this product, we need to find natural representatives of the classes of equivalence for the relation $\sim$.

In this section, all the $\mathbb{S}$-bimodules are reduced ( $c f$. 1.1).

Definition $\left(\mathbb{S}_{i, \nu}^{B}\right)$. We denote by $\mathbb{S}_{i, \nu}^{B}$ the set of block permutations of type $\underbrace{(i, \ldots, i)}_{\nu \text { times }}$ of $\mathbb{S}_{i \nu}$ (cf. Conventions).

Remark. The set $\mathbb{S}_{i, \nu}^{B}$ is a subgroup of $\mathbb{S}_{i \nu}$.

Let $\bar{\imath}$ be a partition of the integer $n$. We consider the $n$-tuple $\bar{\imath}^{*}$ defined by $i_{k}^{*}:=\left|\left\{j / i_{j}=k\right\}\right|$. Denote by $\mathbb{S}_{\bar{\imath}}^{B}$ the subgroup image of $\prod_{k=1}^{n} \mathbb{S}_{k, i_{k}^{*}}^{B}$ in $\mathbb{S}_{n}$.

Proposition 1.9. The product $\mathcal{Q} \otimes_{c} \mathcal{P}(m, n)$ is isomorphic to the $\mathbb{S}$-bimodule defined by the following formula:

$$
\bigoplus_{\Theta} k\left[\mathbb{S}_{m} / \mathbb{S}_{\bar{l}}^{B}\right] \otimes_{\mathbb{S}_{\bar{l}}} \mathcal{Q}(\bar{l}, \bar{k}) \otimes_{\mathbb{S}_{\bar{k}}} k\left[\mathbb{S}_{\bar{k}, \bar{\jmath}}^{c}\right] \otimes_{\mathbb{S}_{\bar{\jmath}}} \mathcal{P}(\bar{\jmath}, \bar{\imath}) \otimes_{\mathbb{S}_{\bar{\imath}}} k\left[\mathbb{S}_{\bar{\imath}}^{B} \backslash \mathbb{S}_{n}\right]
$$

where the sum $\Theta$ runs over $\bar{\imath}$ partition of the integer $n, \bar{l}$ partition of the integer $m$, $N$ in $\mathbb{N}^{*}$ and $\bar{\jmath}$ a-tuple, $\bar{k}$ b-tuple such that $|\bar{k}|=|\bar{\jmath}|=N$.

Proof. After choosing the order of the elements of $\mathcal{P}$ given by the partition $\bar{\imath}$ of the integer $n$, the remaining relations that permutate the elements of $\mathcal{P}$ involve only elements of $\mathbb{S}_{\bar{\imath}}^{B}$.

We can do the same kind of work with the definition of $\otimes_{c}$ using non-planar graphs. The goal here is to choose a planar representative of indexed graphs.

Definition (Ordered partitions of $[n]$ ). An ordered partition of $[n]$ is a sequence $\left(\Pi_{1}, \ldots, \Pi_{k}\right)$ such that $\left\{\Pi_{1}, \ldots, \Pi_{k}\right\}$ is a partition of $[n]$ and such that $\min \left(\Pi_{1}\right)<$ $\min \left(\Pi_{2}\right)<\cdots<\min \left(\Pi_{k}\right)$. 
Let $I$ be a set of $i$ elements. We denote by $\mathcal{P}(j, i)(I)$ the module

$$
\bigoplus_{f:[i] \rightarrow I} \mathcal{P}(j, i) / \asymp,
$$

where $f$ is a bijection from $[i]$ to $I$. Every element of $\mathcal{P}(j, i)(I)$ can be represented by a pair $(x, f)$. The equivalence relation $\asymp$ is defined by $(x . \tau, f) \asymp(x, f \circ \tau)$ for $\tau$ in $\mathbb{S}_{i}$. Therefore, $\mathcal{P}(j, i)(I)$ is an $\left(\mathbb{S}_{j}, \mathbb{S}_{I}\right)$-module. The module $\mathcal{P}(j, i)(I)$ represents the vertices indexed by an element of $\mathcal{P}(j, i)$ with $i$ inputs labelled by elements of $I$.

Proposition 1.10. The product $\mathcal{Q} \bigotimes_{c} \mathcal{P}(m, n)$ is isomorphic, as an $\mathbb{S}$-bimodule, to

$$
\begin{aligned}
\bigoplus_{\Theta^{\prime}} & \left(\left(\Pi_{1}^{\prime}\right) \mathcal{Q}\left(\left|\Pi_{1}^{\prime}\right|, k_{1}\right) \otimes_{k} \cdots \otimes_{k}\left(\Pi_{b}^{\prime}\right) \mathcal{Q}\left(\left|\Pi_{b}^{\prime}\right|, k_{b}\right)\right) \otimes_{\mathbb{S}_{\bar{k}}} k\left[\mathbb{S}_{\bar{k}, \bar{\jmath}}^{c}\right] \\
& \otimes_{\mathbb{S}_{\bar{j}}}\left(\mathcal{P}\left(j_{1},\left|\Pi_{1}\right|\right)\left(\Pi_{1}\right) \otimes_{k} \cdots \otimes_{k} \mathcal{P}\left(j_{a},\left|\Pi_{a}\right|\right)\left(\Pi_{a}\right)\right),
\end{aligned}
$$

where the sum $\Theta^{\prime}$ runs over pairs $\left(\left(\Pi_{1}^{\prime}, \ldots, \Pi_{b}^{\prime}\right),\left(\Pi_{1}, \ldots, \Pi_{a}\right)\right)$ of ordered partitions of $([m],[n]), N$ in $\mathbb{N}^{*}$ and $\bar{\jmath}$ a-tuple, $\bar{k}$ b-tuple such that $|\bar{k}|=|\bar{\jmath}|=N$.

As a consequence, we will often write the elements of $\mathcal{Q} \otimes_{c} \mathcal{P}$ as

$$
q_{1}^{\Pi_{1}^{\prime}} \otimes \cdots \otimes q_{b}^{\Pi_{b}^{\prime}} \otimes \sigma \otimes p_{1}^{\Pi_{1}} \otimes \cdots \otimes p_{a}^{\Pi_{a}}=\left(q_{1}^{\Pi_{1}^{\prime}}, \ldots, q_{b}^{\Pi_{b}^{\prime}}\right) \sigma\left(p_{1}^{\Pi_{1}}, \ldots, p_{a}^{\Pi_{a}}\right),
$$

or even without the П's.

Remark. The first operation $p_{1}$ has one input indexed by 1 , and the operation $q_{1}$ has one output indexed by 1 . We will use this property to build a contracting homotopy for the augmented bar and cobar construction (cf. Section 4.3).

The same propositions can be proved for the composition product $\otimes$. One has to change the set of connected permutations $\mathbb{S}_{\bar{k}, \bar{j}}^{c}$ by the symmetric group $\mathbb{S}_{N}$.

\section{DEFINITIONS OF PROPERAD AND PROP}

In this section, we give the definitions of properad and prop. These notions model the operations acting on gebras. We give the construction of the free properad and prop. Therefore, we can define the notions of quadratic properad and prop and give examples.

\subsection{Definition of properad.}

Definition (Properad). A properad is a monoid $(\mathcal{P}, \mu, \eta)$ in the monoidal category (S-biMod, $\left.\bigotimes_{c}, I\right)$. Equivalently, one defines a properad by

- An associative composition $\mathcal{P} \otimes_{c} \mathcal{P} \stackrel{\mu}{\rightarrow} \mathcal{P}$,

- and a unit $I \stackrel{\eta}{\rightarrow} \mathcal{P}$.

\section{Examples.}

- The inclusions of monoidal categories

$$
\left(\text { Vect, } \otimes_{k}, k\right) \hookrightarrow(\mathbb{S} \text {-Mod, } \circ, I) \hookrightarrow\left(\mathbb{S} \text {-biMod, } \otimes_{c}, I\right)
$$

show that an associative algebra is an operad and that an operad is a properad. 
- The fundamental example of properad is given by the $\mathbb{S}$-bimodule $\operatorname{End}(V)$ of multilinear applications of a vector space $V$ ( $c f$. 2.4).

- The main examples of properads studied here are the free and quadratic properads (cf. 2.7, 2.9).

Definition (Augmented properad). A properad $(\mathcal{P}, \mu, \eta, \epsilon)$ is called augmented if the counit (or augmentation map) $\epsilon: \mathcal{P} \rightarrow I$ is a morphism of properads.

Examples. The free properad $\mathcal{F}(V)(c f$. 2.7) and the quadratic properads (cf. 2.9) are augmented properads.

When $\mathcal{P}$ is an augmented properad, we have $\mathcal{P}=I \oplus \overline{\mathcal{P}}$, where $\overline{\mathcal{P}}$ is the kernel of $\epsilon$ and is called the ideal of augmentation.

Definition (Weight graded $\mathbb{S}$-bimodule). A weight graded $\mathbb{S}$-bimodule $M$ is a direct sum, indexed by $\rho \in \mathbb{N}$, of $\mathbb{S}$-bimodules $M=\bigoplus_{\rho \in \mathbb{N}} M^{(\rho)}$.

The morphisms of weight graded $\mathbb{S}$-bimodules are the morphisms of $\mathbb{S}$-bimodules that preserve this decomposition. The set of weight graded $\mathbb{S}$-bimodules with its morphisms forms a category denoted gr- $\mathbb{S}$-biMod.

One can generalize the various products of $\mathbb{S}$-bimodules to the weight graded framework. For instance, one has that $\left(\mathcal{Q} \otimes_{c} \mathcal{P}\right)^{(\rho)}$ is given by the sum on 2-level connected graphs indexed by elements of $\mathcal{Q}$ and $\mathcal{P}$ such that the total sum of the weight of these elements is equal to $\rho$.

Definition (Weight graded properad). A weight graded properad is a monoid in the monoidal category of weight graded $\mathbb{S}$-bimodules with the composition product $\otimes_{c}$.

A weight graded properad $\mathcal{P}$ such that $\mathcal{P}^{(0)}=I$ is called a connected properad.

\subsection{Definition of prop.}

Definition (Prop). A prop $(\mathcal{P}, \tilde{\mu}, \tilde{\eta})$ is a monoid in the monoidal category (sat- $\mathbb{S}$ biMod, $\nabla, \mathcal{S}(I))$. In other words,

- The $\mathbb{S}$-bimodule $\mathcal{P}$ is stable under concatenation $\mathcal{P} \otimes \mathcal{P} \hookrightarrow \mathcal{P}$.

- The composition $\mathcal{P} \otimes \mathcal{P} \stackrel{\mu}{\rightarrow} \mathcal{P}$ is associative.

- The morphism $\mathcal{S}(I) \stackrel{\eta}{\rightarrow} \mathcal{P}$ is a unit.

Remark. This definition corresponds to a $k$-linear version of the notion of PROP (cf. S. MacLane MacL2 and F.W. Lawvere La]). A prop is the support of the operations acting on algebraic structures. That's why we denote it with small letters. The name "properad" is a contraction of "prop" and "operad".

If one restricts the compositions of a prop to a connected graph, it gives a properad. Therefore, one can consider the forgetful functor $U_{c}$ from Props to Properads

$$
U_{c}: \text { Props } \rightarrow \text { Properads. }
$$


Proposition 2.1. The functor $\mathcal{S}$ induces a functor from Properads to Props. It is the left adjoint to the forgetful functor $U_{c}$ :

$$
\text { Props } \underset{\mathcal{S}}{\stackrel{U_{c}}{\rightleftharpoons}} \text { Properads. }
$$

Proof. Let $(\mathcal{P}, \mu, \eta)$ be a properad. We have seen that the $\mathbb{S}$-bimodule $\mathcal{S}(\mathcal{P})$ is saturated. The composition of the $\operatorname{prop} \mathcal{S}(\mathcal{P})$ is given by

$$
\mathcal{S}(\mathcal{P}) \otimes \mathcal{S}(\mathcal{P}) \cong \mathcal{S}\left(\mathcal{P} \otimes_{c} \mathcal{P}\right) \stackrel{\mathcal{S}(\mu)}{\longrightarrow} \mathcal{S}(\mathcal{P})
$$

and the unit comes from

$$
\mathcal{S}(I) \stackrel{\mathcal{S}(\eta)}{\longrightarrow} \mathcal{S}(\mathcal{P}) .
$$

The adjunction relation is left to the reader.

2.3. Definition of coproperad. Dually, we define the notion of coproperad.

Definition (Coproperad). A coproperad is a comonoid $(\mathcal{C}, \Delta, \varepsilon)$ in the monoidal category $\left(\mathbb{S}\right.$-biMod, $\left.\bigotimes_{c}, I\right)$. A structure of coproperad on $\mathcal{C}$ is given by

- A coassociative coproduct $\mathcal{C} \stackrel{\Delta}{\longrightarrow} \mathcal{C} \otimes_{c} \mathcal{C}$,

- and a counit $\mathcal{C} \stackrel{\varepsilon}{\rightarrow} I$.

Examples. The main examples of coproperads studied in this article are the cofree connected coproperad $\mathcal{F}^{c}(V)(c f$. 2.8) and the Koszul dual $\mathcal{P}$ i of a properad $\mathcal{P}(c f$. 7.1.1).

\subsection{The example of $\operatorname{End}(V)$.}

Definition $(\operatorname{End}(V))$. We denote by $\operatorname{End}(V)$ the set \{linear applications : $V^{\otimes n} \rightarrow$ $\left.V^{\otimes m}\right\}_{n, m}$. The symmetric groups $\mathbb{S}_{n}$ and $\mathbb{S}_{m}$ act on $V^{\otimes n}$ and $V^{\otimes m}$ by permutation of the variables. Therefore, $\operatorname{End}(V)$ is an $\mathbb{S}$-bimodule. The composition $\operatorname{End}(V) \otimes \operatorname{End}(V) \stackrel{\chi}{\rightarrow} \operatorname{End}(V)$ is given by the composition of linear applications. And the inclusion $\eta: \mathcal{S}(I) \hookrightarrow \operatorname{End}(V)$ corresponds to linear applications $V^{\otimes n} \rightarrow V^{\otimes n}$ obtained by permutation of the variables.

For instance, let $p_{\alpha} \in H o m_{k-\operatorname{Mod}}\left(V^{\otimes i_{\alpha}}, V^{\otimes j_{\alpha}}\right)$ and $q_{\beta} \in H o m_{k-\operatorname{Mod}}\left(V^{\otimes k_{\beta}}, V^{\otimes l_{\beta}}\right)$. The morphism $\chi\left(\theta \otimes q_{1} \otimes \cdots \otimes q_{b} \otimes \sigma \otimes p_{1} \otimes \cdots \otimes p_{a} \otimes \omega\right) \in H_{o m}$-Mod $\left(V^{\otimes n}, V^{\otimes m}\right)$ corresponds to the composition

$$
V^{\otimes n} \stackrel{\omega}{\longrightarrow} V^{\otimes n} \stackrel{p_{1} \otimes \cdots \otimes p_{a}}{\longrightarrow} V^{\otimes N} \stackrel{\sigma}{\longrightarrow} V^{\otimes N} \stackrel{q_{1} \otimes \cdots \otimes q_{b}}{\longrightarrow} V^{\otimes m} \stackrel{\theta}{\longrightarrow} V^{\otimes m},
$$

where the morphism $p_{1} \otimes \cdots \otimes p_{a}: V^{\otimes n}=V^{\otimes i_{1}} \otimes \cdots \otimes V^{\otimes i_{b}} \rightarrow V^{\otimes j_{1}} \otimes \cdots \otimes V^{\otimes j_{b}}=$ $V^{\otimes N}$ is the concatenation of morphisms $p_{\alpha}$.

The $\mathbb{S}$-bimodule $\operatorname{End}(V)$ is saturated and $(\operatorname{End}(V), \chi, \eta)$ is a prop. Its restriction to connected compositions $\operatorname{End}(V) \bigotimes_{c} \operatorname{End}(V) \stackrel{\chi_{c}}{\longrightarrow} \operatorname{End}(V)$ induces a structure of properad.

2.5. P-module. Here we recall the basic definitions related to the notion of module over a monoid that will be applied to properads and props throughout the text. For more details, we refer the reader to the book of S. MacLane MacL1]. 
A structure of module (on the right) $L$ over a monoid $\mathcal{P}$ in a monoidal category is given by a morphism $L \mathbb{\Xi}_{c} \mathcal{P} \stackrel{\rho}{\rightarrow} \mathcal{P}$ such that the following diagrams commute:

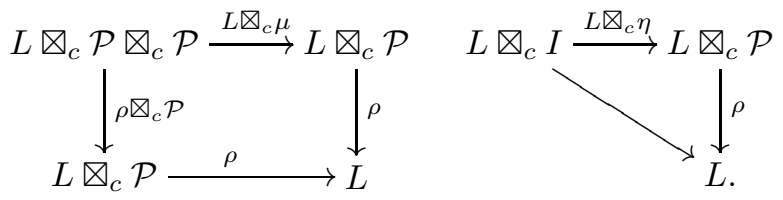

In this case, the object $L$ is called a $\mathcal{P}$-module (on the right).

The free module on the right on an object $M$ is given by $L:=M \otimes_{c} \mathcal{P}$. When $\mathcal{P}$ is a prop, the free $\mathcal{P}$-module on a saturated $\mathbb{S}$-module $M$ is $M \otimes \mathcal{P}$. In the rest of the text, we will call the free $\mathcal{P}$-module on an $\mathbb{S}$-bimodule $M$, the following $\mathcal{P}$-module $\mathcal{S}(M) \otimes \mathcal{P}=M \otimes \mathcal{P}$.

Definition (Relative composition product). Let $(L, \rho)$ be a right $\mathcal{P}$-module and $(R, \lambda)$ be a left $\mathcal{P}$-module. Their relative composition product $L \square_{c \mathcal{P}} R$ is given by the cokernel of the following maps:

$$
L \bigotimes_{c} \mathcal{P} \bigotimes_{c} R \underset{L \bigotimes_{c} \lambda}{\stackrel{\rho \bigotimes_{c} R}{\longrightarrow}} L \bigotimes_{c} R .
$$

When $(\mathcal{P}, \mu, \eta, \epsilon)$ is an augmented monoid, one can define a (left) action of $\mathcal{P}$ on $I$ by the formula

$$
\lambda_{\epsilon}: \mathcal{P} \bigotimes_{c} I \stackrel{\epsilon \bigotimes_{c} I}{\longrightarrow} I \bigotimes_{c} I \cong I .
$$

Definition (Indecomposable quotient). Let $(\mathcal{P}, \mu, \eta, \epsilon)$ be an augmented monoid in a monoidal category and let $(L, \rho)$ be a right $\mathcal{P}$-module. The indecomposable quotient of $L$ is the relative composition product of $L$ and $I$, that's to say, the cokernel of the maps

$$
L \bigotimes_{c} \mathcal{P} \bigotimes_{c} I \underset{L \bigotimes_{c} \lambda_{\epsilon}}{\stackrel{\rho}{\longrightarrow}} L \bigotimes_{c} I \cong L .
$$

When $(\mathcal{P}, \mu, \eta, \epsilon)$ is an augmented monoid, one has that the indecomposable quotient of the free module $L=M \bigotimes_{c} \mathcal{P}$ is isomorphic to $M$.

2.6. $\mathcal{P}$-gebra. The notion of $\mathcal{P}$-gebra is the natural generalization to the notion of an algebra over an operad.

Definition ( $\mathcal{P}$-gebra). Let $(\mathcal{P}, \mu, \eta)$ be a properad (or a prop). A structure of $\mathcal{P}$-gebra on a $k$-module $V$ is given by a morphism of properads (or props) : $\mathcal{P} \rightarrow$ $\operatorname{End}(V)$.

Remark. A $\mathcal{P}$-gebra is an "algebra" over a prop $\mathcal{P}$. The term "algebra" is to be taken here in the largest sense. For instance, a $\mathcal{P}$-gebra can be equipped with coproducts (operations with multiple outputs). It is the case for bialgebras, and Lie bialgebras.

Consider the $\mathbb{S}$-module $T(V)$ defined by $T(V)(n):=V^{\otimes n}$, where the (left) action is given by the permutation of variables. One can see that a morphism of (left) 
$\mathbb{S}$-modules $\mu_{V}: \mathcal{P} \otimes V \rightarrow T(V)$ induces a unique morphism of $\mathbb{S}$-modules $\widetilde{\mu_{V}}:$ $\mathcal{P} \otimes T(V) \rightarrow T(V)$. For instance, the element

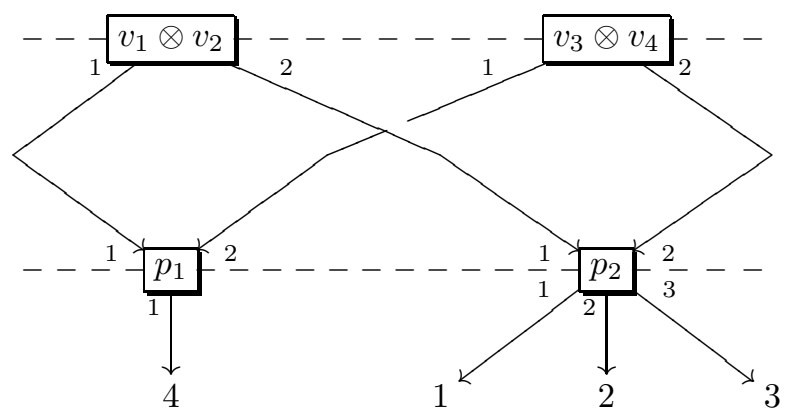

can be written

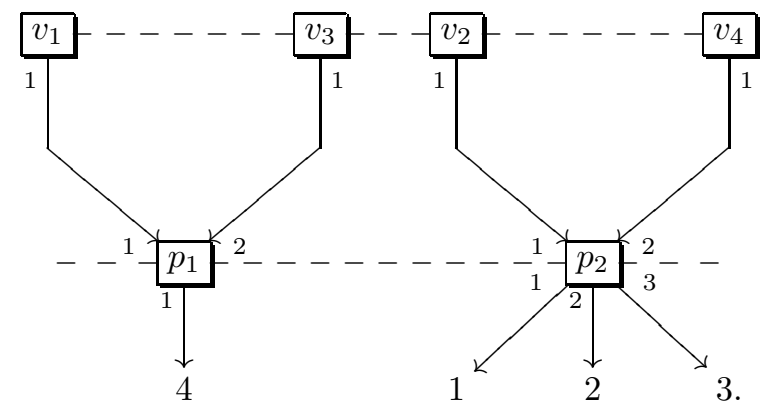

Proposition 2.2. A $\mathcal{P}$-gebra structure on a $k$-module $V$ is equivalent to a morphism $\mu_{V}: \mathcal{P} \otimes V \rightarrow T(V)$ such that the following diagram commutes:

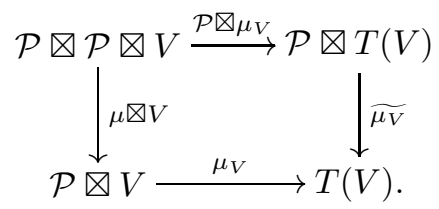

Remark. The same arguments hold for a gebra over a properad. When $\mathcal{P}$ is an operad, we find the classical notion of an algebra over an operad.

2.7. Free properad and free prop. In this section, we complete the following diagram of forgetful and left adjunct functors:

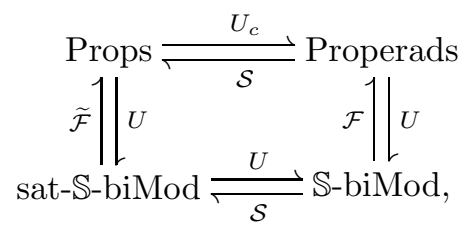

with the description of the functors $\mathcal{F}$ and $\widetilde{\mathcal{F}}$. To do this, we will use the construction of the free monoid described in $\mathrm{V} 1$.

We recall the construction of the free properad given in [V1] (section 5). 
Theorem 2.3. The free properad on an $\mathbb{S}$-bimodule $V$ is given by the sum on connected graphs (without level) $\mathcal{G}$ with the vertices indexed by elements of $V$

$$
\mathcal{F}(V)=\left(\bigoplus_{g \in \mathcal{G}_{c}} \bigotimes_{\nu \in \mathcal{N}} V(|\operatorname{Out}(\nu)|,|\operatorname{In}(\nu)|)\right) / \approx .
$$

The composition $\mu$ comes from the composition of directed graphs.

Remark. If $V$ is an $\mathbb{S}$-module, the connected graphs involved here are trees. Therefore, here we find the same construction of the free operad given in GK.

By the same arguments, we have that the free prop $\widetilde{\mathcal{F}}(V)$ on a saturated $\mathbb{S}$ bimodule $V$ is given by the sum on graphs (without level) indexed by elements of $V$. Here we find the same construction of the free prop as B. Enriquez and P. Etingof in EE].

Definition (Free prop). In this paper, we call a free prop on an $\mathbb{S}$-module $V$, and the image of $V$ by the following composition of left adjunct functors:

$$
\widetilde{\mathcal{F}}(V):=\mathcal{S} \circ \mathcal{F}(V) .
$$

Recall from [V1, the notion of split analytic functor.

Definition (Split analytic functors). A functor $f$ is a split analytic functor if it is a direct sum $\bigoplus_{n \in \mathbb{N}} f_{(n)}$ of homogenous polynomial functors of degree $n$.

One can decompose the set of directed (connected) graphs with the number of vertices. We denote by $\mathcal{G}_{c,(n)}$ the set of connected graphs with $n$ vertices. Hence, one has

$$
\begin{aligned}
\mathcal{F}(V) & =\left(\bigoplus_{g \in \mathcal{G}_{c}} \bigotimes_{\nu \in \mathcal{N}} V(|\operatorname{Out}(\nu)|,|\operatorname{In}(\nu)|)\right) / \approx \\
& =\bigoplus_{n \in \mathbb{N}} \underbrace{\left.\bigoplus_{g \in \mathcal{G}_{c,(n)}} \bigotimes_{i=1}^{n} V\left(\left|\operatorname{Out}\left(\nu_{i}\right)\right|,\left|\operatorname{In}\left(\nu_{i}\right)\right|\right)\right) / \approx}_{\mathcal{F}_{(n)}(V)} .
\end{aligned}
$$

Proposition 2.4. The functor $\mathcal{F}: V \mapsto \mathcal{F}(V)$ is a split analytic functor, where the part of weight $n$ is denoted as $\mathcal{F}_{(n)}(V)$. This graduation is stable under the composition $\mu$ of the free properad. Therefore, the free properad is a weight graded properad.

We define the counit $\epsilon$ by the following projection $\mathcal{F}(V) \rightarrow I=\mathcal{F}_{(0)}(V)$. With this augmentation map, the free properad is an augmented properad.

Lemma 2.5. Let $f=\bigoplus_{n=0}^{\infty} f_{(n)}$ be a split analytic functor in the category of $\mathbb{S}$ bimodules. Let $M=\bigoplus_{\rho \in \mathbb{N}} M^{(\rho)}$ be a weight graded $\mathbb{S}$-bimodule. The $\mathbb{S}$-bimodule $f(M)$ is bigraded with one graduation induced by the analytic functor and the other one given by the total weight. 
Proof. Denote $f_{(n)}=f_{n} \circ \Delta_{n}$ where $f_{n}$ is an $n$-linear morphism. The first graduation can be written $f(M)_{(n)}:=f_{n}(M, \ldots, M)$. The second one corresponds to

$$
f(M)^{(\rho)}:=\sum_{\substack{n \geq 1 \\ i_{1}+\cdots+i_{n}=\rho}} f_{n}\left(M^{\left(i_{1}\right)}, \ldots, M^{\left(i_{n}\right)}\right) .
$$

Corollary 2.6. Every free properad on a weight graded $\mathbb{S}$-bimodule is bigraded.

2.8. Cofree connected coproperad. We define the structure of a cofree connected coproperad on the same $\mathbb{S}$-bimodule as the free properad. Denote

$$
\mathcal{F}^{c}(V):=\mathcal{F}(V)=\left(\bigoplus_{g \in \mathcal{G}^{c}} \bigotimes_{\nu \in \mathcal{N}} V(|\operatorname{Out}(\nu)|,|\operatorname{In}(\nu)|)\right) / \approx .
$$

The projection on the summand generated by the trivial graph | gives the counit $\varepsilon: \mathcal{F}^{c}(V) \rightarrow I$.

The coproduct $\Delta$ lies on the set of cuttings of graphs into two parts. The image of an element $g(V)$ represented by a graph $g$ indexed by operations of $V$ under the coproduct $\Delta$ is given by the formula

$$
\Delta(g(V)):=\sum_{\left(g_{1}(V), g_{2}(V)\right)} g_{1}(V) \bigotimes_{c} g_{2}(V),
$$

where the sum is over pairs $\left(g_{1}(V), g_{2}(V)\right)$ of family of elements such that

$$
\mu\left(g_{1}(V) \bigotimes_{c} g_{2}(V)\right)=g(V) .
$$

Proposition 2.7. For every $\mathbb{S}$-bimodule $V,\left(\mathcal{F}^{c}(V), \Delta, \varepsilon\right)$ is a weight graded connected coproperad. This coproperad is cofree in the category of connected coproperads.

Proof. The counit relation comes from

$$
\begin{aligned}
\left(\varepsilon \bigotimes_{c} i d\right) \circ \Delta(g(V)) & =\left(\varepsilon \bigotimes_{c} i d\right) \circ \Delta\left(\sum_{\left(g_{1}(V), g_{2}(V)\right)} g_{1}(V) \bigotimes_{c} g_{2}(V)\right) \\
& =\sum_{\left(g_{1}(V), g_{2}(V)\right)} \varepsilon\left(g_{1}(V)\right) \bigotimes_{c} g_{2}(V) \\
& =I \bigotimes_{c} g(V) \\
& =g(V) .
\end{aligned}
$$

The coassociativity relation comes from

$$
\begin{aligned}
& \left(\Delta \bigotimes_{c} i d\right) \circ \Delta(g(V))=\left(i d \bigotimes_{c} \Delta\right) \circ \Delta(g(V)) \\
& =\sum_{\left(g_{1}(V), g_{2}(V), g_{3}(V)\right)} g_{1}(V) \bigotimes_{c} g_{2}(V) \bigotimes_{c} g_{3}(V),
\end{aligned}
$$

where the sum is over the triples $\left(g_{1}(V), g_{2}(V), g_{3}(V)\right)$ such that

$$
\mu\left(g_{1}(V) \otimes_{c} g_{2}(V) \otimes_{c} g_{3}(V)\right)=g(V) .
$$

Let $C$ be a connected coproperad and let $f: C \rightarrow V$ be a morphism of $\mathbb{S}$ bimodules. The analytic decomposition of $\mathcal{F}^{c}(V)$, according to the number of vertices of graphs, and the fact that $C$ is connected allows us to define by induction a 
morphism of connected coproperads $\bar{f}: C \rightarrow \mathcal{F}^{c}(V)$. This morphism is determined by $f$ and is the unique morphism of connected coproperads such that the following diagram commutes:

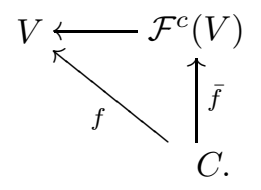

Remark. This construction generalizes the construction given by T. Fox in [F] for cofree connected coalgebras.

2.9. Quadratic properads and quadratic props. We define the notions of quadratic properad and quadratic prop. We relate these two notions and give examples.

In this paper, we are interested in the study of properads and props defined by generators and relations. Let $V$ be an $\mathbb{S}$-bimodule and $R$ be a sub-S-bimodule of $\mathcal{F}(V)$. Consider the ideal of $\mathcal{F}(V)$ generated by $R$. (For the notion of ideal in a monoidal category, we refer the reader to [V2].) The quotient properad $\mathcal{F}(V) /(R)$ is generated by $V$ and the relations $R$.

The Koszul duality for props deals with a subclass of properads and props composed by quadratic properads and quadratic props.

Definition (Quadratic properads). A quadratic properad is a properad defined by generators $V$ and relations $R$ such that $R$ is a sub-S-bimodule of $\mathcal{F}_{(2)}(V)$ (the part of weight 2 of $\mathcal{F}(V)$ ).

It means that $R$ is composed by linear combinations of connected graphs indexed by 2 elements of $V$.

Proposition 2.8. Every properad $\mathcal{P}=\mathcal{F}(V) /(R)$ defined by generators $V$ and relations $R$ such that $R$ is a sub-S-bimodule of $\mathcal{F}_{(n)}(V)$ is a weight graded properad.

Proof. Since the ideal $(R)$ is generated by a homogenous weight graded $\mathbb{S}$-bimodule, the quotient properad $\mathcal{F}(V) /(R)$ is weight graded. The elements of weight $n$ of $\mathcal{P}$ correspond to the image of $\mathcal{F}_{(n)}(V)$ under the projection $\mathcal{F}(V) \rightarrow \mathcal{P}=\mathcal{F}(V) /(R)$.

Corollary 2.9. Every quadratic properad is weight graded.

Once again, the same results hold for props.

Definition (Quadratic props). A quadratic prop is a prop defined by a generating $\mathbb{S}$-bimodule $V$ and relations $R$ such that $R$ is a sub-S-bimodule of $\widetilde{\mathcal{F}}_{(2)}(V)$ (the part of weight 2 of $\widetilde{\mathcal{F}}(V))$.

Quadratic properads and quadratic props are related by the following proposition.

Proposition 2.10. Let $V$ be an $\mathbb{S}$-bimodule and $R$ a sub-S-bimodule of $\mathcal{F}_{(2)}(V) \subset$ $\widetilde{\mathcal{F}}_{(2)}(V)$. The quotient prop $\widetilde{\mathcal{F}}(V) /(R)$ is isomorphic to $\mathcal{S}(\mathcal{F}(V) /(R))$, where $\mathcal{F}(V) /(R)$ is the quadratic properad generated by $V$ and $R$. 
Proof. Consider the morphism of props

$$
\widetilde{\mathcal{F}}(V)=\tilde{\mathcal{F}} \circ \mathcal{S}(V)=\mathcal{S} \circ \mathcal{F}(V) \stackrel{\Phi}{\longrightarrow} \mathcal{S}(\mathcal{F}(V) /(R)) .
$$

Since its kernel is the ideal generated by $R$ in $\mathcal{F}(V), \Phi$ induces an isomorphism $\widetilde{\mathcal{F}}(V) /(R) \stackrel{\bar{\Phi}}{\longrightarrow} \mathcal{S}(\mathcal{F}(V) /(R))$.

Remark. This proposition implies that, when the relations of a quadratic prop are defined by linear combinations of connected graphs, it is enough to study the related quadratic properad. In this case, the quadratic prop is obtained by concatenation from the quadratic properad. Since the concatenation product is well known in homology, it is enough to study the properad associated to some algebraic structures (gebras) to understand their homological properties (deformation, gebra up to homotopy) ( $c f$. 7.4).

Examples. Once again, the first examples come from the full subcategories $k$ Mod and $\mathbb{S}$-Mod. Quadratic algebras, like $S(V)$ and $\Lambda(V)$ (the original examples of J. - L. Koszul), are quadratic properads. Also, quadratic operads, like $\mathcal{A} s, \mathcal{C}$ om and $\mathcal{L}$ ie (coding associative, commutative and Lie algebras), are quadratic properads.

Examples. Let us give new examples treated by this theory. All the following properads are quadratic:

- The properad $\mathcal{B} i \mathcal{L}$ ie coding Lie bialgebras. This properad is generated by the following $\mathbb{S}$-bimodule:

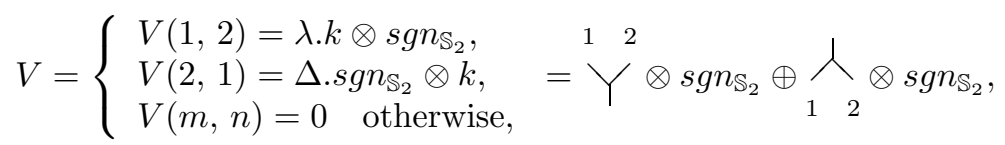

where $s g n_{\mathbb{S}_{2}}$ is the signature representation of $\mathbb{S}_{2}$. Its quadratic relations are given by

$$
\begin{aligned}
& R=\left\{\begin{array}{l}
\lambda(\lambda, 1)((123)+(231)+(312)) \\
\oplus((123)+(231)+(312))(\Delta, 1) \Delta \\
\oplus \Delta \otimes \lambda-(\lambda, 1) \otimes(213) \otimes(1, \Delta) \\
\quad-(1, \lambda)(\Delta, 1)-(1, \lambda)(\Delta, 1)-(1, \lambda) \otimes(132) \otimes(\Delta, 1)
\end{array}\right.
\end{aligned}
$$

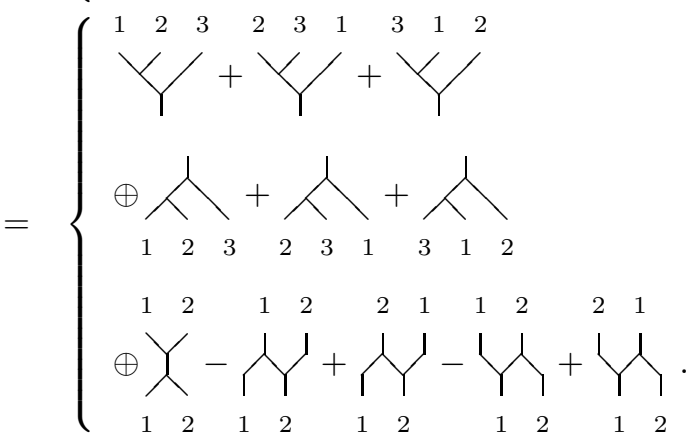

The $\mathcal{B}$ i $i$ e-gebras are exactly the Lie bialgebras introduced by V. Drinfeld ( $c f$. [Dr1]).

- One variation of Lie bialgebras has been introduced by M. Chas (cf. Chas.) in the framework of String Topology. An involutive Lie bialgebra is a Lie bialgebra such that $\lambda \circ \Delta=0$. Therefore, the associated properad is the 
same than $\mathcal{B} i \mathcal{L} i e$, where the "loop" $\$$ is added to the space of relations. We denote it by $\mathcal{B} i \mathcal{L} i e^{0}$, since every composition based on level graphs of genus $>0$ is null.

- The properad $\varepsilon \mathcal{B} i$ coding infinitesimal Hopf algebras. This properad is generated by the $\mathbb{S}$-bimodule

$$
V=\left\{\begin{array}{l}
V(1,2)=m . k \otimes k\left[\mathbb{S}_{2}\right] \\
V(2,1)=\Delta . k\left[\mathbb{S}_{2}\right] \otimes k \\
V(m, n)=0 \quad \text { otherwise. }
\end{array}=Y \oplus \lambda\right.
$$

Its relations are given by

$$
\begin{aligned}
R & =\left\{\begin{array}{l}
m(m, 1)-m(1, m) \\
\oplus(\Delta, 1) \Delta-(1, \Delta) \Delta \\
\oplus \Delta \otimes m-(m, 1)(1, \Delta)-(1, m)(\Delta, 1)
\end{array}\right. \\
& =\left\{\begin{array}{l}
\oplus \\
\oplus Y
\end{array}\right.
\end{aligned}
$$

These $\varepsilon \mathcal{B} i$-gebras are associative and non-commutative analogs of Lie bialgebras. They correspond to the infinitesimal Hopf algebras defined by M. Aguiar ( $c f$. Ag1, Ag2 and Ag3).

- The properad $\frac{1}{2} \mathcal{B} i$, the degenerated analogue of the properad of bialgebras. The generating $\mathbb{S}$-bimodule $V$ is the same as in the case of $\varepsilon \mathcal{B} i$, composed by a product and a coproduct. The relations $R$ are the following ones:

$$
\begin{aligned}
& R=\left\{\begin{array}{l}
m(m, 1)-m(1, m) \\
\oplus(\Delta, 1) \Delta-(1, \Delta) \Delta \\
\oplus \Delta \otimes m
\end{array}\right. \\
& =\left\{\begin{array}{l}
Y-Y \\
\oplus \lambda-\lambda \\
\oplus Y
\end{array}\right.
\end{aligned}
$$

This example has been introduced by M. Markl in Ma2 to describe a minimal model for the prop of bialgebras.

\section{Counterexamples.}

- The previous properad is a quadratic version of the properad $\mathcal{B} i$ coding bialgebras. This last one is defined by generators and relations but is not quadratic. The definition of $\mathcal{B} i$ is the same as $\frac{1}{2} \mathcal{B} i$ where the relation $(\kappa)$ 
is replaced by the following one:

$$
\begin{aligned}
\left(\kappa^{\prime}\right) & : \Delta \otimes m-(m, m) \otimes(1324) \otimes(\Delta, \Delta) \\
& =Y-Y_{Y} .
\end{aligned}
$$

Since the relation $\left(\kappa^{\prime}\right)$ includes a composition involving 4 generating operations, the properad $\mathcal{B} i$ is neither quadratic nor weight graded.

- Consider the prop coding unitary infinitesimal Hopf algebras defined by J.-L. Loday in [L4]. Its definition is the same as the prop $\varepsilon \mathcal{B} i$ where the third relation is

$$
\Delta \otimes m-(m, 1)(1, \Delta)-(1, m)(\Delta, 1)-(1,1)=X-\gamma-Y-\| .
$$

Since this relation is neither connected nor homogenous of weight 2 , one has that this prop is not quadratic and it does not come from a properad.

\section{Differential graded FrameWork}

In this section, we generalize the previous notions in the differential graded framework. For instance, we define the compositions products of a differential graded $\mathbb{S}$-bimodule. We give the definitions of quasi-free $\mathcal{P}$-modules and quasi-free properads.

3.1. The category of differential graded $\mathbb{S}$-bimodules. We now work in the category dg-Mod of differential graded $k$-modules. Recall that this category can be endowed with a tensor product $V \otimes_{k} W$ defined by the following formula:

$$
\left(V \otimes_{k} W\right)_{d}:=\bigoplus_{i+j=d} V_{i} \otimes_{k} W_{j}
$$

where the boundary map on $V \otimes_{k} W$ is given by

$$
\delta(v \otimes w):=\delta(v) \otimes w+(-1)^{|v|} v \otimes \delta(w),
$$

for $v \otimes w$ an elementary tensor and where $|v|$ denotes the homological degree of $v$. The symmetry isomorphisms involve Koszul-Quillen sign rules

$$
\tau_{v, w}: v \otimes w \mapsto(-1)^{|v||w|} w \otimes v .
$$

More generally, the commutation of two elements (morphisms, differentials, objects, etc.) of degree $d$ and $e$ induces a sign $(-1)^{d e}$.

Definition (dg-S-bimodule). A $d g$-S $\mathbb{S}$-bimodule $\mathcal{P}$ is a collection $(\mathcal{P}(m, n))_{m, n \in \mathbb{N}}$ of differential graded modules with an action of the symmetric group $\mathbb{S}_{m}$ on the left and an action of $\mathbb{S}_{n}$ on the right. These groups acts by morphisms of dg-modules. We denote by $\mathcal{P}_{d}(m, n)$ the sub- $\left(\mathbb{S}_{m}, \mathbb{S}_{n}\right)$-module composed by elements of degree $d$ of the chain complex $\mathcal{P}(m, n)$. 
3.2. Composition products of dg-S-bimodules. The composition products $\otimes_{c}$ and $\boldsymbol{\nabla}$ of $\mathbb{S}$-bimodules can be generalized to the differential graded framework. The definition remains the same except that the equivalence relation $\sim$ is based on symmetry isomorphisms and therefore involves Koszul-Quillen sign rules. For instance, one has

$$
\begin{array}{r}
\theta \otimes q_{1} \otimes \cdots \otimes q_{i} \otimes q_{i+1} \otimes \cdots \otimes q_{b} \otimes \sigma \otimes p_{1} \otimes \cdots \otimes p_{a} \otimes \omega \\
\sim(-1)^{\left|q_{i}\right|\left|q_{i+1}\right|} \theta^{\prime} \otimes q_{1} \otimes \cdots \otimes q_{i+1} \otimes q_{i} \otimes \cdots \otimes q_{b} \otimes \sigma^{\prime} \otimes p_{1} \otimes \cdots \otimes p_{a} \otimes \omega,
\end{array}
$$

where $\sigma^{\prime}=(1 \ldots i+1 i \ldots a)_{\bar{k}} \sigma$ and $\theta^{\prime}=\theta(1 \ldots i+1 i \ldots a)_{\bar{l}}^{-1}$.

Remark. To lighten the notations, we will often omit the induced representations $\theta$ and $\omega$ in the following text.

We define the image of an element $q_{1} \otimes \cdots \otimes q_{b} \otimes \sigma \otimes p_{1} \otimes \cdots \otimes p_{a}$ of $\mathcal{Q}(\bar{l}, \bar{k}) \otimes_{\mathbb{S}_{\bar{k}}}$ $k\left[\mathbb{S}_{\bar{k}, \bar{\jmath}}^{c}\right] \otimes_{\mathbb{S}_{\bar{l}}} \mathcal{P}(\bar{\jmath}, \bar{\imath})$ under the boundary map $\delta$ by the following formula:

$$
\begin{aligned}
& \delta\left(q_{1} \otimes \cdots \otimes q_{b} \otimes \sigma \otimes p_{1} \otimes \cdots \otimes p_{a}\right) \\
& =\sum_{\beta=1}^{b}(-1)^{\left|q_{1}\right|+\cdots+\left|q_{\beta-1}\right|} q_{1} \otimes \cdots \otimes \delta\left(q_{\beta}\right) \otimes \cdots \otimes q_{b} \otimes \sigma \otimes p_{1} \otimes \cdots \otimes p_{a} \\
& +\sum_{\alpha=1}^{a}(-1)^{\left|q_{1}\right|+\cdots+\left|q_{b}\right|+\left|p_{1}\right|+\cdots+\left|p_{\alpha-1}\right|} q_{1} \otimes \cdots \otimes q_{b} \otimes \sigma \otimes p_{1} \otimes \cdots \otimes \delta\left(p_{\alpha}\right) \otimes \cdots \otimes p_{a} .
\end{aligned}
$$

Lemma 3.1. The differential $\delta$ is constant on equivalence classes under the relation $\sim$.

Proof. The proof is straightforward and left to the reader.

One can also generalize the concatenation product $\otimes$ to the category of $\mathrm{dg}-\mathbb{S}$ bimodules.

Definition (Saturated differential graded $\mathbb{S}$-bimodule). A saturated differential graded $\mathbb{S}$-bimodule is a dg- $\mathbb{S}$-bimodule $\mathcal{P}$ such that $\mathcal{S}(\mathcal{P}) \cong \mathcal{P}$. We denote this category sat-dg-S-biMod.

One can extend Proposition 1.9 and Proposition 1.10 in the differential graded framework.

Proposition 3.2. Let $\mathcal{Q}$ and $\mathcal{P}$ be two differential graded $\mathbb{S}$-bimodules. Their connected composition product $\mathcal{Q} \bigotimes_{c} \mathcal{P}(m, n)$ is isomorphic to the $d g$-S-bimodule

$$
\bigoplus_{\Theta} k\left[\mathbb{S}_{m} / \mathbb{S}_{\bar{l}}^{B}\right] \otimes_{\mathbb{S}_{\bar{l}}} \mathcal{Q}(\bar{l}, \bar{k}) \otimes_{\mathbb{S}_{\bar{k}}} k\left[\mathbb{S}_{\bar{k}, \bar{\jmath}}^{c}\right] \otimes_{\mathbb{S}_{\bar{\jmath}}} \mathcal{P}(\bar{\jmath}, \bar{\imath}) \otimes_{\mathbb{s}_{\bar{\imath}}} k\left[\mathbb{S}_{\bar{\imath}}^{B} \backslash \mathbb{S}_{n}\right]
$$

and to the $d g$-S-bimodule

$$
\begin{gathered}
\bigoplus_{\Theta^{\prime}}\left(\left(\Pi_{1}^{\prime}\right) \mathcal{Q}\left(\left|\Pi_{1}^{\prime}\right|, k_{1}\right) \otimes_{k} \cdots \otimes_{k}\left(\Pi_{b}^{\prime}\right) \mathcal{Q}\left(\left|\Pi_{b}^{\prime}\right|, k_{b}\right)\right) \otimes_{\mathbb{S}_{\bar{k}}} k\left[\mathbb{S}_{\bar{k}, \bar{\jmath}}^{c}\right] \\
\otimes_{\mathbb{S}_{\bar{\jmath}}}\left(\mathcal{P}\left(j_{1},\left|\Pi_{1}\right|\right)\left(\Pi_{1}\right) \otimes_{k} \cdots \otimes_{k} \mathcal{P}\left(j_{a},\left|\Pi_{a}\right|\right)\left(\Pi_{a}\right)\right) .
\end{gathered}
$$

Once again, we have the same propositions for the composition product $\otimes$. We are going to study the homological properties of the composition product of two dg-S-bimodules. 
Remark that the chain complex $\left(\mathcal{Q} \otimes_{c} \mathcal{P}, \delta\right)$ corresponds to the total complex of a bicomplex. We define the bidegree of an element

$$
\begin{aligned}
\left(q_{1}, \ldots, q_{b}\right) \sigma\left(p_{1}, \ldots, p_{a}\right) \quad \text { of } \quad k\left[\mathbb{S}_{m} / \mathbb{S}_{\bar{l}}^{B}\right] & \otimes_{\mathbb{S}_{\bar{l}}} \mathcal{Q}(\bar{l}, \bar{k}) \\
& \otimes_{\mathbb{S}_{\bar{k}}} k\left[\mathbb{S}_{\bar{k}, \bar{\jmath}}^{c}\right] \otimes_{\mathbb{S}_{\bar{\jmath}}} \mathcal{P}(\bar{\jmath}, \bar{\imath}) \otimes_{\mathbb{S}_{\bar{\imath}}} k\left[\mathbb{S}_{\bar{\imath}}^{B} \backslash \mathbb{S}_{n}\right]
\end{aligned}
$$

by $\left(\left|q_{1}\right|+\cdots+\left|q_{b}\right|,\left|p_{1}\right|+\cdots+\left|p_{a}\right|\right)$. The horizontal differential $\delta_{h}: \mathcal{Q} \bigotimes_{c} \mathcal{P} \rightarrow \mathcal{Q} \bigotimes_{c} \mathcal{P}$ is induced by the one of $\mathcal{Q}$ and the vertical differential $\delta_{v}: \mathcal{Q} \otimes_{c} \mathcal{P} \rightarrow \mathcal{Q} \otimes_{c} \mathcal{P}$ is induced by the one of $\mathcal{P}$. One has explicitly,

$$
\begin{array}{ll} 
& \delta_{h}\left(\left(q_{1}, \ldots, q_{b}\right) \sigma\left(p_{1}, \ldots, p_{a}\right)\right) \\
& =\sum_{\beta=1}^{b}(-1)^{\left|q_{1}\right|+\cdots+\left|q_{\beta-1}\right|}\left(q_{1}, \ldots, \delta\left(q_{\beta}\right), \ldots, q_{b}\right) \sigma\left(p_{1}, \ldots, p_{a}\right) \\
\text { and } \quad & \delta_{v}\left(\left(q_{1}, \ldots, q_{b}\right) \sigma\left(p_{1}, \ldots, p_{a}\right)\right) \\
& =\sum_{\alpha=1}^{a}(-1)^{\left|q_{1}\right|+\cdots+\left|q_{b}\right|+\left|p_{1}\right|+\cdots+\left|p_{\alpha-1}\right|}\left(q_{1}, \ldots, q_{b}\right) \sigma\left(p_{1}, \ldots, \delta\left(p_{\alpha}\right), \ldots, p_{a}\right) .
\end{array}
$$

One can see that $\delta=\delta_{h}+\delta_{v}$ and that $\delta_{h} \circ \delta_{v}=-\delta_{v} \circ \delta_{h}$.

Like every bicomplex, this one gives rise to two spectral sequences $I^{r}(\mathcal{Q} \otimes \mathcal{P})$ and $I I^{r}(\mathcal{Q} \otimes \mathcal{P})$ that converge to the total homology $H_{*}(\mathcal{Q} \otimes \mathcal{P}, \delta)$. Recall that these two spectral sequences verify

$$
\begin{array}{lll}
I^{1}\left(\mathcal{Q} \bigotimes_{c} \mathcal{P}\right)=H_{*}\left(\mathcal{Q} \bigotimes_{c} \mathcal{P}, \delta\right), & I^{2}\left(\mathcal{Q} \bigotimes_{c} \mathcal{P}\right)=H_{*}\left(H_{*}\left(\mathcal{Q} \bigotimes_{c} \mathcal{P}, \delta_{v}\right), \delta_{h}\right) \\
\text { and } & I I^{1}\left(\mathcal{Q} \bigotimes_{c} \mathcal{P}\right)=H_{*}\left(\mathcal{Q} \bigotimes_{c} \mathcal{P}, \delta_{h}\right), & I I^{2}\left(\mathcal{Q} \bigotimes_{c} \mathcal{P}\right)=H_{*}\left(H_{*}\left(\mathcal{Q} \bigotimes_{c} \mathcal{P}, \delta_{h}\right), \delta_{v}\right) .
\end{array}
$$

Proposition 3.2 shows that the composition product $\mathcal{Q} \otimes_{c} \mathcal{P}$ can be decomposed as the direct sum of chain complexes

$$
\bigoplus_{\Theta} k\left[\mathbb{S}_{m} / \mathbb{S}_{\bar{l}}^{B}\right] \otimes_{\mathbb{S}_{\bar{l}}} \mathcal{Q}(\bar{l}, \bar{k}) \otimes_{\mathbb{S}_{\bar{k}}} k\left[\mathbb{S}_{\bar{k}, \bar{\jmath}}^{c}\right] \otimes_{\mathbb{S}_{\bar{\jmath}}} \mathcal{P}(\bar{\jmath}, \bar{\imath}) \otimes_{\mathbb{s}_{\bar{\imath}}} k\left[\mathbb{S}_{\bar{\imath}}^{B} \backslash \mathbb{S}_{n}\right]
$$

This decomposition is compatible with the structure of bicomplex. Therefore, the spectral sequences can be decomposed in the same way

$$
\begin{aligned}
I^{r}\left(\mathcal{Q} \otimes_{c} \mathcal{P}\right)=\bigoplus_{\Theta} I^{r}\left(k\left[\mathbb{S}_{m} / \mathbb{S}_{\bar{l}}^{B}\right] \otimes_{\mathbb{S}_{\bar{l}}} \mathcal{Q}(\bar{l}, \bar{k})\right. \\
\left.\otimes_{\mathbb{S}_{\bar{k}}} k\left[\mathbb{S}_{\bar{k}, \bar{\jmath}}^{c}\right] \otimes_{\mathbb{S}_{\bar{\jmath}}} \mathcal{P}(\bar{\jmath}, \bar{\imath}) k\left[\mathbb{S}_{\bar{\imath}}^{B} \backslash \mathbb{S}_{n}\right]\right) \\
\text { and } I I^{r}\left(\mathcal{Q} \otimes_{c} \mathcal{P}\right)=\bigoplus_{\Theta} I I^{r}\left(k\left[\mathbb{S}_{m} / \mathbb{S}_{\bar{l}}^{B}\right] \otimes_{\mathbb{S}_{\bar{\imath}}} \mathcal{Q}(\bar{l}, \bar{k}) \otimes_{\mathbb{S}_{\bar{k}}} k\left[\mathbb{S}_{\bar{k}, \bar{\jmath}}^{c}\right]\right. \\
\left.\otimes_{\mathbb{S}_{\bar{\jmath}}} \mathcal{P}(\bar{\jmath}, \bar{\imath}) \otimes_{\mathbb{S}_{\bar{\imath}}} k\left[\mathbb{S}_{\bar{\imath}}^{B} \backslash \mathbb{S}_{n}\right]\right)
\end{aligned}
$$

From these two formulas, one has the following proposition. 
Proposition 3.3. When $k$ is a field of characteristic 0 , one has

$$
\begin{aligned}
& I^{1}\left(k\left[\mathbb{S}_{m} / \mathbb{S}_{\bar{l}}^{B}\right] \otimes_{\mathbb{S}_{\bar{l}}} \mathcal{Q}(\bar{l}, \bar{k}) \otimes_{\mathbb{S}_{\bar{k}}} k\left[\mathbb{S}_{\bar{k}, \bar{\jmath}}^{c}\right] \otimes_{\mathbb{S}_{\bar{\jmath}}} \mathcal{P}(\bar{\jmath}, \bar{\imath}) \otimes_{\mathbb{S}_{\bar{\imath}}} k\left[\mathbb{S}_{\bar{\imath}}^{B} \backslash \mathbb{S}_{n}\right]\right) \\
= & k\left[\mathbb{S}_{m} / \mathbb{S}_{\bar{l}}^{B}\right] \otimes_{\mathbb{S}_{\bar{l}}} \mathcal{Q}(\bar{l}, \bar{k}) \otimes_{\mathbb{S}_{\bar{k}}} k\left[\mathbb{S}_{\bar{k}, \bar{\jmath}}^{c}\right] \otimes_{\mathbb{S}_{\bar{\jmath}}} H_{*}(\mathcal{P}(\bar{\jmath}, \bar{\imath})) \otimes_{\mathbb{S}_{\bar{\imath}}} k\left[\mathbb{S}_{\bar{\imath}}^{B} \backslash \mathbb{S}_{n}\right] \text { and } \\
& I I^{1}\left(k\left[\mathbb{S}_{m} / \mathbb{S}_{\bar{l}}^{B}\right] \otimes_{\mathbb{S}_{\bar{l}}} \mathcal{Q}(\bar{l}, \bar{k}) \otimes_{\mathbb{S}_{\bar{k}}} k\left[\mathbb{S}_{\bar{k}, \bar{\jmath}}^{c}\right] \otimes_{\mathbb{S}_{\bar{\jmath}}} \mathcal{P}(\bar{\jmath}, \bar{\imath}) \otimes_{\mathbb{S}_{\bar{\imath}}} k\left[\mathbb{S}_{\bar{\imath}}^{B} \backslash \mathbb{S}_{n}\right]\right) \\
= & k\left[\mathbb{S}_{m} / \mathbb{S}_{\bar{l}}^{B}\right] \otimes_{\mathbb{S}_{\bar{l}}} H_{*}(\mathcal{Q}(\bar{l}, \bar{k})) \otimes_{\mathbb{S}_{\bar{k}}} k\left[\mathbb{S}_{\bar{k}, \bar{\jmath}}^{c}\right] \otimes_{\mathbb{S}_{\bar{\jmath}}} \mathcal{P}(\bar{\jmath}, \bar{\imath}) \otimes_{\mathbb{S}_{\bar{\imath}}} k\left[\mathbb{S}_{\bar{\imath}}^{\left.B \backslash \mathbb{S}_{n}\right] .}\right.
\end{aligned}
$$

Proof. By Mashke's Theorem, we have that the rings of the form $k\left[\mathbb{S}_{\bar{\jmath}}\right]$ and $k\left[\mathbb{S}_{\bar{\imath}}\right]$ are semi-simple. Therefore, the modules $k\left[\mathbb{S}_{m} / \mathbb{S}_{\bar{l}}^{B}\right] \otimes_{\mathbb{S}_{\bar{l}}} \mathcal{Q}(\bar{l}, \bar{k}) \otimes_{\mathbb{S}_{\bar{k}}} k\left[\mathbb{S}_{\bar{k}, \bar{j}}^{c}\right]$ are $k\left[\mathbb{S}_{\bar{\jmath}}\right]$ projective modules (on the right) and the modules $k\left[\mathbb{S}_{\bar{\imath}}^{B} \backslash \mathbb{S}_{n}\right]$ are $k\left[\mathbb{S}_{\bar{\imath}}\right]$ projective modules (on the left) .

Proposition 3.4. When $k$ is a field of characteristic 0 , one has

$$
\begin{aligned}
& I^{2}\left(k\left[\mathbb{S}_{m} / \mathbb{S}_{\bar{l}}^{B}\right] \otimes_{\mathbb{S}_{\bar{l}}} \mathcal{Q}(\bar{l}, \bar{k}) \otimes_{\mathbb{S}_{\bar{k}}} k\left[\mathbb{S}_{\bar{k}, \bar{\jmath}}^{c}\right] \otimes_{\mathbb{S}_{\bar{\jmath}}} \mathcal{P}(\bar{\jmath}, \bar{\imath}) \otimes_{\mathbb{S}_{\bar{\imath}}} k\left[\mathbb{S}_{\bar{\imath}}^{B} \backslash \mathbb{S}_{n}\right]\right) \\
= & I I^{2}\left(k\left[\mathbb{S}_{m} / \mathbb{S}_{\bar{l}}^{B}\right] \otimes_{\mathbb{S}_{\bar{l}}} \mathcal{Q}(\bar{l}, \bar{k}) \otimes_{\mathbb{S}_{\bar{k}}} k\left[\mathbb{S}_{\bar{k}, \bar{\jmath}}^{c}\right] \otimes_{\mathbb{S}_{\bar{\jmath}}} \mathcal{P}(\bar{\jmath}, \bar{\imath}) \otimes_{\mathbb{S}_{\bar{\imath}}} k\left[\mathbb{S}_{\bar{\imath}}^{B} \backslash \mathbb{S}_{n}\right]\right) \\
= & k\left[\mathbb{S}_{m} / \mathbb{S}_{\bar{l}}^{B}\right] \otimes_{\mathbb{S}_{\bar{l}}} H_{*} \mathcal{Q}(\bar{l}, \bar{k}) \otimes_{\mathbb{S}_{\bar{k}}} k\left[\mathbb{S}_{\bar{k}, \bar{\jmath}}^{c}\right] \otimes_{\mathbb{S}_{\bar{\jmath}}} H_{*} \mathcal{P}(\bar{\jmath}, \bar{\imath}) \otimes_{\mathbb{S}_{\bar{\imath}}} k\left[\mathbb{S}_{\bar{\imath}}^{B} \backslash \mathbb{S}_{n}\right] .
\end{aligned}
$$

Proof. We use the same arguments to show that

$$
\begin{aligned}
& I^{2}\left(k\left[\mathbb{S}_{m} / \mathbb{S}_{\bar{l}}^{B}\right] \otimes_{\mathbb{S}_{\bar{l}}} \mathcal{Q}(\bar{l}, \bar{k}) \otimes_{\mathbb{S}_{\bar{k}}} k\left[\mathbb{S}_{\bar{k}, \bar{\jmath}}^{c}\right] \otimes_{\mathbb{S}_{\bar{\jmath}}} \mathcal{P}(\bar{\jmath}, \bar{\imath}) \otimes_{\mathbb{S}_{\bar{\imath}}} k\left[\mathbb{S}_{\bar{\imath}}^{B} \backslash \mathbb{S}_{n}\right]\right) \\
= & k\left[\mathbb{S}_{m} / \mathbb{S}_{\bar{l}}^{B}\right] \otimes_{\mathbb{S}_{\bar{l}}} H_{*}(\mathcal{Q}(\bar{l}, \bar{k})) \otimes_{\mathbb{S}_{\bar{k}}} k\left[\mathbb{S}_{\bar{k}, \bar{\jmath}}^{c}\right] \otimes_{\mathbb{S}_{\bar{\jmath}}} H_{*}(\mathcal{P}(\bar{\jmath}, \bar{\imath})) \otimes_{\mathbb{S}_{\bar{\imath}}} k\left[\mathbb{S}_{\bar{\imath}}^{B} \backslash \mathbb{S}_{n}\right] .
\end{aligned}
$$

We conclude by Künneth's formula

$$
\begin{aligned}
H_{*}(\mathcal{Q}(\bar{l}, \bar{k})) & =H_{*}\left(\mathcal{Q}\left(l_{1}, k_{1}\right) \otimes \cdots \otimes \mathcal{Q}\left(l_{a}, k_{a}\right)\right) \\
& \cong H_{*} \mathcal{Q}\left(l_{1}, k_{1}\right) \otimes \cdots \otimes H_{*} \mathcal{Q}\left(l_{a}, k_{a}\right)=H_{*} \mathcal{Q}(\bar{l}, \bar{k}) .
\end{aligned}
$$

Remark. Let $\Phi: M \rightarrow M^{\prime}$ and $\Psi: N \rightarrow N^{\prime}$ be two morphisms of dg-S-bimodules. The morphism $\Phi \bigotimes_{c} \Psi: M \bigotimes_{c} N \rightarrow M^{\prime} \bigotimes_{c} N^{\prime}$ is a morphism of bicomplexes. It induces morphisms of spectral sequences $I^{r}\left(\Phi \bigotimes_{c} \Psi\right): I^{r}\left(M \bigotimes_{c} N\right) \rightarrow I^{r}\left(M^{\prime} \bigotimes_{c} N^{\prime}\right)$ and $I I^{r}\left(\Phi \otimes_{c} \Psi\right): I I^{r}\left(M \otimes_{c} N\right) \rightarrow I I^{r}\left(M^{\prime} \otimes_{c} N^{\prime}\right)$.

More generally, we have the following proposition.

Proposition 3.5. When $k$ is a field of characteristic 0 , one has

$$
\begin{aligned}
& H_{*}\left(\mathcal{Q} \otimes_{c} \mathcal{P}\right)(m, n) \\
& \quad=\bigoplus_{\Theta} k\left[\mathbb{S}_{m} / \mathbb{S}_{\bar{l}}^{B}\right] \otimes_{\mathbb{S}_{\bar{\imath}}} H_{*} \mathcal{Q}(\bar{l}, \bar{k}) \otimes_{\mathbb{S}_{\bar{k}}} k\left[\mathbb{S}_{\bar{k}, \bar{\jmath}}^{c}\right] \otimes_{\mathbb{S}_{\bar{\jmath}}} H_{*} \mathcal{P}(\bar{\jmath}, \bar{\imath}) \otimes_{\mathbb{S}_{\bar{\imath}}} k\left[\mathbb{S}_{\bar{\imath}}^{B} \backslash \mathbb{S}_{n}\right] .
\end{aligned}
$$

That's to say, $H_{*}\left(\mathcal{Q} \bigotimes_{c} \mathcal{P}\right)=\left(H_{*} \mathcal{Q}\right) \bigotimes_{c}\left(H_{*} \mathcal{P}\right)$.

Proof. It is a direct corollary of Mashke's Theorem (for coinvariants) and Künneth's Theorem (for tensor products).

Once again, similar results apply to the connected composition products $\bigotimes$. 
Remark. These propositions generalize the results of B. Fresse for the composition product $\circ$ of operads to the composition products $\nabla_{c}$ and $\nabla$ of properads and props (cf. $\mathrm{Fr}$ 2.3).

3.3. Differential properads and differential props. We give here a differential variation of the notions of properads and props.

Definition (Differential properad). A differential properad $(\mathcal{P}, \mu, \eta)$ is a monoid in the monoidal category $\left(\mathrm{dg}\right.$-S-biMod, $\left.\mathbb{\Xi}_{c}, I\right)$ of differential graded $\mathbb{S}$-bimodules.

The composition morphism $\mu$ of a differential properad is a morphism of chain complexes of degree 0 compatible with the action of the symmetric groups $\mathbb{S}_{m}$ and $\mathbb{S}_{n}$. One has the derivation type relation:

$$
\begin{aligned}
& \delta\left(\mu\left(\left(p_{1}, \ldots, p_{b}\right) \sigma\left(p_{1}^{\prime}, \ldots, p_{a}^{\prime}\right)\right)\right) \\
& =\sum_{\beta=1}^{b}(-1)^{\left|p_{1}\right|+\cdots+\left|p_{\beta-1}\right|} \mu\left(\left(p_{1}, \ldots, \delta\left(p_{\beta}\right), \ldots, p_{b}\right) \sigma\left(p_{1}^{\prime}, \ldots, p_{a}^{\prime}\right)\right) \\
& +\sum_{\alpha=1}^{a}(-1)^{\left|p_{1}\right|+\cdots+\left|p_{b}\right|+\left|p_{1}^{\prime}\right|+\cdots+\left|p_{\alpha-1}^{\prime}\right|} \mu\left(\left(p_{1}, \ldots, p_{b}\right) \sigma\left(p_{1}^{\prime}, \ldots, \delta\left(p_{\alpha}^{\prime}\right), \ldots, p_{a}^{\prime}\right)\right) .
\end{aligned}
$$

Proposition 3.5 gives the following property.

Proposition 3.6. When $k$ is a field of characteristic 0, the homology groups $\left(H_{*}(\mathcal{P}), H_{*}(\mu), H_{*}(\eta)\right)$ of a differential properad $(\mathcal{P}, \mu, \eta)$ form a graded properad.

Definition (Differential weight graded $\mathbb{S}$-bimodule). A differential weight graded $\mathbb{S}$ bimodule $M$ is a direct sum over $\rho \in \mathbb{N}$ of differential $\mathbb{S}$-bimodules $M=\bigoplus_{\rho \in \mathbb{N}} M^{(\rho)}$.

The morphisms of differential weight graded $\mathbb{S}$-bimodules are the morphisms of differential $\mathbb{S}$-bimodules that preserve this decomposition. This forms a category denoted gr-dg-S-biMod.

Remark that the weight is a graduation separate from the homological degree that gives extra information.

Definition (Differential weight graded properad). A differential weight graded properad is a monoid in the monoidal category of differential weight graded $\mathbb{S}$ bimodules.

A differential weight graded properad $\mathcal{P}$ such that $\mathcal{P}^{(0)}=I$ is called connected.

Remark. Dually, we can define the notion of differential coproperad and weight graded differential coproperad. A differential coproperad is a coproperad where the coproduct is a morphism of $\mathrm{dg}$ - $\mathbb{S}$-bimodules verifying a coderivation type relation.

Similarly one has the notions of differential weight graded prop and coprop.

3.4. Free and quasi-free $\mathcal{P}$-modules. We define a variation of the notion of a free $\mathcal{P}$-module, namely the notion of quasi-free $\mathcal{P}$-module, and we prove some of its basic properties.

Let $\mathcal{P}$ be a (weight graded) dg-properad. Recall from 2.5, that the free differential $\mathcal{P}$-module on the right over a dg-S-bimodule $M$ is given by $L=M \bigotimes_{c} \mathcal{P}$, where the differential $\delta$ comes from the one of $M$ and the one of $\mathcal{P}$ as explained before. When $(\mathcal{P}, \mu, \eta, \epsilon)$ is an augmented dg-properad, one has that the indecomposable quotient of the free dg-P-P-module $L=M \otimes_{c} \mathcal{P}$ is isomorphic to $M$, as a dg- $\mathbb{S}$-bimodule. 
In the following part of the text, we will encounter differential $\mathcal{P}$-modules which are not strictly free. They are built on the $\mathrm{dg}$ - $\mathbb{S}$-bimodule $M \otimes_{c} \mathcal{P}$, but the boundary map is the sum of the canonical differential $\delta$ with an homogenous morphism of degree -1 .

Definition (Quasi-free $\mathcal{P}$-module). Let $\mathcal{P}$ be a differential properad. A (right) quasi-free $\mathcal{P}$-module $L$ is a dg-S-bimodule of the form $M \otimes_{c} \mathcal{P}$, where the differential $\delta_{\theta}$ is the sum $\delta+d_{\theta}$ of the canonical differential $\delta$ on the product $M \otimes_{c} \mathcal{P}$ with a homogenous morphism $d_{\theta}: M \bigotimes_{c} \mathcal{P} \rightarrow M \bigotimes_{c} \mathcal{P}$ of degree -1 such that

$$
\begin{aligned}
& d_{\theta}\left(\left(m_{1}, \ldots, m_{b}\right) \sigma\left(p_{1}, \ldots, p_{a}\right)\right) \\
& =\sum_{\beta=1}^{b}(-1)^{\left|m_{1}\right|+\cdots+\left|m_{\beta-1}\right|}\left(m_{1}, \ldots, d_{\theta}\left(m_{\beta}\right), \ldots, m_{b}\right) \sigma\left(p_{1}, \ldots, p_{a}\right) .
\end{aligned}
$$

In the weight graded framework, we ask $d_{\theta}$ to be a homogenous map for the weight such that

$$
d_{\theta}: M^{(\rho)} \rightarrow(\underbrace{M}_{(<\rho)} \bigotimes_{c} \underbrace{\mathcal{P}}_{(>1)})^{(\rho)}
$$

In this case, the boundary map $\delta_{\theta}$ preserves the global weight of $L$.

Therefore, the boundary map $\delta_{\theta}: M \otimes_{c} \mathcal{P} \rightarrow M \otimes_{c} \mathcal{P}$ verifies the following relation:

$$
\begin{aligned}
& \delta_{\theta}\left(\left(m_{1}, \ldots, m_{b}\right) \sigma\left(p_{1}, \ldots, p_{a}\right)\right) \\
& =\sum_{\beta=1}^{b}(-1)^{\left|m_{1}\right|+\cdots+\left|m_{\beta-1}\right|}\left(m_{1}, \ldots, \delta_{\theta}\left(m_{\beta}\right), \ldots, m_{b}\right) \sigma\left(p_{1}, \ldots, p_{a}\right) \\
& +\sum_{\alpha=1}^{a}(-1)^{\left|m_{1}\right|+\cdots+\left|m_{b}\right|+\left|p_{1}\right|+\cdots+\left|p_{\alpha-1}\right|}\left(m_{1}, \ldots, m_{b}\right) \sigma\left(p_{1}, \ldots, \delta\left(p_{\alpha}\right), \ldots, p_{a}\right) .
\end{aligned}
$$

Since $\delta^{2}=0$, the identity $\delta_{\theta}^{2}=0$ is equivalent to $\delta d_{\theta}+d_{\theta} \delta+d_{\theta}{ }^{2}=0$.

If one writes $d_{\theta}\left(m_{\beta}\right)=\left(m_{1}^{\prime}, \ldots, m_{b^{\prime}}^{\prime}\right) \sigma^{\prime}\left(p_{1}^{\prime}, \ldots, p_{a^{\prime}}^{\prime}\right) \in M \otimes_{c} \mathcal{P}$, then one has

$$
\begin{aligned}
& \left(m_{1}, \ldots, d_{\theta}\left(m_{\beta}\right), \ldots, m_{b}\right) \sigma\left(p_{1}, \ldots, p_{a}\right) \\
& \times\left(m_{1}, \ldots, m_{1}^{\prime}, \ldots, m_{b^{\prime}}^{\prime}, \ldots, m_{b}\right) \sigma^{\prime} \mu\left(\left(p_{1}^{\prime}, \ldots, p_{a^{\prime}}^{\prime}\right) \sigma^{\prime \prime}\left(p_{1}, \ldots, p_{a}\right)\right) \in M \otimes_{c} \mathcal{P} .
\end{aligned}
$$

Notice that the morphism $d_{\theta}$ is determined by its restriction on $M \stackrel{M \otimes_{c} \eta}{\longrightarrow} M \otimes_{c} \mathcal{P}$.

Proposition 3.7. The morphism $M \stackrel{M \bigotimes_{c} \eta}{\longrightarrow} M \bigotimes_{c} \mathcal{P}$ is a morphism of $d g$-S-bimodules if and only if $d_{\theta}=0$.

When $\mathcal{P}$ is an augmented dg-properad, the condition for the morphism $M \bigotimes_{c} \varepsilon$ to be a morphism of differential graded modules is weaker.

Proposition 3.8. Let $(\mathcal{P}, \mu, \eta, \varepsilon)$ be an augmented dg-properad. The morphism $M \bigotimes_{c} \mathcal{P} \stackrel{M \otimes_{c} \varepsilon}{\longrightarrow} M$ is a morphism of $d g$-S-bimodules if and only if the following composition is null:

$$
M \otimes_{c} \mathcal{P} \stackrel{d_{\theta}}{\longrightarrow} M \otimes_{c} \mathcal{P} \stackrel{M \otimes_{c} \varepsilon}{\longrightarrow} M=0
$$


Corollary 3.9. In this case, the indecomposable quotient of $L=M \bigotimes_{c} \mathcal{P}$ is isomorphic to $M$ as a $d g$-S-bimodule.

Definition (Morphism of weight graded quasi-free $\mathcal{P}$-module). Let $L=M \otimes_{c} \mathcal{P}$ and $L^{\prime}=M^{\prime} \otimes_{c} \mathcal{P}$ be two weight graded quasi-free $\mathcal{P}$-modules. A morphism $\Phi: L \rightarrow L^{\prime}$ of weight graded differential $\mathcal{P}$-modules is a morphism of weight graded quasi-free $\mathcal{P}$-modules if $\Phi$ preserves the graduation on the left

$$
\Phi: \underbrace{M}_{(\rho)} \otimes_{c} \mathcal{P} \rightarrow \underbrace{M^{\prime}}_{(\rho)} \otimes_{c} \mathcal{P} .
$$

In the following of the text, the examples of weight graded quasi-free $\mathcal{P}$-modules that we will have to treat will have a particular form.

Definition (Analytic quasi-free $\mathcal{P}$-module). Let $\mathcal{P}$ be a weight graded differential properad. An analytic quasi-free $\mathcal{P}$-module is a quasi-free $\mathcal{P}$-module $L=M \otimes_{c} \mathcal{P}$ such that the weight graded differential $\mathbb{S}$-bimodule $M$ is given by the image $M=$ $\Upsilon(V)$ of a weight graded differential $\mathbb{S}$-bimodule $V$ under a split analytic functor $\Upsilon$ (cf. 2.7). Denote $\Upsilon=\bigoplus_{n \in \mathbb{N}} \Upsilon_{(n)}$, where $\Upsilon_{(n)}$ is a homogenous polynomial functor of degree $n$ and such that $\Upsilon_{(0)}=I$. Moreover, $V$ must verify $V^{(0)}=0$ so that $\Upsilon(V)^{(0)}=I$. As we have seen in Lemma 2.5. $\Upsilon(V)$ is bigraded. The morphism $d_{\theta}$ is supposed to verify

$$
d_{\theta}: \Upsilon_{(n)}(V)^{(\rho)} \rightarrow \underbrace{\Upsilon(V)}_{n-1} \otimes_{c} \underbrace{\mathcal{P}}_{1}
$$

where the graduation $(\rho)$ is the global graduation coming from the one of $V$ and $(n)$ is the degree of the analytic functor $\Upsilon$.

Examples. The examples of analytic quasi-free $\mathcal{P}$-modules fall into two parts:

- When $V=\overline{\mathcal{P}}$, we have the example of the (differential) bar construction $\Upsilon(V)=\overline{\mathcal{B}}(\mathcal{P})=\mathcal{F}^{c}(\Sigma \overline{\mathcal{P}})(c f$. Section 4). The augmented bar construction $\overline{\mathcal{B}}(\mathcal{P}) \otimes_{c} \mathcal{P}$ is an analytic quasi-free $\mathcal{P}$-modules.

- When $\mathcal{P}$ is quadratic properad generated by a dg-S-bimodule $V$, the Koszul dual coproperad $\mathcal{P}^{\mathrm{i}}$ is a split analytic functor in $V$ ( $c f$. Section 7 ). Therefore, the Koszul complex $\mathcal{P} i \bigotimes_{c} \mathcal{P}$ is an analytic quasi-free $\mathcal{P}$-module.

We have immediately the following proposition.

Proposition 3.10. Let $\mathcal{P}$ be an augmented weight graded dg-properad and $L=$ $\Upsilon(V) \bigotimes_{c} \mathcal{P}$ be an analytic quasi-free $\mathcal{P}$-module. We have

$$
M \otimes_{c} \mathcal{P} \stackrel{d_{\theta}}{\longrightarrow} M \otimes_{c} \mathcal{P} \stackrel{M \otimes_{c} \varepsilon}{\longrightarrow} M=0 .
$$

Therefore, we can identify the indecomposable quotient of $L$ to $\Upsilon(V)$.

Remark. Dually, we have the notions of a differential cofree $\mathcal{C}$-comodule on a dgS-bimodule $M$, given by $M \otimes_{C} \mathcal{C}$, a quasi-cofree $\mathcal{C}$-comodule and an analytic quasicofree $\mathcal{C}$-comodule. The augmented cobar construction $\overline{\mathcal{B}}^{c}(\mathcal{C}) \otimes_{c} \mathcal{C}$ is an example of an analytic quasi-free $\mathcal{C}$-comodule. 
3.5. Free and quasi-free properads. Here we define the notion of a free differential properad. As in the case of $\mathcal{P}$-modules, we will have to deal with differential properads that are not strictly free. Therefore, we introduce the notion of a quasifree properad which is a free properad where the differential is given by the sum of the canonical one with a derivation.

Let $(V, \delta)$ be a dg-S-bimodule. We have seen in 2.7 that the free properad on $V$ is given by the weight graded $\mathbb{S}$-bimodule $\mathcal{F}(V)$ which is the direct sum on connected graphs (without level) where the vertices are indexed by elements of $V$,

$$
\mathcal{F}(V)=\left(\bigoplus_{g \in \mathcal{G}_{c}} \bigotimes_{\nu \in \mathcal{N}} V(|\operatorname{Out}(\nu)|,|\operatorname{In}(\nu)|)\right) / \approx .
$$

This construction corresponds to the quotient of the simplicial $\mathbb{S}$-bimodule $\mathcal{F} \mathcal{S}(V)=\bigoplus_{n \in \mathbb{N}}\left(V_{+}\right)^{\bigotimes_{c} n}$ under the relation generated by $I \bigotimes_{c} V \equiv V \bigotimes_{c} I$. In the differential graded framework, one has to take the signs into account. In this case, we quotient $\mathcal{F} \mathcal{S}(V)$ by the relation

$$
\left(\nu_{1}, \nu, \nu_{2}\right) \sigma\left(\nu_{1}^{\prime}, 1, \nu_{2}^{\prime}\right) \equiv(-1)^{\left|\nu_{2}\right|+\left|\nu_{1}^{\prime}\right|}\left(\nu_{1}, 1,1, \nu_{2}\right) \sigma^{\prime}\left(\nu_{1}^{\prime}, \nu, \nu_{2}^{\prime}\right),
$$

for instance, where $\nu$ belongs to $V(2,1)$.

Proposition 3.11. The canonical differential $\delta$ defined on $\mathcal{F} \mathcal{S}(V)$ by the one of $V$ is constant on the equivalence classes for the relation $\equiv$.

Proof. The verification of signs is straightforward and is the same as in Lemma 3.1 .

Theorem 3.12. The differential properad $(\mathcal{F}(V), \delta)$ is free on the $d g$-S-bimodule $(V, \delta)$.

Remark. Since the analytic decomposition of the functor $\mathcal{F}(V)$ given in Proposition 2.4 is stable under the differential $\delta$, we have that $\mathcal{F}(V)$ is a weight graded differential properad.

The projection $\mathcal{F}(V) \rightarrow I$ is a morphism of dg-properads.

The functor $\mathcal{F}$ shares interesting homological properties.

Proposition 3.13. Over a field $k$ of characteristic 0 , the functor $\mathcal{F}: d g$-S -biMod $\rightarrow$ dg-properad is exact. We have $H_{*}(\mathcal{F}(V))=\mathcal{F}\left(H_{*}(V)\right)$.

Proof. The proof of this proposition is the same as the proof of Theorem 4 of the article of M. Markl and A. A. Voronov [MV] and lies, once again, on Mashke's theorem.

Definition (Derivation). Let $\mathcal{P}$ be a dg-properad. A homogenous morphism of $\mathbb{S}$ bimodules $d: \mathcal{P} \rightarrow \mathcal{P}$ of (homological) degree $|d|$ is called a derivation if it satisfies the following identity:

$$
\begin{aligned}
& \delta\left(\mu\left(\left(p_{1}, \ldots, p_{b}\right) \sigma\left(p_{1}^{\prime}, \ldots, p_{a}^{\prime}\right)\right)\right) \\
& =\sum_{\beta=1}^{b}(-1)^{\left(\left|p_{1}\right|+\cdots+\left|p_{\beta-1}\right|\right)|d|} \mu\left(\left(p_{1}, \ldots, \delta\left(p_{\beta}\right), \ldots, p_{b}\right) \sigma\left(p_{1}^{\prime}, \ldots, p_{a}^{\prime}\right)\right) \\
& +\sum_{\alpha=1}^{a}(-1)^{\left(\left|p_{1}\right|+\cdots+\left|p_{b}\right|+\left|p_{1}^{\prime}\right|+\cdots+\left|p_{\alpha-1}^{\prime}\right|\right)|d|} \mu\left(\left(p_{1}, \ldots, p_{b}\right) \sigma\left(p_{1}^{\prime}, \ldots, \delta\left(p_{\alpha}^{\prime}\right), \ldots, p_{a}^{\prime}\right)\right) .
\end{aligned}
$$


For example, the differential $\delta$ of a differential properad is a derivation of degree -1 such that $\delta^{2}=0$. Notice that the sum $\delta+d$ of the differential $\delta$ with a derivation $d$ of degree -1 is a derivation of the same degree. In this case, since $\delta^{2}=0$ the equation $\left(\delta^{+} d\right)^{2}$ is equivalent to $\delta d+d \delta+d^{2}=0$.

Remark. Dually, one can define the notion of coderivation on a differential coprop$\operatorname{erad} \mathcal{C}$.

The next proposition gives the form of the derivations on the free differential properad $\mathcal{F}(V)$.

Proposition 3.14. Let $\mathcal{F}(V)$ be the free dg-properad on the $d g$-S-bimodule $V$. For any homogenous morphism $\theta: V \rightarrow \mathcal{F}(V)$, there exists a unique derivation $d_{\theta}: \mathcal{F}(V) \rightarrow \mathcal{F}(V)$ with the same degree such that its restriction to $V$ is equal to $\theta$. This correspondence is one-to-one.

Moreover, if $\theta: V \rightarrow \mathcal{F}_{(r)}(V) \subset \mathcal{F}(V)$, we have $d_{\theta}\left(\mathcal{F}_{(s)}(V)\right) \subset \mathcal{F}_{(r+s-1)}(V)$.

Proof. Let $g$ be a graph with $n$ vertices. We define $d_{\theta}$ on a representative of an equivalence class for the relation $\equiv$ (vertical move of a vertex up to sign) associated to the graph $g$. This corresponds to a choice of an order for writing the elements of $V$ that index the vertices of $g$. Write this representative $\bigotimes_{i=1}^{n} \nu_{i}$, with $\nu_{i} \in V$. Hence, we define $d_{\theta}$ by the following formula:

$$
d_{\theta}\left(\bigotimes_{i=1}^{n} \nu_{i}\right)=\sum_{i=1}^{n}(-1)^{\left|\nu_{1}\right|+\cdots+\left|\nu_{i-1}\right|}\left(\bigotimes_{j=1}^{i-1} \nu_{j}\right) \otimes \theta\left(\nu_{i}\right) \otimes\left(\bigotimes_{j=i+1}^{n} \nu_{j}\right)
$$

One can see that $d_{\theta}$ is well defined on the equivalence classes for the relation $\equiv$. Applying $d_{\theta}$ is given by applying $\theta$ to every element of $V$ indexing a vertex of $g$. (Since the application $\theta$ is an application of $\mathbb{S}$-bimodules, the morphism $d_{\theta}$ is well defined on the equivalence classes for the relation $\approx$.)

The product $\mu$ of the free properad $\mathcal{F}(V)$ is surjective. Hence, it shows that every derivation is of this form.

If the image under $\theta$ of the elements of $V$ is a sum of elements of $\mathcal{F}(V)$ that can be written by graphs with $r$ vertices ( $\theta$ creates $r-1$ vertices), the form of $d_{\theta}$ shows that $d_{\theta}\left(\mathcal{F}_{(s)}(V)\right) \subset \mathcal{F}_{(r+s-1)}(V)$.

Dually, we have the same proposition for the coderivations of the cofree connected coproperad.

Proposition 3.15. Let $\mathcal{F}^{c}(V)$ be the cofree connected dg-coproperad on the $d g-\mathbb{S}$ bimodule $V$. For any homogenous morphism $\theta: \mathcal{F}^{c}(V) \rightarrow V$, there exists a unique coderivation $d_{\theta}: \mathcal{F}^{c}(V) \rightarrow \mathcal{F}^{c}(V)$ such that the composition

$$
\mathcal{F}^{c}(V) \stackrel{d_{\theta}}{\longrightarrow} \mathcal{F}^{c}(V) \stackrel{\text { proj }}{\longrightarrow} V
$$

is equal to $\theta$. This correspondence is one-to-one.

Moreover, if $\theta: \mathcal{F}^{c}(V) \rightarrow V$ is null on the components $\mathcal{F}_{(r)}^{c}(V) \subset \mathcal{F}^{c}(V)$, for $s \neq r$, we have $d_{\theta}\left(\mathcal{F}_{(s+r-1)}(V)\right) \subset \mathcal{F}_{(s)}(V)$, for all $s>0$.

Graphically, applying the coderivation $d_{\theta}$ on an element of $\mathcal{F}^{c}(V)$ represented by a graph $g$ corresponds to applying $\theta$ on every admissible sub-graph of $g$.

We can now state the definition of a quasi-free properad. 
Definition. (Quasi-free properad) A quasi-free properad $\left(\mathcal{P}, \delta_{\theta}\right)$ is a dg-properad of the form $\mathcal{P}=\mathcal{F}(V)$, with $(V, \delta)$ a dg-S-bimodule and where the differential $\delta_{\theta}: \mathcal{F}(V) \rightarrow \mathcal{F}(V)$ is the sum of the canonical differential $\delta$ with a derivation $d_{\theta}$ of degree $-1, \delta_{\theta}=\delta+d_{\theta}$.

The previous proposition shows that every derivation $d_{\theta}$ on $\mathcal{F}(V)$ is determined by its restriction $\theta$ on $V$. Therefore, the inclusion $V \rightarrow \mathcal{F}(V)$ is a morphism of dg-S-bimodules if and only if $\theta$ is null. On the contrary, the projection $\mathcal{F}(V) \rightarrow V$ is a morphism of $\mathrm{dg}$ - $\mathbb{S}$-bimodules under a weaker condition.

Proposition 3.16. The projection $\mathcal{F}(V) \rightarrow V$ is a morphism of $d g$-S্S-bimodules if and only if $\theta(V) \subset \bigoplus_{r \geq 2} \mathcal{F}_{(r)}(V)$.

In this case, we say that the differential is decomposable.

Dually, we have the notion of a quasi-cofree coproperad.

Definition (Quasi-cofree coproperad). A quasi-cofree coproperad $\left(\mathcal{C}, \delta_{\theta}\right)$ is a dgcoproperad of the form $\mathcal{C}=\mathcal{F}^{c}(V)$, with $(V, \delta)$ a dg-S-bimodule and where the differential $\delta_{\theta}: \mathcal{F}^{c}(V) \rightarrow \mathcal{F}^{c}(V)$ is the sum of the canonical differential $\delta$ with a coderivation $d_{\theta}$ of degree $-1, \delta_{\theta}=\delta+d_{\theta}$.

The previous proposition on coderivations of $\mathcal{F}^{c}(V)$ shows that the projection $\mathcal{F}^{c}(V) \rightarrow V$ is a morphism of dg-S-bimodules if and only if $\theta$ is null and that the inclusion $V \rightarrow \mathcal{F}^{c}(V)$ is a morphism of dg-S-bimodules if and only if $\theta$ is null on $\mathcal{F}_{(1)}^{c}(V)=V$.

Examples. The fundamental examples of a quasi-free properad and a quasi-cofree coproperad are given by the cobar construction $\mathcal{F}\left(\Sigma^{-1} \overline{\mathcal{C}}\right)$ and the bar construction $\mathcal{F}^{c}(\Sigma \overline{\mathcal{P}})$. These two constructions are studied in the next section.

\section{BAR AND COBAR CONSTRUCTION}

We generalize the bar and cobar constructions of associative algebras ( $c f$. Séminaire H. Cartan [C] ) and for operads (cf. E. Getzler, J. Jones [GJ]) to properads. First, we study the properties of the partial composition products and coproducts. We define the reduced bar and cobar constructions and then the bar and cobar constructions with coefficients. These constructions share interesting homological properties. For instance, they induce resolutions for differential properads and props (cf. Theorem 7.8 and Section 7). To show these resolutions, we prove that the augmented bar and cobar constructions are acyclic, at the end of this section.

4.1. Partial composition product and coproduct. We define the notions of suspension and desuspension of a dg-S-bimodule. Then, we give the definitions and first properties of the partial composition products and coproducts.

4.1.1. Suspension and desuspension of a $\mathbf{d g}$ - $\mathbb{S}$-bimodule. Let $\Sigma$ be the graded S-bimodule defined by

$$
\begin{cases}\Sigma(0,0)=k . \Sigma & \text { where } \Sigma \text { is an element of degree }+1 \\ \Sigma(m, n)=0 & \text { otherewise. }\end{cases}
$$

Definition (Suspension $\Sigma V$ ). The suspension of the dg-S-bimodule $V$ is the dgS-bimodule $\Sigma V:=\Sigma \otimes V$. 
Taking the suspension of a dg-S-bimodule $V$ corresponds to taking the tensor product of every element of $V$ with $\Sigma$. To an element $v \in V_{d-1}$, one associates $s \otimes v \in(\Sigma V)_{d}$, which is often denoted $\Sigma v$. Therefore, $(\Sigma V)_{d}$ is naturally isomorphic to $V_{d-1}$. Following the same rules as in the previous section, the differential on $\Sigma V$ is given by the formula $\delta(\Sigma v)=-\Sigma \delta(v)$, for every $v$ in $V$. The suspension $\Sigma V$ corresponds to the introduction of an extra element of degree +1 . This involves Koszul-Quillen sign rules when permutating objects.

In the same way, we define the dg-S-bimodule $\Sigma^{-1}$ by

$$
\begin{cases}\Sigma^{-1}(0,0)=k \cdot \Sigma^{-1} & \text { where } \Sigma^{-1} \text { is an element of degree }-1, \\ \Sigma^{-1}(m, n)=0 & \text { otherwise. }\end{cases}
$$

Definition (Desuspension $\Sigma^{-1} V$ ). The desuspension of the dg-S-bimodule $V$ is the dg-S-bimodule $\Sigma^{-1} V:=\Sigma^{-1} \otimes V$.

4.1.2. Partial composition product. Let $(\mathcal{P}, \mu, \eta, \varepsilon)$ be an augmented properad. Since 2-level connected graphs are endowed with a bigraduation given by the number of vertices on each level, we can decompose the composition product $\mu$ on connected graphs in the following way: $\mu=\bigoplus_{r, s \in \mathbb{N}} \mu_{(r, s)}$, where

$$
\mu_{(r, s)}:(I \oplus \underbrace{\overline{\mathcal{P}}}_{r}) \bigotimes_{c}(I \oplus \underbrace{\overline{\mathcal{P}}}_{s}) \rightarrow \overline{\mathcal{P}} .
$$

The product $\mu_{(r, s)}$ gives the composition of $r$ non-trivial operations with $s$ nontrivial operations.

Definition (Partial composition product). We call a partial composition product the restriction $\mu_{(1,1)}$ of the composition product $\mu$ to $(I \oplus \underbrace{\overline{\mathcal{P}}}_{1}) \otimes_{c}(I \oplus \underbrace{\overline{\mathcal{P}}}_{1})$.

Notice that the partial composition product is the composition product of two non-trivial elements of $\mathcal{P}$ where at least one output of the first one is linked to one input of the second one.

Remark. When $\mathcal{P}$ is an operad, this partial composition product is exactly the partial product denoted $\circ_{i}$ by V. Ginzburg and M.M. Kapranov in GK. In the framework of $\frac{1}{2}$-PROPs introduced by M. Markl and A.A. Voronov in [MV], these authors only consider partial composition products where the upper element has only one output or where the lower element has only one input. The partial composition product defined by W.L. Gan in $[\mathrm{G}$ ] on dioperads only involves graphs where the two elements of $\overline{\mathcal{P}}$ are linked by a unique edge. In these cases, the related monoidal product is generated by the partial composition product (every composition can be written with a finite number of partial products). One can see that the composition product of a properad has the same property.

Lemma 4.1. Let $(\mathcal{P}, \mu, \eta, \varepsilon)$ be an augmented dg-properad. The partial composition product $\mu_{(1,1)}$ induces a homogenous morphism $\theta$ of degree -1 :

$$
\theta: \mathcal{F}_{(2)}^{c}(\Sigma \overline{\mathcal{P}})=(I \oplus \underbrace{\Sigma \overline{\mathcal{P}}}_{1}) \otimes_{c}(I \oplus \underbrace{\Sigma \overline{\mathcal{P}}}_{1}) \rightarrow \Sigma \overline{\mathcal{P}} .
$$

Recall that when $\mathcal{P}$ is a dg-S-bimodule, the free properad $\mathcal{F}(\Sigma \overline{\mathcal{P}})$ on $\Sigma \overline{\mathcal{P}}$ is bigraded. The first graduation comes from the number of operations of $\Sigma \overline{\mathcal{P}}$ used to represent an element of $\mathcal{F}(\Sigma \overline{\mathcal{P}})$. We denote this graduation $\mathcal{F}_{(n)}(\Sigma \overline{\mathcal{P}})$. The second 
graduation, the homological degree, is given by the sum of the homological degrees of the operations of $\Sigma \overline{\mathcal{P}}$.

Proof. Let $(1, \ldots, 1, \Sigma q, 1, \ldots, 1) \sigma(1, \ldots, 1, \Sigma p, 1, \ldots, 1)$ represent a homogenous element of homological degree $|q|+|p|+2$ of $\mathcal{F}_{(2)}^{c}(\Sigma \overline{\mathcal{P}})$. We define $\theta$ by the following formula:

$$
\begin{aligned}
& \theta((1, \ldots, 1, \Sigma q, 1, \ldots, 1) \sigma(1, \ldots, 1, \Sigma p, 1, \ldots, 1)) \\
& :=(-1)^{|q|} \Sigma \mu_{(1,1)}(1, \ldots, 1, q, 1, \ldots, 1) \sigma(1, \ldots, 1, p, 1, \ldots, 1) .
\end{aligned}
$$

Since $\mathcal{P}$ is a differential properad, this last element has degree $|q|+|p|+1$.

With Proposition 3.15, we can associate a coderivation $d_{\theta}: \mathcal{F}^{c}(\Sigma \overline{\mathcal{P}}) \rightarrow \mathcal{F}^{c}(\Sigma \overline{\mathcal{P}})$ to any homogenous morphism $\theta$.

Proposition 4.2. The coderivation $d_{\theta}$ has the following properties :

(1) The equation $\delta d_{\theta}+d_{\theta} \delta=0$ is true if and only if the partial composition product $\mu_{(1,1)}$ is a morphism of $d g$-S-bimodules.

(2) We have $d_{\theta}^{2}=0$.

Proof.

(1) Following Proposition [3.15, applying $d_{\theta}$ to an element of $\mathcal{F}^{c}(\Sigma \overline{\mathcal{P}})$ represented by a graph $g$ corresponds to applying $\theta$ to every possible sub-graph of $g$. Here, we apply $\theta$ to every couple of vertices linked by at least one edge and such that there exists no vertex between. For a couple of such vertices indexed by $\Sigma q$ and $\Sigma p$, we have

$$
\begin{aligned}
& \delta \circ d_{\theta}((1, \ldots, 1, \Sigma q, 1, \ldots, 1) \sigma(1, \ldots, 1, \Sigma p, 1, \ldots, 1)) \\
& =(-1)^{|q|} \delta\left(\Sigma \mu_{(1,1)}((1, \ldots, 1, q, 1, \ldots, 1) \sigma(1, \ldots, 1, p, 1, \ldots, 1))\right) \\
& =(-1)^{|q|+1} \Sigma \delta\left(\mu_{(1,1)}((1, \ldots, 1, q, 1, \ldots, 1) \sigma(1, \ldots, 1, p, 1, \ldots, 1))\right)
\end{aligned}
$$

and

$$
\begin{aligned}
& d_{\theta} \circ \delta((1, \ldots, 1, \Sigma q, 1, \ldots, 1) \sigma(1, \ldots, 1, \Sigma p, 1, \ldots, 1)) \\
& =d_{\theta}(-(1, \ldots, 1, \Sigma \delta(q), 1, \ldots, 1) \sigma(1, \ldots, 1, \Sigma p, 1, \ldots, 1) \\
& \left.+(-1)^{|q|}(1, \ldots, 1, \Sigma q, 1, \ldots, 1) \sigma(1, \ldots, 1, \Sigma \delta(p), 1, \ldots, 1)\right) \\
& =(-1)^{|q|} \Sigma \mu_{(1,1)}((1, \ldots, 1, \delta(q), 1, \ldots, 1) \sigma(1, \ldots, 1, p, 1, \ldots, 1)) \\
& +\Sigma \mu_{(1,1)}((1, \ldots, 1, q, 1, \ldots, 1) \sigma(1, \ldots, 1, \delta(p), 1, \ldots, 1)) .
\end{aligned}
$$

Therefore, $\delta d_{\theta}+d_{\theta} \delta=0$ implies that

$$
\begin{aligned}
& \delta\left(\mu_{(1,1)}((1, \ldots, 1, q, 1, \ldots, 1) \sigma(1, \ldots, 1, p, 1, \ldots, 1))\right) \\
& =\mu_{(1,1)}((1, \ldots, 1, \delta(q), 1, \ldots, 1) \sigma(1, \ldots, 1, p, 1, \ldots, 1)) \\
& +(-1)^{|q|} \mu_{(1,1)}((1, \ldots, 1, q, 1, \ldots, 1) \sigma(1, \ldots, 1, \delta(p), 1, \ldots, 1)),
\end{aligned}
$$

which means that the partial composition product $\mu_{(1,1)}$ is a morphism of a dg-S-bimodule. In the other way, applying $\delta d_{\theta}+d_{\theta} \delta$ corresponds to do a sum of expressions of the previous type.

(2) To show that $d_{\theta}^{2}=0$, we have to consider the pairs of couples of vertices of a graph $g$. There are two possible cases : the pairs of couples of vertices such that the four vertices are different (a), the pairs of couples of vertices with one common vertex (b). 
(a) To an element of the form

$$
X \otimes \Sigma q_{1} \otimes \Sigma p_{1} \otimes Y \otimes \Sigma q_{2} \otimes \Sigma p_{2} \otimes Z,
$$

we apply $\theta$ twice beginning with one different couple each time. It gives

$\left((-1)^{|X|+\left|q_{1}\right|}(-1)^{|X|+\left|q_{1}\right|+\left|p_{1}\right|+1+|Y|+\left|q_{2}\right|}+(-1)^{|X|+\left|q_{1}\right|+\left|p_{1}\right|+|Y|+\left|q_{2}\right|}(-1)^{|X|+\left|q_{1}\right|}\right)$ $\times X \otimes \Sigma \mu_{(1,1)}\left(q_{1} \otimes p_{1}\right) \otimes Y \otimes \Sigma \mu_{(1,1)}\left(q_{2} \otimes p_{2}\right) \otimes Z=0$.

(b) In this case, there are two possible configurations:

- The common vertex is between the two other vertices (following the flow of the graph) ( $c f$. Figure 4).

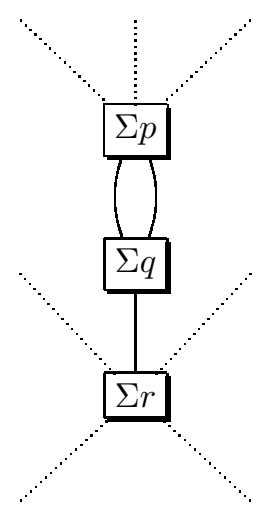

FigURE 4.

Therefore, to an element of the form

$$
X \otimes \Sigma r \otimes \Sigma q \otimes \Sigma p \otimes Y,
$$

if we apply $\theta$ twice beginning by a different couple each time, we have

$$
\begin{aligned}
& (-1)^{|X|+|r|}(-1)^{|X|+|r|+|q|} X \otimes \Sigma \mu_{(1,1)}\left(\mu_{(1,1)}(r \otimes q) \otimes p\right) \otimes Y \\
& +(-1)^{|X|+|r|+1+|q|}(-1)^{|X|+|r|} X \otimes \Sigma \mu_{(1,1)}\left(r \otimes \mu_{(1,1)}(q \otimes p)\right) \otimes Y=0,
\end{aligned}
$$

by associativity of the partial product $\mu_{(1,1)}$ (which comes from the associativity of $\mu$ ).

- Otherwise, the common vertex is above (cf. Figure 5) or under the two others.

The same kind of calculus this time gives

$$
\begin{aligned}
& (-1)^{|X|+|r|+1+|q|}(-1)^{|X|+|r|} X \otimes \Sigma \mu_{(1,1)}\left(r \otimes \mu_{(1,1)}(q \otimes p)\right) \otimes Y \\
& +(-1)^{(|r|)+1(|q|+1)+|X|+|q|+1+|r|}(-1)^{|X|+|q|} X \otimes \Sigma \mu_{(1,1)}\left(q \otimes \mu_{(1,1)}(r \otimes p)\right) \otimes Y=0 .
\end{aligned}
$$

Also, the associativity of $\mu_{(1,1)}$ gives

$$
\mu_{(1,1)}\left(q \otimes \mu_{(1,1)}(r \otimes p)\right)=(-1)^{|r||q|} \mu_{(1,1)}\left(r \otimes \mu_{(1,1)}(q \otimes p)\right) .
$$

We conclude by remarking that the image under the morphism $d_{\theta}^{2}$ is a sum of expressions of such type. 


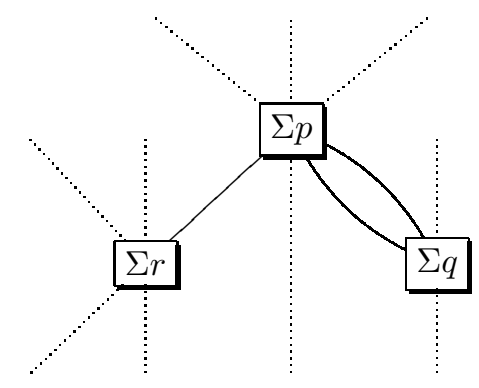

FiguRE 5 .

Remark. This proposition lies on the natural Koszul-Quillen sign rules. In the article of [GK], these signs appear via the operad Det.

Definition (Pair of adjacent vertices). Any pair of vertices of a graph linked by at least on edge and without a vertex between is called a pair of adjacent vertices. It corresponds to sub-graphs of the form $\mathcal{F}_{(2)}(V)=\mathcal{F}_{(2)}^{c}(V)$ and are the composable pairs under the coderivation $d_{\theta}$.

Remark. The image under the coderivation $d_{\theta}$ is given by the composition of all pairs of adjacent vertices. In order to define the homology of graphs, M. Kontsevich has introduced in $[\mathrm{Ko}$ the notion of an edge contraction. This notion corresponds to the composition of a pair of vertices linked by only one edge. In the case of operads (trees), $\frac{1}{2}$-PROPs and dioperads (graphs of genus 0 ), adjacent vertices are linked by only one edge. Therefore, this notion applies there. But in the framework of properads, this notion is too restrictive and its natural generalization is given by the coderivation $d_{\theta}$.

4.1.3. Partial coproduct. Here we dualize the results of the previous section.

Definition (Partial coproduct). Let $(\mathcal{C}, \Delta, \varepsilon, \eta)$ be a coaugmented coproperad. The following composition is called a partial coproduct:

$$
\overline{\mathcal{C}} \stackrel{\Delta}{\rightarrow} \mathcal{C} \otimes_{c} \mathcal{C} \rightarrow(I \oplus \underbrace{\overline{\mathcal{C}}}_{1}) \otimes_{c}(I \oplus \underbrace{\overline{\mathcal{C}}}_{1}) .
$$

It corresponds to the part of the image of $\Delta$ represented by graphs with two vertices. We denote it as $\Delta_{(1,1)}$.

Lemma 4.3. Let $(\mathcal{C}, \Delta, \varepsilon, \eta)$ be a differential coaugmented coproperad. The partial coproduct induces an homogenous morphism of degree -1

$$
\theta^{\prime}: \Sigma^{-1} \overline{\mathcal{C}} \rightarrow \mathcal{F}_{(2)}\left(\Sigma^{-1} \overline{\mathcal{C}}\right)=(I \oplus \underbrace{\overline{\mathcal{C}}}_{1}) \otimes_{c}(I \oplus \underbrace{\overline{\mathcal{C}}}_{1})
$$

Proof. Let $c$ be an element of $\overline{\mathcal{C}}$. Using the same kind of notations as Sweedler for Hopf algebras, we have

$$
\Delta_{(1,1)}(c)=\sum_{\left(c^{\prime}, c^{\prime \prime}\right)}\left(1, \ldots, 1, c^{\prime}, 1, \ldots, 1\right) \sigma\left(1, \ldots, 1, c^{\prime \prime}, 1, \ldots, 1\right) .
$$


Hence, we define $\theta^{\prime}\left(\Sigma^{-1} c\right)$ by the formula $\theta^{\prime}\left(\Sigma^{-1} c\right):=-\sum_{\left(c^{\prime}, c^{\prime \prime}\right)}(-1)^{\left|c^{\prime}\right|}\left(1, \ldots, 1, \Sigma^{-1} c^{\prime}, 1, \ldots, 1\right) \sigma\left(1, \ldots, 1, \Sigma^{-1} c^{\prime \prime}, 1, \ldots, 1\right)$.

Remark. The sign - appearing in the definition of $\theta^{\prime}$ is not essential here. Its role will be obvious in the proof of the bar-cobar resolution ( $c f$. Theorem 5.8).

With Proposition 3.14, we can associate a derivation $d_{\theta^{\prime}}: \mathcal{F}\left(\Sigma^{-1} \overline{\mathcal{C}}\right) \rightarrow \mathcal{F}\left(\Sigma^{-1} \overline{\mathcal{C}}\right)$ of degree -1 to the homogenous morphism $\theta^{\prime}$ which verifies the following properties.

\section{Proposition 4.4.}

(1) The equation $\delta d_{\theta^{\prime}}+d_{\theta^{\prime}} \delta=0$ is true if and only if the partial coproduct $\Delta_{(1,1)}$ is a morphism of $d g-\mathbb{S}$-bimodules.

(2) We have $d_{\theta^{\prime}}{ }^{2}=0$.

Proof. The proof of Proposition 3.14 shows that the image of an element of $\mathcal{F}\left(\Sigma^{-1} \overline{\mathcal{C}}\right)$ represented by a graph $g$ under the derivation $d_{\theta^{\prime}}$ is a sum where the application $\theta^{\prime}$ is applied on each vertex of $g$. Therefore, the arguments to show this proposition are the same as for the previous proposition. For instance, the second point comes from the coassociativity of $\Delta_{(1,1)}$, which is induced by the one of $\Delta$, and from the rules of signs.

Remark. The derivation $d_{\theta^{\prime}}$ corresponds to taking the partial coproduct of every element indexing a vertex of a graph. Therefore, this derivation is the natural generalization of the notion of vertex expansion introduced by M. Kontsevich in [Ko in the framework of graph cohomology. In the case of operads, the derivation $d_{\theta^{\prime}}$ is the same morphism as the one defined by the "vertex expansion" of trees.

4.2. Definitions of the bar and cobar constructions. With the propositions given above, we can now define the bar and cobar constructions for properads.

\subsubsection{Reduced bar and cobar constructions.}

Definition (Reduced bar construction). To any differential augmented properad $(\mathcal{P}, \mu, \eta, \varepsilon)$, we can associate the quasi-cofree coproperad $\overline{\mathcal{B}}(\mathcal{P}):=\mathcal{F}^{c}(\Sigma \overline{\mathcal{P}})$ with the differential defined by $\delta_{\theta}=\delta+d_{\theta}$, where $\theta$ is the morphism induced by the partial product of $\mathcal{P}$ ( $c f$. Lemma 4.1). This quasi-cofree coproperad is called the reduced bar construction of $\mathcal{P}$.

We denote $\overline{\mathcal{B}}_{(s)}(\mathcal{P}):=\mathcal{F}_{(s)}^{c}(\Sigma \overline{\mathcal{P}})$. Therefore, $d_{\theta}$ defines the following chain complex:

$$
\cdots \stackrel{d_{\theta}}{\longrightarrow} \overline{\mathcal{B}}_{(s)}(\mathcal{P}) \stackrel{d_{\theta}}{\longrightarrow} \overline{\mathcal{B}}_{(s-1)}(\mathcal{P}) \stackrel{d_{\theta}}{\longrightarrow} \cdots \stackrel{d_{\theta}}{\longrightarrow} \overline{\mathcal{B}}_{(1)}(\mathcal{P}) \stackrel{d_{\theta}}{\longrightarrow} \overline{\mathcal{B}}_{(0)}(\mathcal{P}) .
$$

Proposition 4.2 gives that $\delta d_{\theta}+d_{\theta} \delta=0$ and that $d_{\theta}{ }^{2}=0$. Hence, we have $\delta_{\theta}{ }^{2}=0$ and we see that the reduced bar construction is the total complex of a bicomplex.

Dually, we have the following definition:

Definition (Reduced cobar construction). To any differential coaugmented coproperad $(\mathcal{C}, \Delta, \varepsilon, \eta)$ we can associate the quasi-free properad $\overline{\mathcal{B}}^{c}(\mathcal{C}):=\mathcal{F}\left(\Sigma^{-1} \overline{\mathcal{C}}\right)$ with the differential defined by $\delta_{\theta^{\prime}}=\delta+d_{\theta^{\prime}}$, where $\theta^{\prime}$ is the morphism induced by 
the partial coproduct of $\mathcal{C}(c f$. Lemma 4.3). This quasi-free properad is called the reduced cobar construction of $\mathcal{C}$.

Once again, the derivation $d_{\theta^{\prime}}$ defines a cochain complex

$$
\overline{\mathcal{B}}_{(0)}^{c}(\mathcal{C}) \stackrel{d_{\theta^{\prime}}}{\longrightarrow} \overline{\mathcal{B}}_{(1)}^{c}(\mathcal{C}) \stackrel{d_{\theta^{\prime}}}{\longrightarrow} \cdots \stackrel{d_{\theta^{\prime}}}{\longrightarrow} \overline{\mathcal{B}}_{(s)}^{c}(\mathcal{C}) \stackrel{d_{\theta^{\prime}}}{\longrightarrow} \overline{\mathcal{B}}_{(s+1)}^{c}(\mathcal{C}) \stackrel{d_{\theta^{\prime}}}{\longrightarrow} \cdots .
$$

The reduced cobar construction is the total complex of a bicomplex.

Remark. When $\mathcal{P}$ (or $\mathcal{C}$ ) is an algebra or an operad (a coalgebra or a cooperad), we find the classical definitions of bar and cobar construction ( $c f$. Séminaire H. Cartan [C] and [GK]).

4.2.2. Bar construction with coefficients. Let $(\mathcal{P}, \mu, \eta, \varepsilon)$ be an augmented differential properad. On $\overline{\mathcal{B}}(\mathcal{P})$ we can define two homogenous morphisms $\theta_{r}$ : $\overline{\mathcal{B}}(\mathcal{P}) \rightarrow \overline{\mathcal{B}}(\mathcal{P}) \otimes_{c} \mathcal{P}$ and $\theta_{l}: \overline{\mathcal{B}}(\mathcal{P}) \rightarrow \mathcal{P} \otimes_{c} \overline{\mathcal{B}}(\mathcal{P})$ of degree -1 by

$$
\theta_{r}: \quad \overline{\mathcal{B}}(\mathcal{P})=\mathcal{F}^{c}(\Sigma \overline{\mathcal{P}}) \stackrel{\Delta}{\longrightarrow} \mathcal{F}^{c}(\Sigma \overline{\mathcal{P}}) \otimes_{c} \mathcal{F}^{c}(\Sigma \overline{\mathcal{P}}) \rightarrow \mathcal{F}^{c}(\Sigma \overline{\mathcal{P}}) \otimes_{c}(I \oplus \underbrace{\overline{\mathcal{P}}}_{1}),
$$

and by

$$
\theta_{l}: \overline{\mathcal{B}}(\mathcal{P})=\mathcal{F}^{c}(\Sigma \overline{\mathcal{P}}) \stackrel{\Delta}{\longrightarrow} \mathcal{F}^{c}(\Sigma \overline{\mathcal{P}}) \bigotimes_{c} \mathcal{F}^{c}(\Sigma \overline{\mathcal{P}}) \rightarrow(I \oplus \underbrace{\overline{\mathcal{P}}}_{1}) \bigotimes_{c} \mathcal{F}^{c}(\Sigma \overline{\mathcal{P}}) .
$$

Recall that these two morphisms correspond to extract one non-trivial operation from the top or the bottom of graphs that represent elements of $\mathcal{F}^{c}(\Sigma \overline{\mathcal{P}})$.

Lemma 4.5. The morphism $\theta_{r}$ induces a homogenous morphism $d_{\theta_{r}}$ of degree -1 :

$$
d_{\theta_{r}}: \overline{\mathcal{B}}(\mathcal{P}) \otimes_{c} \mathcal{P} \rightarrow \overline{\mathcal{B}}(\mathcal{P}) \otimes_{c} \mathcal{P} .
$$

Also, the dg-S-bimodule $\overline{\mathcal{B}}(\mathcal{P}) \bigotimes_{c} \mathcal{P}$ endowed with the differential defined by the sum of the canonical differential $\delta$ with the coderivation $d_{\theta}$ of $\overline{\mathcal{B}}(\mathcal{P})$ and the morphism $d_{\theta_{r}}$ is an analytic quasi-free $\mathcal{P}$-module (on the right).

Proof. Since $\left(\delta+d_{\theta}\right)^{2}=0$, the equation $\left(\delta+d_{\theta}+d_{\theta_{r}}\right)^{2}=0$ is equivalent to

$$
\left\{\begin{array}{l}
\left(\delta+d_{\theta}\right) d_{\theta_{r}}+d_{\theta_{r}}\left(\delta+d_{\theta}\right)=0 \text { and } \\
d_{\theta_{r}}{ }^{2}=0 .
\end{array}\right.
$$

These two equations are proved in the same arguments as in the proof of Proposition 4.2. The functor $\overline{\mathcal{B}}(\mathcal{P})$ is analytic in $\overline{\mathcal{P}}$, therefore the quasi-free $\mathcal{P}$-module $\overline{\mathcal{B}}(\mathcal{P}) \otimes_{c} \mathcal{P}$ is analytic.

The morphism $d_{\theta_{r}}$ corresponds to taking one by one operations indexing top vertices of the graph of an element of $\overline{\mathcal{B}}(\mathcal{P})$ and composing them with operations of $\mathcal{P}$ that are on the first level of $\overline{\mathcal{B}}(\mathcal{P}) \otimes_{c} \mathcal{P}$.

Definition (Augmented bar construction). The analytic quasi-free $\mathcal{P}$-module $\overline{\mathcal{B}}(\mathcal{P})$ $\otimes_{c} \mathcal{P}$ is called the augmented bar construction (on the right).

One has the same lemma with $\theta_{l}$ and $d_{\theta_{l}}$ and defines the augmented bar construction on the left $\mathcal{P} \otimes_{c} \overline{\mathcal{B}}(\mathcal{P})$.

In the case of algebras and operads, the augmented bar construction is acyclic. In the following part of the text, we generalize this result to the augmented bar construction of properads. 
Denote $\mathcal{B}(\mathcal{P}, \mathcal{P}, \mathcal{P}):=\mathcal{P} \otimes_{c} \overline{\mathcal{B}}(\mathcal{P}) \otimes_{c} \mathcal{P}$. The morphisms $\theta_{r}$ and $\theta_{l}$ induce two homogenous morphisms

$$
d_{\theta_{r}}, d_{\theta_{l}}: B(\mathcal{P}, \mathcal{P}, \mathcal{P}) \rightarrow \mathcal{B}(\mathcal{P}, \mathcal{P}, \mathcal{P}),
$$

of degree -1 . We define the differential $d$ on $\mathcal{B}(\mathcal{P}, \mathcal{P}, \mathcal{P})$ by the sum of the following morphisms :

- the canonical differential $\delta$ induced by the one of $\mathcal{P}$,

- the coderivation $d_{\theta}$ defined on $\overline{\mathcal{B}}(\mathcal{P})$,

- the homogenous morphism $d_{\theta_{r}}$ of degree -1 ,

- the homogenous morphism $d_{\theta_{r}}$ of degree -1 .

Lemma 4.6. The morphism $d$ verifies the equation $d^{2}=0$.

Proof. Once again, the proof lies on rules of sign.

Definition (Bar construction with coefficients). Let $(\mathcal{P}, \mu, \eta, \varepsilon)$ be an augmented dg-properad, $L$ be a differential right $\mathcal{P}$-module and $R$ be a differential left $\mathcal{P}$ module. The bar construction of $\mathcal{P}$ with coefficients in $L$ and $R$ is the dg- $\mathbb{S}$-bimodule $\mathcal{B}(L, \mathcal{P}, R):=L \bigotimes_{\mathcal{C}} \mathcal{P}(\mathcal{P}, \mathcal{P}, \mathcal{P}) \bigotimes_{c \mathcal{P}} R$, where the differential $d$ is induced by the one of $\mathcal{B}(\mathcal{P}, \mathcal{P}, \mathcal{P})$, defined previously, and by the ones of $L$ and $R$ denoted as $\delta_{R}$ and $\delta_{L}$.

Proposition 4.7. The bar construction $\mathcal{B}(L, \mathcal{P}, R)$ with coefficients in $L$ and $R$ is isomorphic, as a $d g-\mathbb{S}$-bimodule, to $L \bigotimes_{c} \overline{\mathcal{B}}(\mathcal{P}) \bigotimes_{c} R$, where the differential $d$ is defined by the sum of the following morphisms:

- the canonical differential $\delta$ induced by the one of $\mathcal{P}$ on $\overline{\mathcal{B}}(\mathcal{P})$,

- the canonical differential $\delta_{L}$ induced by the one of $L$,

- the canonical differential $\delta_{R}$ induced by the one of $R$,

- the coderivation $d_{\theta}$ defined on $\overline{\mathcal{B}}(\mathcal{P})$,

- $\quad$ a homogenous morphism $d_{\theta_{R}}$ of degree -1 ,

- $\quad$ a homogenous morphism $d_{\theta_{L}}$ of degree -1 .

Proof. One has to understand the effect of the morphism $d_{\theta_{r}}$ on the relative composition product. The morphism $d_{\theta_{r}}$ corresponds to the morphism $d_{\theta_{R}}$ defined by the following composition:

$$
L \bigotimes_{c} \overline{\mathcal{B}}(\mathcal{P}) \bigotimes_{c} R \stackrel{\widetilde{\theta_{r}}}{\longrightarrow} L \bigotimes_{c} \overline{\mathcal{B}}(\mathcal{P}) \bigotimes_{c} \mathcal{P} \bigotimes_{c} R \stackrel{L \bigotimes_{c} \overline{\mathcal{B}}(\mathcal{P}) \bigotimes_{c} r}{\longrightarrow} L \bigotimes_{c} \overline{\mathcal{B}}(\mathcal{P}) \bigotimes_{c} R,
$$

where the morphism $\tilde{\theta}_{r}$ is induced by $\theta_{r}$.

From this description of the bar construction with coefficients, we immediately have the following corollary.

\section{Corollary 4.8 .}

- The reduced bar construction is isomorphic to the bar construction with scalar coefficients $L=R=I$. We have $\overline{\mathcal{B}}(\mathcal{P})=\mathcal{B}(I, \mathcal{P}, I)$.

- The chain complex $\mathcal{B}(L, \mathcal{P}, \mathcal{P})=L \otimes_{c} \overline{\mathcal{B}}(\mathcal{P}) \otimes_{c} \mathcal{P}$ (resp. $\mathcal{B}(\mathcal{P}, \mathcal{P}, R)=$ $\mathcal{P} \otimes_{c} \overline{\mathcal{B}}(\mathcal{P}) \bigotimes_{c} R$ ) is an analytic quasi-free $\mathcal{P}$-module on the right (resp. on the left). 
In the same way as Proposition 3.13 and Theorem 5.9, we have that the bar construction is an exact functor.

Proposition 4.9. The bar construction $\mathcal{B}(L, \mathcal{P}, R)$ preserves quasi-isomorphisms.

Proof. Let $\beta: \mathcal{P} \rightarrow \mathcal{P}^{\prime}$ be a quasi-isomorphism of augmented dg-properads. Let $\alpha: L \rightarrow L^{\prime}$ be a quasi-isomorphism of right $\operatorname{dg}-\mathcal{P}, \mathcal{P}^{\prime}$-modules and let $\gamma: R \rightarrow$ $R^{\prime}$ be a quasi-isomorphism of left dg- $\mathcal{P}, \mathcal{P}^{\prime}$-modules. Denote by $\Phi$ the induced morphism $\mathcal{B}(L, \mathcal{P}, R) \rightarrow \mathcal{B}\left(L^{\prime}, \mathcal{P}^{\prime}, R^{\prime}\right)$. To show that $\Phi$ is a quasi-isomorphism, we introduce the following filtration $F_{r}:=\bigoplus_{s \leq r} \mathcal{B}_{(s)}(L, \mathcal{P}, R)$, where $s$ is the number of vertices of the underlying graph indexed by elements of $\Sigma \overline{\mathcal{P}}$. One can see that this filtration is stable under the differential $d$ of the bar construction. The canonical differentials $\delta, \delta_{L}$ and $\delta_{R}$ go from $\mathcal{B}_{(s)}(L, \mathcal{P}, R)$ to $\mathcal{B}_{(s)}(L, \mathcal{P}, R)$ and the maps $d_{\theta}, d_{\theta_{R}}$ and $d_{\theta_{R}}$ go from $\mathcal{B}_{(s)}(L, \mathcal{P}, R)$ to $\mathcal{B}_{(s-1)}(L, \mathcal{P}, R)$. Therefore, this filtration induces a spectral sequence $E_{s, t}^{*}$ such that $E_{s, t}^{0}=\mathcal{B}_{(s)}(L, \mathcal{P}, R)_{s+t}$ and such that $d^{0}=\delta+\delta_{L}+\delta_{R}$. By the same ideas as in the proof of Proposition 3.13. we have that $E_{s, t}^{0}(\Phi)$ is a quasi-isomorphism. Since the filtration $F_{r}$ is bounded below and exhaustive, the result is given by the classical convergence theorem of spectral sequences ( $c f$. J.A. Weibel W] 5.5.1).

4.2.3. Cobar construction with coefficients. We dualize the previous arguments to define the cobar construction with coefficients.

Let $(\mathcal{C}, \Delta, \varepsilon, \eta)$ be a differential coaugmented coproperad and let $(L, l)$ and $(R, r)$ be two differential $\mathcal{C}$-comodules on the right and on the left.

The two $\mathcal{C}$-comodules induce the following morphisms:

$$
\begin{aligned}
\theta_{R}^{\prime} & : R \stackrel{r}{\rightarrow} \mathcal{C} \bigotimes_{c} R \rightarrow(I \oplus \underbrace{\overline{\mathcal{C}}}_{1}) \bigotimes_{c} R, \\
\theta_{L}^{\prime} & : L \stackrel{r}{\rightarrow} L \bigotimes_{c} \mathcal{C} \rightarrow L \bigotimes_{c}(I \oplus \underbrace{\overline{\mathcal{C}}}_{1}) .
\end{aligned}
$$

Lemma 4.10. The applications $\theta_{R}^{\prime}$ and $\theta_{L}^{\prime}$ both induce a homogenous morphism of degree -1 on $L \otimes_{c} \overline{\mathcal{B}}^{c}(\mathcal{C}) \bigotimes_{c} R$ of the form

$$
\begin{aligned}
& d_{\theta_{R}^{\prime}} \quad: \quad L \bigotimes_{c} \overline{\mathcal{B}}^{c}(\mathcal{C}) \bigotimes_{c} R \stackrel{\widetilde{\theta_{R}^{\prime}}}{\longrightarrow} \quad L \bigotimes_{c} \overline{\mathcal{B}}^{c}(\mathcal{C}) \bigotimes_{c}(I \oplus \underbrace{\overline{\mathcal{C}}}_{1}) \bigotimes_{c} R \\
& \longrightarrow L \bigotimes_{c} \overline{\mathcal{B}}^{c}(\mathcal{C}) \bigotimes_{c}(I \oplus \underbrace{\Sigma^{-1} \overline{\mathcal{C}}}_{1}) \bigotimes_{c} R \stackrel{L \bigotimes_{c} \mu_{\overline{\mathcal{B}}^{c}(\mathcal{C})} \bigotimes_{c} R}{\longrightarrow} L \bigotimes_{c} \overline{\mathcal{B}}^{c}(\mathcal{C}) \bigotimes_{c} R, \\
& \theta_{L}^{\prime} \quad: \quad d_{\theta_{L}^{\prime}}: L \bigotimes_{c} \overline{\mathcal{B}}^{c}(\mathcal{C}) \bigotimes_{c} R \stackrel{\widetilde{\theta_{L}^{\prime}}}{\longrightarrow} L \bigotimes_{c}(I \oplus \underbrace{\overline{\mathcal{C}}}_{1}) \bigotimes_{c} \overline{\mathcal{B}}^{c}(\mathcal{C}) \bigotimes_{c} R \\
& \longrightarrow L \bigotimes_{c}(I \oplus \underbrace{\Sigma^{-1} \overline{\mathcal{C}}}_{1}) \bigotimes_{c} \overline{\mathcal{B}}^{c}(\mathcal{C}) \bigotimes_{c} R \stackrel{L \bigotimes_{c} \mu_{\overline{\mathcal{B}}^{c}(\mathcal{C})} \bigotimes_{c} R}{\longrightarrow} L \bigotimes_{c} \overline{\mathcal{B}}^{c}(\mathcal{C}) \bigotimes_{c} R
\end{aligned}
$$


Proposition 4.11. The differential $d$ on the $\mathbb{S}$-bimodule $L \nabla_{c} \overline{\mathcal{B}}^{c}(\mathcal{C}) \bigotimes_{c} R$ is the sum of the following morphisms :

- $\quad$ the canonical differential $\delta$ induced by the one of $\mathcal{C}$ on $\overline{\mathcal{B}}^{c}(\mathcal{C})$,

- the canonical differential $\delta_{L}$ induced by the one of $L$,

- the canonical differential $\delta_{R}$ induced by the one of $R$,

- the derivation $d_{\theta}$ defined on $\overline{\mathcal{B}}^{c}(\mathcal{C})$,

- the homogenous morphism $d_{\theta_{R}^{\prime}}$ of degree -1 ,

- the homogenous morphism $d_{\theta_{L}^{\prime}}$ of degree -1 .

Definition (Cobar construction with coefficients). The dg-SS-bimodule $L \bigotimes_{c} \overline{\mathcal{B}}^{c}(\mathcal{C}) \bigotimes_{c}$ $R$ endowed with the differential $d$ is called the cobar construction with coefficients in $L$ and $R$ and is denoted $\mathcal{B}^{c}(L, \mathcal{C}, R)$.

\section{Corollary 4.12 .}

- The reduced cobar construction $\overline{\mathcal{B}}^{c}(\mathcal{C})$ is isomorphic to the cobar construction with coefficients in the scalar $\mathcal{C}$-comodule $I$.

- The chain complex $\mathcal{B}^{c}(L, \mathcal{C}, \mathcal{C})=L \bigotimes_{c} \overline{\mathcal{B}}^{c}(\mathcal{C}) \bigotimes_{c} \mathcal{C}$ (resp. $\mathcal{B}(\mathcal{C}, \mathcal{C}, R)=$ $\mathcal{C} \otimes_{c} \overline{\mathcal{B}}^{c}(\mathcal{C}) \bigotimes_{c} R$ ) is an analytic quasi-cofree $\mathcal{C}$-comodule on the right (resp. on the left)

4.3. Acyclicity of the augmented bar and cobar constructions. When $\mathcal{P}$ is an algebra $A$, that's to say when the dg- $\mathbb{S}$-bimodule $\mathcal{P}$ is concentrated in $\mathcal{P}(1,1)=$ $A$, one has that the augmented bar construction is acyclic. To prove it, one directly introduces a contracting homotopy ( $c f$. Séminaire H. Cartan $[\mathrm{C}$ ). In the case of operads, B. Fresse has proved that the augmented bar construction on the left $\mathcal{P} \circ \overline{\mathcal{B}}(\mathcal{P})$ is acyclic using a contracting homotopy. This homotopy lies on the fact the monoidal product $\circ$ is linear on the left. The acyclicity of the augmented bar construction on the right comes from this result and the use of comparison lemmas for quasi-free modules ( $c f$. $[\mathrm{Fr}$ ] section 4.6).

In the framework of properads, since the composition product $\otimes_{c}$ is not linear on the left or on the right, we cannot use the same method. We begin by defining a filtration on the chain complex $\left(\mathcal{P} \otimes_{c} \overline{\mathcal{B}}(\mathcal{P}), d\right)$. This filtration induces a convergent spectral sequence. Then, we introduce a contracting homotopy on the chain complexes $\left(E_{p, *}^{0}, d^{0}\right)$, for $p>0$, to show that they are acyclic.

4.3.1. Acyclicity of the augmented bar construction. Let $(\mathcal{P}, \mu, \eta, \varepsilon)$ be a augmented dg-properad. The dg-S-bimodule $\mathcal{P} \otimes_{c} \overline{\mathcal{B}}(\mathcal{P})$ is the image of the functor

$$
\overline{\mathcal{P}} \mapsto(I \oplus \overline{\mathcal{P}}) \bigotimes_{c} \mathcal{F}^{c}(\Sigma \overline{\mathcal{P}}) .
$$

Since this functor is a sum of polynomial functors, it is a split analytic functor (cf. 2.7). For an element of $(\mathcal{P}, \mu, \eta, \varepsilon)$, the graduation is given by the number of vertices indexed by elements of $\overline{\mathcal{P}}$. Once again, we denote this (total) graduation by

$$
\mathcal{P} \otimes_{c} \overline{\mathcal{B}}(\mathcal{P})=\bigoplus_{s \in \mathbb{N}}\left(\mathcal{P} \bigotimes_{c} \overline{\mathcal{B}}(\mathcal{P})\right)_{(s)} .
$$

We consider the filtration defined by

$$
F_{i}=F_{i}(\mathcal{P} \otimes \overline{\mathcal{B}}(\mathcal{P})):=\bigoplus_{s \leq i}\left(\mathcal{P} \otimes_{c} \overline{\mathcal{B}}(\mathcal{P})\right)_{(s)} .
$$


Therefore, $F_{i}$ is composed by elements $\mathcal{P} \otimes \overline{\mathcal{B}}(\mathcal{P})$ represented by graphs indexed at most $i$ elements of $\overline{\mathcal{P}}$.

Lemma 4.13. The filtration $F_{i}$ of the $d g$-S-bimodule $\mathcal{P} \otimes_{c} \overline{\mathcal{B}}(\mathcal{P})$ is stable under the differential d of the augmented bar construction.

Proof. Lemma 4.5 gives the form of the differential $d$ of the augmented bar construction. It is the sum of the three following terms :

(1) The differential $\delta$ coming from the one of $\mathcal{P}$. This one does not change the number of elements of $\overline{\mathcal{P}}$. Hence, one has $\delta\left(F_{i}\right) \subset F_{i}$.

(2) The coderivation $d_{\theta}$ of the reduced bar construction $\overline{\mathcal{B}}(\mathcal{P})$. This morphism corresponds to the composition of pairs of adjacent vertices. Therefore, it verifies $d_{\theta}\left(F_{i}\right) \subset F_{i-1}$.

(3) The morphism $d_{\theta_{l}}$. Applying this morphism corresponds to taking an operation of $\Sigma \overline{\mathcal{P}}$ of $\overline{\mathcal{B}}(\mathcal{P})$ from the bottom and composing it with elements of $\mathcal{P}$ on the last level of the graph,

$$
\mathcal{P} \otimes_{c} \overline{\mathcal{B}}(\mathcal{P}) \rightarrow \mathcal{P} \otimes_{c}(I \oplus \overline{\mathcal{P}}) \bigotimes_{c} \overline{\mathcal{B}}(\mathcal{P}) \rightarrow \mathcal{P} \otimes_{c} \overline{\mathcal{B}}(\mathcal{P}) .
$$

The total number of elements of $\overline{\mathcal{P}}$ is decreasing, and one has $d_{\theta_{l}}\left(F_{i}\right) \subset F_{i}$.

This lemma shows that the filtration $F_{i}$ induces a spectral sequence, denoted as $E_{p, q}^{*}$. The first term is equal to

$$
E_{p, q}^{0}=F_{p}\left(\left(\mathcal{P} \otimes_{c} \overline{\mathcal{B}}(\mathcal{P})\right)_{p+q}\right) / F_{p-1}\left(\left(\mathcal{P} \otimes_{c} \overline{\mathcal{B}}(\mathcal{P})\right)_{p+q}\right),
$$

where $p+q$ is the homological degree. Hence, the module $E_{p, q}^{0}$ is given by the graphs with exactly $p$ vertices indexed by elements of $\overline{\mathcal{P}}$. We have

$$
E_{p, q}^{0}=\left(\left(\mathcal{P} \otimes_{c} \overline{\mathcal{B}}(\mathcal{P})\right)_{(p)}\right)_{p+q}
$$

and the differential $d^{0}$ is a sum of the morphisms $d^{0}=\delta+d_{\theta_{l}}^{\prime}$. The morphism $\delta$ is induced by the one of $\mathcal{P}$, and the morphism $d_{\theta_{l}}^{\prime}$ is equal to the morphism $d_{\theta_{l}}$ when it does not change the total number of vertices indexed by $\overline{\mathcal{P}}$. Therefore, the morphism $d_{\theta_{l}}^{\prime}$ takes one operation of $\Sigma \overline{\mathcal{P}}$ in $\overline{\mathcal{B}}(\mathcal{P})$ from the bottom and inserts it on the line of elements of $\mathcal{P}$ (in the last level) without composing it with operations of $\overline{\mathcal{P}}$. Otherwise, the morphism $d_{\theta_{l}}$ implies a non-trivial composition with elements of $\mathcal{P}$, in this case $d_{\theta_{l}}^{\prime}$ is null ( $c f$. figure 6 ).

The spectral sequence $E_{p, q}^{*}$ is concentrated in the half-plane $p \geq 0$. We compute the homology of the chain complexes $\left(E_{p, *}^{0}, d^{0}\right)$ to show that the spectral sequence collapses at rank $E_{p, q}^{1}$.

Lemma 4.14. At rank $E_{p, q}^{1}$, we have

$$
E_{p, q}^{1}= \begin{cases}I & \text { if } p=q=0 \\ 0 & \text { otherwise }\end{cases}
$$

Proof. When $p=0$, we have

$$
E_{0, q}^{0}= \begin{cases}I & \text { if } q=0 \\ 0 & \text { otherwise }\end{cases}
$$




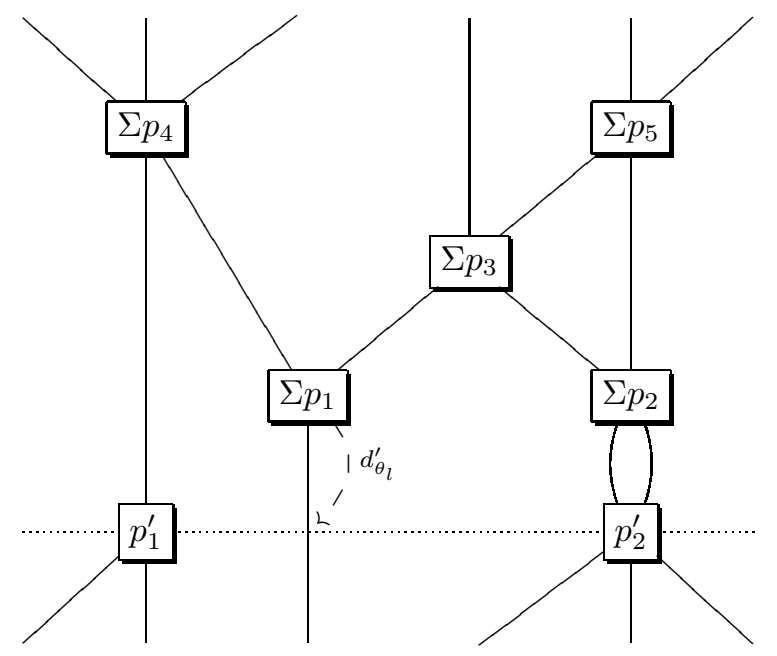

FIGURE 6. Form of the morphism $d_{\theta_{l}}^{\prime}$

and the differential $d^{0}$ is null on the modules $E_{0, q}^{0}$. Hence, the homology of these modules is equal to

$$
E_{0, q}^{1}= \begin{cases}I & \text { if } q=0 \\ 0 & \text { otherwise. }\end{cases}
$$

When $p>0$, we introduce a contracting homotopy $h$ for each chain of complexes $\left(E_{p, *}^{0}, d^{0}\right)$.

Thanks to Proposition 3.2, we can represent an element of $\mathcal{P} \otimes \overline{\mathcal{B}}(\mathcal{P})$ by $\left(p_{1}, \ldots\right.$, $\left.p_{r}\right) \sigma\left(b_{1}, \ldots, b_{s}\right)$. When $p_{1} \in I$, we define

$$
h\left(\left(p_{1}, \ldots, p_{r}\right) \sigma\left(b_{1}, \ldots, b_{s}\right)\right)=0,
$$

otherwise $h$ is given by the formula

$$
\begin{aligned}
& h\left(\left(p_{1}, \ldots, p_{r}\right) \sigma\left(b_{1}, \ldots, b_{s}\right)\right) \\
& =(-1)^{\left(\left|p_{1}\right|+1\right)\left(\left|p_{2}\right|+\cdots+\left|p_{r}\right|\right)}\left(1, \ldots, 1, p_{2}, \ldots, p_{r}\right) \sigma^{\prime}\left(b^{\prime}, b_{i+1}, \ldots, b_{s}\right),
\end{aligned}
$$

where $b^{\prime}=\mu_{\mathcal{F}(\Sigma \overline{\mathcal{P}})}\left(\Sigma p_{1} \otimes\left(b_{1}, \ldots, b_{i}\right)\right)$ with $b_{1}, \ldots, b_{i}$ the elements of $\overline{\mathcal{B}}(\mathcal{P})$ linked via at least one edge to the vertex indexed by $p_{1}$ in the graphic representative of $\left(p_{1}, \ldots, p_{r}\right) \sigma\left(b_{1}, \ldots, b_{s}\right)$. This morphism $h$ is homogenous of degree $+1(\overline{\mathcal{P}} \rightarrow \Sigma \overline{\mathcal{P}})$ and does not change the number of elements of $\overline{\mathcal{P}}$. Therefore, we have $h: E_{p, q}^{0} \rightarrow$ $E_{p, q+1}^{0}$. Intuitively, the application $h$ takes the "first" non-trivial operation between the ones of $\mathcal{P}$ on the last level, suspends it, and lifts it between the ones of $\overline{\mathcal{B}}(\mathcal{P})$. Let verify that $h d^{0}+d^{0} h=i_{d}$.

(1) The rules of signs and suspensions show that $h \delta+\delta h=0$.

(2) We have $h d_{\theta_{l}}^{\prime}+d_{\theta_{l}}^{\prime} h=i_{d}$. We first compute $d_{\theta_{l}}^{\prime}$ :

$$
\begin{aligned}
& d_{\theta_{l}}^{\prime}\left(\left(p_{1}, \ldots, p_{r}\right) \sigma\left(b_{1}, \ldots, b_{s}\right)\right) \\
& \quad=\sum(-1)^{\left|p^{\prime}\right|\left(\left|p_{j+1}\right|+\cdots+\left|p_{r}\right|+\left|b_{1}\right|+\cdots+\left|b_{k}\right|\right)} \\
& \quad \times\left(p_{1}, \ldots, p^{\prime}, p_{j+1} \ldots, p_{r}\right) \widetilde{\sigma}\left(b_{1}, \ldots, b_{k}, b_{k+1}^{\prime}, \ldots, b_{s}\right),
\end{aligned}
$$


where $b_{k+1}=\mu_{\mathcal{F}(\Sigma \overline{\mathcal{P}})}\left(\Sigma p^{\prime} \otimes b_{k+1}^{\prime}\right)$. Then, we have

$$
\begin{aligned}
h d_{\theta_{l}}^{\prime} & \left(\left(p_{1}, \ldots, p_{r}\right) \sigma\left(b_{1}, \ldots, b_{s}\right)\right) \\
& =\sum(-1)^{\left|p^{\prime}\right|\left(\left|p_{j+1}\right|+\cdots+\left|p_{r}\right|+\left|b_{1}\right|+\cdots+\left|b_{k}\right|\right)+\left(\left|p_{1}\right|+1\right)\left(\left|p_{2}\right|+\cdots+\left|p^{\prime}\right|+\cdots+\left|p_{r}\right|\right)} \\
& \times\left(1, \ldots, 1, p_{2}, \ldots, p^{\prime}, p_{j+1} \ldots, p_{r}\right) \widetilde{\sigma}^{\prime}\left(b^{\prime}, b_{i+1}, \ldots, b_{k}, b_{k+1}^{\prime}, \ldots, b_{s}\right) .
\end{aligned}
$$

The same calculus for $d_{\theta_{l}}^{\prime} h$ gives

$$
\begin{aligned}
d_{\theta_{l}}^{\prime} h & \left(\left(p_{1}, \ldots, p_{r}\right) \sigma\left(b_{1}, \ldots, b_{s}\right)\right) \\
& =\left(p_{1}, \ldots, p_{r}\right) \sigma\left(b_{1}, \ldots, b_{s}\right) \\
& -\sum(-1)^{\left|p^{\prime}\right|\left(\left|p_{j+1}\right|+\cdots+\left|p_{r}\right|+\left|b_{1}\right|+\cdots+\left|b_{k}\right|\right)+\left(\left|p_{1}\right|+1\right)\left(\left|p_{2}\right|+\cdots+\left|p^{\prime}\right|+\cdots+\left|p_{r}\right|\right)} \\
& \times\left(1, \ldots, 1, p_{2}, \ldots, p^{\prime}, p_{j+1} \ldots, p_{r}\right) \widetilde{\sigma}^{\prime}\left(b^{\prime}, b_{i+1}, \ldots, b_{k}, b_{k+1}^{\prime}, \ldots, b_{s}\right),
\end{aligned}
$$

and the sign -1 comes from the commutation of $p^{\prime}$ with $\Sigma p_{1}$.

Since $h$ is a homotopy, the chain complexes $\left(E_{p, *}^{0}, d^{0}\right)$ are acyclic for $p>0$, which means that we have

$$
E_{p, q}^{1}=0 \quad \text { for all } q \text { if } p>0
$$

We can now conclude the proof of the acyclicity of the augmented bar construction.

Theorem 4.15. The homology of the chain complex $\left(\mathcal{P} \otimes_{c} \overline{\mathcal{B}}(\mathcal{P}), d\right)$ is given by :

$$
\left\{\begin{array}{l}
H_{0}\left(\mathcal{P} \bigotimes_{c} \overline{\mathcal{B}}(\mathcal{P})\right)=I, \\
H_{n}\left(\mathcal{P} \bigotimes_{c} \overline{\mathcal{B}}(\mathcal{P})\right)=0 \quad \text { otherwise. }
\end{array}\right.
$$

Proof. Since the filtration $F_{i}$ is exhaustive $\left(\mathcal{P} \otimes_{c} \overline{\mathcal{B}}(\mathcal{P})=\bigcup_{i} F_{i}\right)$ and bounded below $\left(F_{-1}\left(\mathcal{P} \otimes_{c} \overline{\mathcal{B}}(\mathcal{P})\right)=0\right)$, we can apply the classical theorem of convergence of spectral sequences $\left(c f\right.$. W] 5.5.1). Hence, we get that the spectral sequence $E_{p, q}^{*}$ converges to the homology of $\mathcal{P} \otimes_{c} \overline{\mathcal{B}}(\mathcal{P})$ :

$$
E_{p, q}^{1} \Longrightarrow H_{p+q}\left(\mathcal{P} \otimes_{c} \overline{\mathcal{B}}(\mathcal{P}), d\right) .
$$

We conclude using Lemma 4.14 ,

Corollary 4.16. The augmentation morphism

$$
\mathcal{P} \otimes_{c} \overline{\mathcal{B}}(\mathcal{P}) \stackrel{\varepsilon_{\mathcal{P}} \bigotimes_{c} \varepsilon_{\mathcal{F}^{c}(\Sigma \overline{\mathcal{P}})}}{\longrightarrow} I \bigotimes_{c} I=I
$$

is a quasi-isomorphism.

This last result can be generalized in the following way :

We define the augmentation morphism $\varepsilon(\mathcal{P}, \mathcal{P}, \mathcal{P})$ by the composition

$\mathcal{B}(\mathcal{P}, \mathcal{P}, \mathcal{P})=\mathcal{P} \bigotimes_{c} \overline{\mathcal{B}}(\mathcal{P}) \bigotimes_{c} \mathcal{P} \stackrel{\mathcal{P} \bigotimes_{c} \varepsilon_{\mathcal{F}^{c}(\Sigma \overline{\mathcal{P}})} \bigotimes_{c} \mathcal{P}}{\longrightarrow} \mathcal{P} \bigotimes_{c} I \bigotimes_{c} \mathcal{P}=\mathcal{P} \bigotimes_{c} \mathcal{P} \rightarrow \mathcal{P} \bigotimes_{\mathcal{P}} \mathcal{P}=\mathcal{P}$.

Theorem 4.17. Let $R$ be a left differential $\mathcal{P}$-module. The augmentation morphism $\varepsilon(\mathcal{P}, \mathcal{P}, R)$

$$
\mathcal{B}(\mathcal{P}, \mathcal{P}, R)=\mathcal{P} \otimes_{\mathcal{P}} \mathcal{B}(\mathcal{P}, \mathcal{P}, \mathcal{P}) \bigotimes_{\mathcal{P}} R \stackrel{\mathcal{P} \otimes_{\mathcal{P} \varepsilon}(\mathcal{P}, \mathcal{P}, \mathcal{P}) \bigotimes_{\mathcal{P}} R}{\longrightarrow} \mathcal{P} \otimes_{\mathcal{P}} \mathcal{P} \otimes_{\mathcal{P}} R=R
$$

is a quasi-isomorphism. 
Proof. We define the same kind of filtration based on the number of elements of $\overline{\mathcal{P}}$ indexing the graphs.

Remark. Using the same arguments, we have the same results for the augmented bar construction on the right $\overline{\mathcal{B}}(\mathcal{P}) \bigotimes_{c} \mathcal{P}$.

4.3.2. Acyclicity of the augmented cobar construction. Dually, we can prove that the augmented cobar construction $\overline{\mathcal{B}}^{c}(\mathcal{C}) \otimes_{c} \mathcal{C}$ is acyclic. But in order to make the spectral sequence converge, we need to work with a weight graded coproperad $\mathcal{C}$.

Theorem 4.18. For every weight graded coaugmented coproperad $\mathcal{C}$, the homology of the augmented cobar construction $\left(\overline{\mathcal{B}}^{c}(\mathcal{C}) \bigotimes_{c} \mathcal{C}, d\right)$ is given by

$$
\left\{\begin{array}{l}
H_{0}\left(\overline{\mathcal{B}}^{c}(\mathcal{C}) \bigotimes_{c} \mathcal{C}\right)=I \\
H_{n}\left(\overline{\mathcal{B}}^{c}(\mathcal{C}) \bigotimes_{c} \mathcal{C}\right)=0 \quad \text { otherwise }
\end{array}\right.
$$

Proof. We define a filtration $F_{i}$ on $\left(\overline{\mathcal{B}}^{c}(\mathcal{C}) \bigotimes_{c} \mathcal{C}, d\right)$ by

$$
F_{i}=\bigoplus_{s \geq-i}\left(\overline{\mathcal{B}}^{c}(\mathcal{C}) \otimes_{c} \mathcal{C}\right)_{(s)} .
$$

This filtration is stable under the differential $d$ :

(1) Since the differential $\delta$ does not change the number of elements of $\overline{\mathcal{C}}$, we have $\delta\left(F_{i}\right) \subset F_{i}$.

(2) The derivation $d_{\theta^{\prime}}$ of the reduced cobar construction $\overline{\mathcal{B}}^{c}(\mathcal{C})$ splits into two parts, each element indexing a vertex. Therefore, the global number of vertices indexed by elements of $\overline{\mathcal{C}}$ is raised by 1 . The derivation $d_{\theta^{\prime}}$ verifies $d_{\theta^{\prime}}\left(F_{i}\right) \subset F_{i-1}$.

(3) The morphism $d_{\theta_{r}^{\prime}}$ decomposes elements of $\mathcal{C}$ into two parts and includes on part in $\overline{\mathcal{B}}^{c}(\mathcal{C})$. The global number of elements of $\overline{\mathcal{C}}$ indexing vertices is increasing. We have $d_{\theta_{r}^{\prime}}\left(F_{i}\right) \subset F_{i}$.

This filtration induces a spectral sequence $E_{p, q}^{*}$. Its first term is given by

$$
E_{p, q}^{0}=F_{p}\left(\left(\overline{\mathcal{B}}^{c}(\mathcal{C}) \bigotimes_{c} \mathcal{C}\right)_{p+q}\right) / F_{p-1}\left(\left(\overline{\mathcal{B}}^{c}(\mathcal{C}) \bigotimes_{c} \mathcal{C}\right)_{p+q}\right)=\left(\left(\overline{\mathcal{B}}^{c}(\mathcal{C}) \bigotimes_{c} \mathcal{C}\right)_{(-p)}\right)_{p+q}
$$

The differential $d^{0}$ is the sum of two terms $d^{0}=\delta+d_{\theta_{r}^{\prime}}^{\prime}$, where $d_{\theta_{r}^{\prime}}^{\prime}$ corresponds to $d_{\theta_{r}^{\prime}}$ when it does not strictly increase the number of elements of $\overline{\mathcal{C}}$. The morphism $d_{\theta_{r}^{\prime}}^{\prime}$ takes elements of $\overline{\mathcal{C}}$ in the first level, desuspends them and includes them in $\overline{\mathcal{B}}^{c}(\mathcal{C})$.

We then show that

$$
E_{p, q}^{1}= \begin{cases}I & \text { if } p=q=0 \\ 0 & \text { otherwise }\end{cases}
$$

We define the image under the homotopy $h$ of an element $\left(b_{1}, \ldots, b_{r}\right) \sigma\left(c_{1}, \ldots\right.$, $\left.c_{s}\right)$ of $\overline{\mathcal{B}}^{c}(\mathcal{C}) \otimes \mathcal{C}$ by first decomposing $b_{1} \in \overline{\mathcal{B}}^{c}(\mathcal{C})_{(n)}$ :

$$
b_{1} \longmapsto \sum b_{1}^{\prime} \otimes \Sigma^{-1} c,
$$

where $b_{1}^{\prime} \in \overline{\mathcal{B}}^{c}(\mathcal{C})_{(n-1)}$ and $c \in \overline{\mathcal{C}}$. If the element $\Sigma^{-1} c$ is linked above to elements of $I$, then we suspend it and include it in the line of elements of $\mathcal{C}$. Using the same arguments, one can show that $h d^{0}+d^{0} h=i_{d}$. 
Since the coproperad $\mathcal{C}$ is weight graded, one can decompose the chain complex $\overline{\mathcal{B}}^{c}(\mathcal{C}) \otimes_{c} \mathcal{C}$ into a direct sum indexed by the total weight :

$$
\overline{\mathcal{B}}^{c}(\mathcal{C}) \bigotimes_{c} \mathcal{C}=\bigoplus_{\rho \in \mathbb{N}}\left(\overline{\mathcal{B}}^{c}(\mathcal{C}) \otimes \mathcal{C}\right)^{(\rho)} .
$$

This decomposition is compatible with the filtration $F_{i}$ and with the homotopy $h$. Therefore, we have

$$
\begin{gathered}
E_{p, q}^{1}\left(\left(\overline{\mathcal{B}}^{c}(\mathcal{C}) \bigotimes_{c} \mathcal{C}\right)^{(\rho)}\right)=0 \text { for every } p, q \text { when } \rho>0 \text { and } \\
E_{p, q}^{1}\left(\left(\overline{\mathcal{B}}^{c}(\mathcal{C}) \bigotimes_{c} \mathcal{C}\right)^{(0)}\right)= \begin{cases}I & \text { if } p=q=0, \\
0 & \text { otherwise. }\end{cases}
\end{gathered}
$$

Since the filtration $F_{i}$ is bounded on the sub-complex $\left(\overline{\mathcal{B}}^{c}(\mathcal{C}) \bigotimes_{c} \mathcal{C}\right)^{(\rho)}$,

$$
F_{0}\left(\left(\overline{\mathcal{B}}^{c}(\mathcal{C}) \bigotimes_{\mathcal{C}} \mathcal{C}\right)^{(\rho)}\right)=\left(\overline{\mathcal{B}}^{c}(\mathcal{C}) \bigotimes_{\mathcal{C}} \mathcal{C}\right)^{(\rho)} \quad \text { and } \quad F_{-\rho-1}\left(\left(\overline{\mathcal{B}}^{c}(\mathcal{C}) \bigotimes_{\mathcal{C}} \mathcal{C}\right)^{(\rho)}\right)=0,
$$

we can apply the classical theorem of convergence of spectral sequences $(c f .[\mathrm{W}]$ 5.5.1). This gives that the spectral sequence $E_{p, q}^{*}\left(\left(\overline{\mathcal{B}}^{c}(\mathcal{C}) \bigotimes_{c} \mathcal{C}\right)^{(\rho)}\right)$ converges to the homology of $\left(\overline{\mathcal{B}}^{c}(\mathcal{C}) \bigotimes_{c} \mathcal{C}\right)^{(\rho)}$. We conclude by taking the direct sum on $\rho$.

Remark. Using the same methods, we can show that the augmented bar construction on the left is acyclic.

\section{COMPARISON LEMMAS}

In this section we prove comparison lemmas for quasi-free $\mathcal{P}$-modules and quasifree properads. These lemmas are generalizations to the composition product $\nabla_{c}$ of classical lemmas for the tensor product $\otimes_{k}$. Notice that B. Fresse has given in $\mathrm{Fr}$ such lemmas for the composition product $\circ$ of operads. These technical lemmas allow us to show that the bar-cobar construction of a weight graded properad $\mathcal{P}$ is a resolution of $\mathcal{P}$.

\subsection{Comparison lemma for quasi-free modules.}

5.1.1. Filtration and spectral sequence of an analytic quasi-free module. Let $\mathcal{P}$ be a weight graded differential properad $\mathcal{P}$ and let $L=M \nabla_{c} \mathcal{P}$ be an analytic quasi-free $\mathcal{P}$-module on the right, where $M=\Upsilon(V)$. Denote $M_{d}^{(\alpha)}$ to be the sub$\mathbb{S}$-bimodule of $M$ composed by elements of homological degree $d$ and global weight $\alpha$ (cf. 3.4).

We define the following filtration on $L$ :

$$
F_{s}(L):=\bigoplus_{\Xi} \bigoplus_{|\bar{d}|+|\bar{\alpha}| \leq s} M_{\bar{d}}^{(\bar{\alpha})}(\bar{l}, \bar{k}) \otimes_{\mathbb{S}_{\bar{k}}} k\left[\mathbb{S}_{\bar{k}, \bar{\jmath}}^{c}\right] \otimes_{\mathbb{S}_{\bar{\jmath}}} \mathcal{P}_{\bar{e}}(\bar{\jmath}, \bar{\imath})
$$

where the direct sum $(\Xi)$ is over the integers $m, n$ and $N$ and the tuples $\bar{l}, \bar{k}, \bar{\jmath}, \bar{\imath}$.

Lemma 5.1. The filtration $F_{s}$ is stable under the differential $d$ of $L$.

Proof. The differential $d$ is the sum of three terms.

- The differential $\delta_{M}$, induced by the one of M, decreases the homological degree $|\bar{d}|$ by 1 . Therefore, we have $\delta_{M}\left(F_{s}\right) \subset F_{s-1}$.

- The differential $\delta_{\mathcal{P}}$, induced by the one of $\mathcal{P}$, decreases the homological degree $|\bar{e}|$ by 1 . We have $\delta_{\mathcal{P}}\left(F_{s}\right) \subset F_{s}$. 
- By definition of the analytic quasi-free $\mathcal{P}$-modules, one has that the morphism $d_{\theta}$ decreases the total graduation $|\bar{\alpha}|$ by at least 1 and the homological degree $|\bar{d}|$ by 1 . We have $d_{\theta}\left(F_{s}\right) \subset F_{s-2}$.

This filtration gives a spectral sequence $E_{s, t}^{*}$. The first term of this spectral sequence $E_{s, t}^{0}$ is isomorphic to the following dg-S-bimodule :

$$
E_{s, t}^{0}=\bigoplus_{\Xi} \bigoplus_{r=0}^{s} \bigoplus_{\substack{|\bar{d}|=s-r,|\bar{e}|=t+r \\|\bar{\alpha}|=r}} M_{\bar{d}}^{(\bar{\alpha})}(\bar{l}, \bar{k}) \otimes_{\mathbb{S}_{\bar{k}}} k\left[\mathbb{S}_{\bar{k}, \bar{\jmath}}^{c}\right] \otimes_{\mathbb{S}_{\bar{\jmath}}} \mathcal{P}_{\bar{e}}(\bar{\jmath}, \bar{l}) .
$$

Using the same notations as in section 3.2 , we have

$$
E_{s, t}^{0}=\bigoplus_{r=0}^{s} I_{s-r, t+r}^{0}\left(\bigoplus_{|\bar{\alpha}|=r} M^{(\bar{\alpha})} \bigotimes_{c} \mathcal{P}\right) .
$$

On can decompose the weight graded differential $\mathbb{S}$-bimodule $L$ with its weight $L=\bigoplus_{\rho} L^{(\rho)}$. Since this decomposition is stable under the filtration $F_{s}$, the spectral sequence $E_{s, t}^{*}$ is isomorphic to

$$
E_{s, t}^{*}(L)=\bigoplus_{\rho \in \mathbb{N}} E_{s, t}^{*}\left(L^{(\rho)}\right) .
$$

By definition of the analytic quasi-free $\mathcal{P}$-modules, the weight of the elements of $V$ is at least 1 . Hence, we have

$$
E_{s, t}^{0}\left(L^{(\rho)}\right)=\bigoplus_{r=0}^{\min (s, \rho)} I_{s-r, t+r}^{0}\left(\left(\bigoplus_{|\bar{\alpha}|=r} M^{(\bar{\alpha})} \otimes_{c} \mathcal{P}\right) \bigcap L^{(\rho)}\right) .
$$

Proposition 5.2. Let $L$ be an analytic quasi-free $\mathcal{P}$-module. The spectral sequence $E_{s, t}^{*}$ converges to the homology of $L$ :

$$
E_{s, t}^{*} \Longrightarrow H_{s+t}(L, d) \text {. }
$$

The differential $d^{0}$ on $E_{s, t}^{0}$ is given by $\delta_{\mathcal{P}}$, and the differential $d^{1}$ on $E_{s, t}^{1}$ is given by $\delta_{M}$. Therefore, we have

$$
E_{s, t}^{2}=\bigoplus_{r=0}^{s} I_{s-r, t+r}^{2}\left(\bigoplus_{|\bar{\alpha}|=r} M^{(\bar{\alpha})} \otimes_{c} \mathcal{P}\right),
$$

which can be written

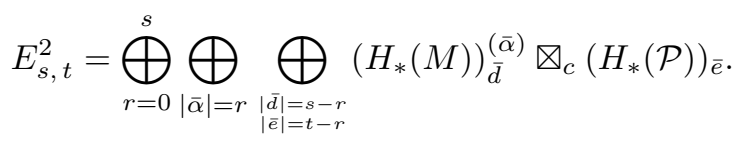

Proof. Since the filtration is exhaustive $\bigcup_{s} F_{s}=L$ and bounded below $F_{-1}=0$, the classical theorem of convergence of spectral sequences (cf. [W] 5.5.1) shows that $E_{s, t}^{*}$ converges to the homology of $L$.

The form of the differentials $d^{0}$ and $d^{1}$ comes from the study of the action of $d=\delta_{M}+\delta_{\mathcal{P}}+d_{\theta}$ on the filtration $F_{s}$. Also, the formula of $E_{s, t}^{2}$ comes from Proposition 3.4 . 
5.1.2. Comparison lemma for analytic quasi-free modules. To show the comparison lemma, we need to prove the following lemma first.

Lemma 5.3. Let $\Psi: \mathcal{P} \rightarrow \mathcal{P}^{\prime}$ be a morphism of weight graded connected $d g$ properads. Let $(L, \lambda)$ be an analytic quasi-free $\mathcal{P}$-module and $\left(L^{\prime}, \lambda^{\prime}\right)$ be an analytic quasi-free $\mathcal{P}^{\prime}$-module. Denote $\bar{L}=M$ and $\bar{L}^{\prime}=M^{\prime}$ as the indecomposable quotients. We consider the action of $\mathcal{P}$ on $L^{\prime}$ induced by the morphism $\Psi$. Let $\Phi: L \rightarrow L^{\prime}$ be a morphism of weight graded differential $\mathcal{P}$-modules:

(1) The morphism $\Phi$ preserves the filtration $F_{s}$ and leads to a morphism of spectral sequences

$$
E^{*}(\Phi): E^{*}(L) \rightarrow E^{*}\left(L^{\prime}\right)
$$

(2) Let $\bar{\Phi}: M \rightarrow M^{\prime}$ be the morphism of $d g-\mathbb{S}$-bimodules induced by $\Phi$. The morphism $E^{0}(\Phi): M \otimes_{c} \mathcal{P} \rightarrow M^{\prime} \bigotimes_{c} \mathcal{P}^{\prime}$ is equal to $E^{0}=\bar{\Phi} \bigotimes_{c} \Psi$.

Proof.

(1) Let $\left(m_{1}, \ldots, m_{b}\right) \sigma\left(p_{1}, \ldots, p_{a}\right)$ be an element of $F_{s}\left(M \otimes_{c} \mathcal{P}\right)$. It means that $|\bar{d}|+|\bar{\alpha}| \leq s$. Since, $\Phi$ is a morphism of $\mathcal{P}$-modules, we have

$$
\begin{aligned}
& \Phi\left(\left(m_{1}, \ldots, m_{b}\right) \sigma\left(p_{1}, \ldots, p_{a}\right)\right)=\Phi \circ \lambda\left(\left(m_{1}, \ldots, m_{b}\right) \sigma\left(p_{1}, \ldots, p_{a}\right)\right) \\
& =\lambda^{\prime}\left(\left(\Phi\left(m_{1}\right), \ldots, \Phi\left(m_{b}\right)\right) \sigma\left(\Psi\left(p_{1}\right), \ldots, \Psi\left(p_{a}\right)\right)\right) .
\end{aligned}
$$

Denote

$$
\Phi\left(m_{i}\right)=\left(m_{1}^{i}, \ldots, m_{b_{i}}^{i}\right) \sigma^{i}\left(p_{1}^{i}, \ldots, p_{a_{i}}^{i}\right) .
$$

The application $\Phi$ is a morphism of dg- $\mathbb{S}$-bimodules. Therefore, we have $d_{i}=\left|\bar{d}^{i}\right|+\left|\overline{e^{i}}\right|$ which implies $\left|\bar{d}^{i}\right| \leq d_{i}$ and $\sum_{i}\left|\bar{d}^{i}\right| \leq|\bar{d}|$. Since $\Phi$ is a morphism of weight graded $\mathcal{P}$-modules, we have $\sum_{i}\left|\bar{\alpha}^{i}\right| \leq|\bar{\alpha}|$. We conclude that

$$
\Phi\left(\left(m_{1}, \ldots, m_{b}\right) \sigma\left(p_{1}, \ldots, p_{a}\right)\right) \in F_{s}\left(L^{\prime}\right) .
$$

(2) The morphism $E_{s, t}^{0}(\Phi)$ is given by the following morphism on the quotients:

$$
E_{s, t}^{0}: F_{s}\left(L_{s+t}\right) / F_{s-1}\left(L_{s+t}\right) \rightarrow F_{s}\left(L_{s+t}^{\prime}\right) / F_{s-1}\left(L_{s+t}^{\prime}\right) .
$$

Let $\left(m_{1}, \ldots, m_{b}\right) \sigma\left(p_{1}, \ldots, p_{a}\right)$ be an element of $E_{s, t}^{0}$, where $|\bar{\alpha}|=r \leq s$, $|\bar{d}|=s-r$ and $|\bar{e}|=t+r$. We have

$$
E_{s, t}^{0}(\Phi)\left(\left(m_{1}, \ldots, m_{b}\right) \sigma\left(p_{1}, \ldots, p_{a}\right)\right)=0
$$

if and only if $\Phi\left(\left(m_{1}, \ldots, m_{b}\right) \sigma\left(p_{1}, \ldots, p_{a}\right)\right) \in F_{s-1}\left(L^{\prime}\right)$. We have seen that

$\Phi\left(\left(m_{1}, \ldots, m_{b}\right) \sigma\left(p_{1}, \ldots, p_{a}\right)\right)=\lambda^{\prime}\left(\left(\Phi\left(m_{1}\right), \ldots, \Phi\left(m_{b}\right)\right) \sigma\left(\Psi\left(p_{1}\right), \ldots, \Psi\left(p_{a}\right)\right)\right)$.

Therefore $\Phi\left(\left(m_{1}, \ldots, m_{b}\right) \sigma\left(p_{1}, \ldots, p_{a}\right)\right)$ belongs to $F_{s}\left(L_{s+t}^{\prime}\right) \backslash F_{s-1}\left(L_{s+t}^{\prime}\right)$ if and only if $\Phi\left(m_{i}\right)=m_{i}=\bar{\Phi}\left(m_{i}\right)$. Consider $\Phi\left(m_{i}\right)=\left(m_{1}^{i}, \ldots, m_{b_{i}}^{i}\right)$ $\sigma^{i}\left(p_{1}^{i}, \ldots, p_{a_{i}}^{i}\right)$ where at least one $p_{j}^{i}$ does not belong to $I$. By definition of a weight graded properad, the weight of these $p_{j}^{i}$ is at least 1 . Since $\Phi$ preserves the total weight, we have $\left|\bar{\alpha}^{i}\right|<\alpha^{i}$. Finally, the morphism $E^{0}(\Phi)$ is equal to $\bar{\Phi} \bigotimes_{c} \Psi$. 
Theorem 5.4 (Comparison lemma for analytic quasi-free modules). With the same notations as in the previous lemma, if two of the following morphisms

$$
\begin{cases}\Psi & : \mathcal{P} \rightarrow \mathcal{P}^{\prime} \\ \bar{\Phi} & : M \rightarrow M^{\prime} \\ \Phi & : L \rightarrow L^{\prime}\end{cases}
$$

are quasi-isomorphisms, then the third one is also a quasi-isomorphism.

Remark. If the properads are not weight graded and if $\Phi=\bar{\Phi} \otimes_{c} \Psi$, when $\bar{\Phi}$ and $\Psi$ are quasi-isomorphisms, then $\Phi$ is also a quasi-isomorphism. The proof is straight. We have immediately $E^{0}(\Phi)=\bar{\Phi} \otimes_{c} \Psi$. Since $\bar{\Phi}$ and $\Psi$ are quasi-isomorphisms, we get that $E^{2}(\Phi)$ is an isomorphism by Proposition $5.2\left(d^{0}=\delta_{\mathcal{P}}\right.$ et $\left.d^{1}=\delta_{M}\right)$. Also, with the same proposition, the convergence of the spectral sequence $E^{*}$ shows that $\Phi$ is a quasi-isomorphism between $L$ and $L^{\prime}$.

Proof.

(1) Suppose that $\Psi: \mathcal{P} \rightarrow \mathcal{P}^{\prime}$ and $\bar{\Phi}: M \rightarrow M^{\prime}$ are quasi-isomorphisms. The previous lemma shows that $\Phi$ induces a morphism of spectral sequences $E^{*}(\Phi): E^{*}(L) \rightarrow E^{*}\left(L^{\prime}\right)$ and that $E^{0}(\Phi)=\bar{\Phi} \otimes_{c} \Psi$. We conclude with the same arguments.

(2) Suppose that $\Psi: \mathcal{P} \rightarrow \mathcal{P}^{\prime}$ and $\Phi: L \rightarrow L^{\prime}$ are quasi-isomorphisms. We have seen that the spectral sequence $E_{s, t}^{*}$ preserves the decomposition $L^{(\rho)}$. Moreover, we have

$$
E_{s, t}^{2}\left(L^{(\rho)}\right)=\bigoplus_{\Xi} \bigoplus_{r=\max (0,-t)}^{\min (s, \rho)} I_{s-r, t+r}^{2}\left(\bigoplus_{\chi} M_{\bar{d}}^{(\bar{\alpha})}(\bar{l}, \bar{k}) \otimes_{\mathbb{S}_{\bar{k}}} k\left[\mathbb{S}_{\bar{k}, \bar{\jmath}}^{c}\right] \otimes_{\mathbb{S}_{\bar{\jmath}}} \mathcal{P}_{\bar{e}}^{(\bar{\beta})}(\bar{\jmath}, \bar{l})\right),
$$

where the second sum $(\chi)$ is on $|\bar{\alpha}|=r,|\bar{\beta}|=\rho-r,|\bar{d}|=s-r$ and $|\bar{e}|=t+r$. When $s \geq \rho$, we have for $r=\rho$

$$
\begin{gathered}
I_{s-\rho, t+\rho}^{2}\left(\bigoplus_{\chi} M_{\bar{d}}^{(\bar{\alpha})}(\bar{l}, \bar{k}) \otimes_{\mathbb{S}_{\bar{k}}} k\left[\mathbb{S}_{\bar{k}, \bar{\jmath}}^{c}\right] \otimes_{\mathbb{S}_{\bar{\jmath}}} \mathcal{P}_{\bar{e}}^{(\bar{\beta})}(\bar{\jmath}, \bar{\imath})\right) \\
=I_{s-\rho, t+\rho}^{2}\left(\bigoplus_{\substack{|\bar{\alpha}|=\rho \\
|\bar{d}|=s-\rho}} M_{\overline{\bar{d}}}^{(\bar{\alpha})}\right) \\
= \begin{cases}H_{s-\rho}\left(M^{(\rho)}\right) & \text { if } t=-\rho, \\
0 & \text { otherwise. }\end{cases}
\end{gathered}
$$

Finally, we have

$$
\begin{cases}E_{s, t}^{2}\left(L^{(\rho)}\right)=0 & \text { if } t<-\rho \\ E_{s,-\rho}^{2}\left(L^{(\rho)}\right)=H_{s-\rho}\left(M^{(\rho)}\right) & \text { if } s \geq \rho\end{cases}
$$

We now show by induction on $\rho$ that $\bar{\Phi}^{(\rho)}: M^{(\rho)} \rightarrow M^{\prime(\rho)}$ is a quasiisomorphism $H_{*}\left(\bar{\Phi}^{(\rho)}\right): H_{*}\left(M^{(\rho)}\right) \rightarrow H_{*}\left(M^{\prime(\rho)}\right)$.

For $\rho=0$, since $L^{(0)}=M^{(0)}$, we have $\bar{\Phi}^{(0)}=\Phi^{(0)}$, which is a quasiisomorphism.

Suppose now that the result is true for $r<\rho$ in every (homological) degree $*$ and for $r=\rho$ in degree $*<d$. Let us show that it is also true for 
$*=d$. (Since every chain complex is concentrated in non-negative degree, we have that $H_{s}\left(\bar{\Phi}^{(\rho)}\right)$ is always an isomorphism for $s<0$.)

By the preceding lemma, we have

$$
E_{s, t}^{2}\left(\Phi^{(\rho)}\right)=\bigoplus_{r=0}^{\min (s, \rho)} I_{s-r, t+r}^{2}\left(\bigoplus_{|\bar{\alpha}|=r} \bar{\Phi}^{(\bar{\alpha})} \bigotimes_{c} \Psi\right) .
$$

With the induction hypothesis, we get that $E_{s, t}^{2}\left(\Phi^{(\rho)}\right): E_{s, t}^{2}\left(L^{(\rho)}\right) \rightarrow$ $E_{s, t}^{2}\left(L^{\prime(\rho)}\right)$ is an isomorphism for $s<d+\rho$. Let us show that

$$
E_{d+\rho,-\rho}^{2}\left(\Phi^{(\rho)}\right): E_{d+\rho,-\rho}^{2}\left(L^{(\rho)}\right) \rightarrow E_{d+\rho,-\rho}^{2}\left(L^{\prime(\rho)}\right)
$$

is still an isomorphism. To do that, we consider the mapping cone of $\Phi^{(\rho)}: \operatorname{cone}\left(\Phi^{(\rho)}\right)=\Sigma^{-1} L^{(\rho)} \oplus L^{\prime(\rho)}$. On this cone, we define the following filtration:

$$
F_{s}\left(\operatorname{cone}\left(\Phi^{(\rho)}\right)\right):=F_{s-1}\left(\Sigma^{-1} L^{(\rho)}\right) \oplus F_{s}\left(L^{\prime(\rho)}\right) .
$$

This filtration induces a spectral sequence which verifies

$$
E_{*, t}^{1}\left(\operatorname{cone}\left(\Phi^{(\rho)}\right)\right)=\operatorname{cone}\left(E_{*, t}^{1}\left(\Phi^{(\rho)}\right)\right) .
$$

The mapping cone of $E_{*, t}^{1}\left(\Phi^{(\rho)}\right)$ verifies the long exact sequence

$$
\begin{aligned}
& \cdots \rightarrow H_{s+1}\left(\operatorname{cone}\left(E_{*, t}^{1}\left(\Phi^{(\rho)}\right)\right)\right) \rightarrow H_{s}\left(E_{*, t}^{1}\left(L^{(\rho)}\right)\right) \\
& \stackrel{H_{s}\left(E_{*, t}^{1}\left(\Phi^{(\rho)}\right)\right)}{\longrightarrow} H_{s}\left(E_{*, t}^{1}\left(L^{\prime(\rho)}\right)\right) \rightarrow H_{s}\left(\operatorname{cone}\left(E_{*, t}^{1}\left(\Phi^{(\rho)}\right)\right)\right) \\
& \rightarrow H_{s-1}\left(E_{*, t}^{1}\left(L^{(\rho)}\right)\right) \rightarrow \cdots
\end{aligned}
$$

which finally gives the following long exact sequence $(\xi)$ :

$$
\begin{gathered}
\cdots \longrightarrow E_{s+1, t}^{2}\left(\operatorname{cone}\left(\Phi^{(\rho)}\right)\right) \longrightarrow E_{s, t}^{2}\left(L^{(\rho)}\right) \stackrel{E_{s, t}^{2}\left(\Phi^{(\rho)}\right)}{\longrightarrow} E_{s, t}^{2}\left(L^{\prime(\rho)}\right) \\
\longrightarrow E_{s, t}^{2}\left(\operatorname{cone}\left(\Phi^{(\rho)}\right)\right) \longrightarrow E_{s-1, t}^{2}\left(L^{(\rho)}\right) \longrightarrow
\end{gathered}
$$

We have seen that for all $t<-\rho$,

$$
E_{s, t}^{2}\left(L^{(\rho)}\right)=E_{s, t}^{2}\left(L^{\prime(\rho)}\right)=0 .
$$

Therefore, the long exact sequence $(\xi)$ shows that $E_{s, t}^{2}\left(\operatorname{cone}\left(\Phi^{(\rho)}\right)\right)=0$ for all $s$, when $t<-\rho$.

In the same way, we have seen that $E_{s, t}^{2}\left(\Phi^{(\rho)}\right)$ is an isomorphism for $s<$ $d+\rho$. Thanks to the long exact sequence $(\xi)$, we get that $E_{s, t}^{2}\left(\operatorname{cone}\left(\Phi^{(\rho)}\right)\right)=$ 0 for all $t$, when $s<d+\rho$.

These two results prove that

$$
\left\{\begin{array}{l}
E_{d+\rho,-\rho}^{2}\left(\operatorname{cone}\left(\Phi^{(\rho)}\right)\right)=E_{d+\rho,-\rho}^{\infty}\left(\operatorname{cone}\left(\Phi^{(\rho)}\right)\right) \\
E_{d+\rho+1,-\rho}^{2}\left(\operatorname{cone}\left(\Phi^{(\rho)}\right)\right)=E_{d+\rho+1,-\rho}^{\infty}\left(\operatorname{cone}\left(\Phi^{(\rho)}\right)\right) .
\end{array}\right.
$$

Since the filtration $F_{s}$ of the mapping cone $\Phi^{(\rho)}$ is bounded below and exhaustive, we know that the spectral sequence $E_{s, t}^{*}\left(\operatorname{cone}\left(\Phi^{(\rho)}\right)\right)$ converges 
to the homology of this mapping cone. The morphism $\Phi^{(\rho)}$ is a quasiisomorphism. Hence this homology is null and we have the equalities :

$$
\left\{\begin{array}{l}
E_{d+\rho,-\rho}^{2}\left(\operatorname{cone}\left(\Phi^{(\rho)}\right)\right)=E_{d+\rho,-\rho}^{\infty}\left(\operatorname{cone}\left(\Phi^{(\rho)}\right)\right)=0, \\
E_{d+\rho+1,-\rho}^{2}\left(\operatorname{cone}\left(\Phi^{(\rho)}\right)\right)=E_{d+\rho+1,-\rho}^{\infty}\left(\operatorname{cone}\left(\Phi^{(\rho)}\right)\right)=0 .
\end{array}\right.
$$

If we inject these equalities in the long exact sequence $(\xi)$, we show that $E_{d+\rho,-\rho}^{2}\left(\Phi^{(\rho)}\right)$ is an isomorphism.

We conclude using the fact that $E_{d+\rho,-\rho}^{2}\left(L^{(\rho)}\right)=H_{d}\left(M^{(\rho)}\right)$ and that $E_{d+\rho,-\rho}^{2}\left(L^{\prime(\rho)}\right)=H_{d}\left(M^{\prime(\rho)}\right)$. Therefore, $E_{d+\rho,-\rho}^{2}\left(\Phi^{(\rho)}\right)$ is equal to $H_{d}\left(\bar{\Phi}^{(\rho)}\right)$ : $H_{d}\left(M^{(\rho)}\right) \rightarrow H_{d}\left(M^{\prime(\rho)}\right)$ which is an isomorphism.

(3) Suppose that $\Phi: L \rightarrow L^{\prime}$ and $\bar{\Phi}: M \rightarrow M^{\prime}$ are quasi-isomorphisms. We are going to use the same methods. Since $M^{(0)}=I$, we get from the relation

$$
E_{s, t}^{2}\left(L^{(\rho)}\right)=\bigoplus_{\Xi} \bigoplus_{r=\max (0,-t)}^{\min (s, \rho)} I_{s-r, t+r}^{2}\left(\bigoplus_{\chi} M_{\bar{d}}^{(\bar{\alpha})}(\bar{l}, \bar{k}) \otimes_{\mathbb{S}_{\bar{k}}} k\left[\mathbb{S}_{\bar{k}, \bar{\jmath}}^{c}\right] \otimes_{\mathbb{S}_{\bar{\jmath}}} \mathcal{P}_{\bar{e}}^{(\bar{\beta})}(\bar{\jmath}, \bar{\imath})\right),
$$

where the second direct sum $\chi$ is on $|\bar{\alpha}|=r,|\bar{\beta}|=\rho-r,|\bar{d}|=s-r$ and $|\bar{e}|=t+r$, that for $s=0$

$$
E_{0, t}^{2}\left(L^{(\rho)}\right)=I_{0, t}^{2}\left(\mathcal{P}_{t}^{(\rho)}\right)=H_{t}\left(\mathcal{P}^{(\rho)}\right) .
$$

One can see that

$$
E_{s, t}^{2}\left(L^{(\rho)}\right)=0
$$

for $s<0$.

Let us show that the morphisms $\Psi^{(\rho)}: \mathcal{P}^{(\rho)} \rightarrow \mathcal{P}^{\prime(\rho)}$ are quasi-isomorphisms, by induction on $\rho$.

By definition of connected properads, we have that $\mathcal{P}^{(0)}=\mathcal{P}^{(0)}=I$. Hence, the morphism $\Psi^{(0)}=i d_{I}$ is a quasi-isomorphism.

Suppose that the result is true for every $r<\rho$. We show by induction on $t$ that

$$
H_{t}\left(\Psi^{(\rho)}\right): H_{t}\left(\mathcal{P}^{(\rho)}\right) \rightarrow H_{t}\left(\mathcal{P}^{\prime(\rho)}\right)
$$

is an isomorphism. We know that it is true for $t<0$. Suppose that it is still true for $t<e$. By the previous lemma, we have

$$
E_{s, t}^{2}\left(\Phi^{(\rho)}\right)=\bigoplus_{r=0}^{\min (s, \rho)} I_{s-r, t+r}^{2}\left(\bigoplus_{\substack{|\bar{\alpha}|=r \\|\bar{\beta}|=\rho-r}} \bar{\Phi}^{(\bar{\alpha})} \bigotimes_{c} \Psi^{(\bar{\beta})}\right) .
$$

By the induction hypothesis, we have that $E_{s, t}^{2}\left(\Phi^{(\rho)}\right): E_{s, t}^{2}\left(L^{(\rho)}\right) \rightarrow$ $E_{s, t}^{2}\left(L^{\prime(\rho)}\right)$ is an isomorphism for every $s$, when $t<e$. By injecting this in the long exact sequence $(\xi)$, we show that $E_{s, t}^{2}\left(\operatorname{cone}\left(\Phi^{(\rho)}\right)\right)=0$ for all $s$, when $t<e$.

The form of the filtration $F_{s}\left(\operatorname{cone}\left(\Phi^{(\rho)}\right)\right)=F_{s-1}\left(\Sigma^{-1} L^{(\rho)}\right) \oplus F_{s}\left(L^{\prime(\rho)}\right)$ and the fact that $F_{-1}\left(L^{(\rho)}\right)=0$ show that $E_{s, t}^{2}\left(\operatorname{cone}\left(\Phi^{(\rho)}\right)\right)=0$ for $s<0$.

These two results with the convergence of the spectral sequence $E_{s, t}^{*}\left(\operatorname{cone}\left(\Phi^{(\rho)}\right)\right)$ to the homology of the cone of $\Phi^{(\rho)}$, which is null, show that

$$
\left\{\begin{array}{l}
E_{0, e}^{2}\left(\operatorname{cone}\left(\Phi^{(\rho)}\right)\right)=E_{0, e}^{\infty}\left(\operatorname{cone}\left(\Phi^{(\rho)}\right)\right)=0 \\
E_{1, e}^{2}\left(\operatorname{cone}\left(\Phi^{(\rho)}\right)\right)=E_{1, e}^{\infty}\left(\operatorname{cone}\left(\Phi^{(\rho)}\right)\right)=0 .
\end{array}\right.
$$


When we inject these two equalities in the long exact sequence $(\xi)$, we get that the morphism

$$
E_{0, e}^{2}\left(L^{(\rho)}\right) \stackrel{E_{0, e}^{2}\left(^{(\rho)}\right)}{\longrightarrow} E_{0, e}^{2}\left(L^{\prime(\rho)}\right)
$$

is an isomorphism. We conclude using the equalities $E_{0, e}^{2}\left(L^{(\rho)}\right)=H_{e}\left(\mathcal{P}^{(\rho)}\right)$, $E_{0, e}^{2}\left(L^{\prime(\rho)}\right)=H_{e}\left(\mathcal{P}^{\prime(\rho)}\right)$ and $E_{0, e}^{2}\left(\Phi^{(\rho)}\right)=H_{e}\left(\Psi^{(\rho)}\right)$.

5.1.3. Comparison lemma for analytic quasi-cofree comodules. We can prove the same kind of result for analytic quasi-cofree comodules. Let $\mathcal{C}$ be a connected dg-coproperad and let $L=M \otimes_{c} \mathcal{C}$ be an analytic quasi-cofree $\mathcal{C}$-comodule on the right, where $M=\Upsilon(V)$. On this chain complex, we define the following filtration:

$$
F_{s}^{\prime}(L):=\bigoplus_{(\Xi)} \bigoplus_{|\bar{e}|+|\bar{\beta}| \leq s} M_{\bar{d}}(\bar{l}, \bar{k}) \otimes_{\mathbb{S}_{\bar{k}}} k\left[\mathbb{S}_{\bar{k}, \bar{\jmath}}^{c}\right] \otimes_{\mathbb{S}_{\bar{\jmath}}} \mathcal{C}_{\bar{e}}^{(\bar{\beta})}(\bar{\jmath}, \bar{\imath}),
$$

where the direct sum $(\Xi)$ is on the integers $m, n$ and $N$ and on the tuples $\bar{l}, \bar{k}, \bar{\jmath}$, $\bar{\imath}, \bar{d}, \bar{e}$ and $\bar{\beta}$ such that $|\bar{e}|+|\bar{\beta}| \leq s$.

Applying the same arguments to this filtration and the associated spectral sequence, we get the following theorem.

Theorem 5.5 (Comparison lemma for analytic quasi-cofree comodules). Let $\Psi$ : $\mathcal{C} \rightarrow \mathcal{C}^{\prime}$ be a morphism of the weight graded connected dg-coproperad and let $(L, \lambda)$ and $\left(L^{\prime}, \lambda^{\prime}\right)$ be two analytic quasi-cofree comodules on $\mathcal{C}$ and $\mathcal{C}^{\prime}$. Denote $\bar{L}=M$ and $\bar{L}^{\prime}=M^{\prime}$ as the indecomposable quotients. Let $\Phi: L \rightarrow L^{\prime}$ be a morphism of analytic $\mathcal{C}^{\prime}$-comodules, where the structure of $\mathcal{C}^{\prime}$-comodule on $L$ is given by the functor $\Psi$. Denote $\bar{\Phi}: M \rightarrow M^{\prime}$ as the morphism of $d g-\mathbb{S}$-bimodules induced by $\Phi$.

If two of the three morphisms $\left\{\begin{array}{l}\Psi: \mathcal{C} \rightarrow \mathcal{C}^{\prime}, \\ \Phi: M \rightarrow M, \\ \Phi: L \rightarrow L^{\prime},\end{array}\right.$ the third one is a quasi-isomorphism.

5.1.4. Bar-cobar resolution. In this section, we use the two last theorems to prove that the bar-cobar construction on a weight graded properad $\mathcal{P}$ is a resolution of $\mathcal{P}$. Notice that this result is a generalization to properads of one given by $\mathrm{V}$. Ginzburg and M.M. Kapranov for operads in GK].

We define the morphism $\zeta: \overline{\mathcal{B}}^{c}(\overline{\mathcal{B}}(\mathcal{P})) \rightarrow \mathcal{P}$ by the following composition:

$$
\overline{\mathcal{B}}^{c}(\overline{\mathcal{B}}(\mathcal{P}))=\mathcal{F}\left(\Sigma^{-1} \overline{\mathcal{F}}^{c}(\Sigma \overline{\mathcal{P}})\right) \longrightarrow \mathcal{F}\left(\Sigma^{-1} \overline{\mathcal{F}}_{(1)}^{c}(\Sigma \overline{\mathcal{P}})\right)=\mathcal{F}(\overline{\mathcal{P}}) \stackrel{c_{\mathcal{P}}}{\longrightarrow} \mathcal{P},
$$

where the morphism $c_{\mathcal{P}}$ corresponds to the compositions $\mu$ of all the elements of $\mathcal{P}$ that are indexing a graph of $\mathcal{F}(\overline{\mathcal{P}})$.

Proposition 5.6. The morphism $\zeta$ is a morphism of weight graded differential properads.

Proof. Since the definition of $\zeta$ is based on the composition $\mu$ of $\mathcal{P}$, one can see that $\zeta \circ \mu_{\mathcal{F}\left(\Sigma^{-1} \overline{\mathcal{F}}^{c}(\Sigma \mathcal{P})\right)}=\mu_{\mathcal{P}} \circ\left(\zeta \bigotimes_{c} \zeta\right)$. Hence, the morphism $\zeta$ is a morphism of properads. 
The morphism $\zeta$ is either null or given by compositions of operations which preserves the weight. Therefore, the morphism $\zeta$ is a morphism of weight graded properads.

It remains to show that $\zeta$ commutes with the differentials.

On $\overline{\mathcal{B}}^{c}(\overline{\mathcal{B}}(\mathcal{P}))=\mathcal{F}\left(\Sigma^{-1} \overline{\mathcal{F}}^{c}(\Sigma \overline{\mathcal{P}})\right)$ the differential $d$ is the sum of the derivation $d_{\theta^{\prime}}$ of the cobar construction induced by the partial coproduct of $\mathcal{F}^{c}(\Sigma \overline{\mathcal{P}})$ with the canonical differential $\delta_{\overline{\mathcal{B}}(\mathcal{P})}$. Also, the canonical differential $\delta_{\overline{\mathcal{B}}(\mathcal{P})}$ is the sum of the coderivation $d_{\theta}$ of the bar construction $\overline{\mathcal{B}}(\mathcal{P})$ induced by the partial product of $\mathcal{P}$ with the canonical differential $\delta_{\mathcal{P}}$. Let $\xi$ be an element of $\mathcal{F}\left(\Sigma^{-1} \overline{\mathcal{F}}^{c}(\Sigma \overline{\mathcal{P}})\right)$ represented by an indexed graph $g$. Three different cases are possible.

- When all the vertices of $g$ are indexed by elements of $\mathcal{F}_{(1)}^{c}(\Sigma \overline{\mathcal{P}})=\Sigma \overline{\mathcal{P}}$, the image of $\xi$ under the differential $d$ is equal to the image under the canonical differential $\delta_{\mathcal{P}}$. Since the composition $\mu$ of $\mathcal{P}$ is a morphism of differential properads, we have $\zeta \circ d(\xi)=\zeta \circ \delta_{\mathcal{P}}(\xi)=\delta_{\mathcal{P}} \circ \zeta(\xi)$.

- When at least one vertex of $g$ is indexed by an element of $\mathcal{F}_{(s)}^{c}(\Sigma \overline{\mathcal{P}})$, with $s \geq 3$, the image of $\xi$ under $\zeta$ is null. Moreover, the image of $\xi$ under the differential $d$ is a sum of graphs where at least one vertex is indexed by an element of $\mathcal{F}_{(s)}^{c}(\Sigma \overline{\mathcal{P}})$, with $s \geq 2$. Therefore, we have $d \circ \zeta(\xi)+\zeta \circ d(\xi)=0$.

- When the vertices of $g$ are indexed by elements of $\mathcal{F}_{(s)}^{c}(\Sigma \overline{\mathcal{P}})$ where $s=1,2$, with at least one of $\mathcal{F}_{(2)}^{c}(\Sigma \overline{\mathcal{P}})$, the image of $\xi$ under $\zeta$ is null. It remains to show that $\zeta \circ d(\xi)=0$. Since $\delta_{\mathcal{P}}$ preserves the natural graduation of $\mathcal{F}^{c}$, one has $\zeta \circ \delta_{\mathcal{P}}(\xi)=0$. Study the image under $d_{\theta}+d_{\theta^{\prime}}$ of an element of $\mathcal{F}_{(2)}^{c}(\Sigma \overline{\mathcal{P}})$. We denote $\xi=X \otimes \Sigma^{-1}\left(\Sigma p_{1} \otimes \Sigma p_{2}\right) \otimes Y$, where $\left(\Sigma p_{1} \otimes \Sigma p_{2}\right)$ belongs to $\mathcal{F}_{(2)}^{c}(\Sigma \overline{\mathcal{P}})$ and $X, Y$ to $\mathcal{F}\left(\Sigma^{-} 1 \overline{\mathcal{F}}^{c}(\Sigma \overline{\mathcal{P}})\right)$. Applying $d_{\theta^{\prime}}$, we get one term of the form

$$
(-1)^{|X|+1}(-1)^{\left|p_{1}\right|+1} X \otimes \Sigma^{-1} \Sigma p_{1} \otimes \Sigma^{-1} \Sigma p_{2} \otimes Y,
$$

where $\Sigma^{-1} \Sigma p_{1}$ and $\Sigma^{-1} \Sigma p_{2}$ are elements of $\Sigma^{-1} \mathcal{F}_{(1)}^{c}(\Sigma \overline{\mathcal{P}})$. Also, applying $d_{\theta}$, we get one term of the form

$$
(-1)^{|X|+1}(-1)^{\left|p_{1}\right|} X \otimes \Sigma^{-1} \Sigma \mu\left(p_{1} \otimes p_{2}\right) \otimes Y,
$$

where $\Sigma^{-1} \Sigma \mu\left(p_{1} \otimes p_{2}\right)$ belongs to $\Sigma^{-1} \mathcal{F}_{(1)}^{c}(\Sigma \overline{\mathcal{P}})$. Therefore, $\zeta \circ\left(d_{\theta}+d_{\theta^{\prime}}\right)(\xi)$ is a sum of terms of the form

$$
\left((-1)^{|X|+\left|p_{1}\right|}+(-1)^{|X|+\left|p_{1}\right|+1}\right) X \otimes \mu\left(p_{1} \otimes p_{2}\right) \otimes Y=0 .
$$

Remark. With this proof, one can understand the use of the sign -1 in the definition of the derivation $d_{\theta^{\prime}}$ of the cobar construction.

Denote $\mathcal{C}:=\overline{\mathcal{B}}(\mathcal{P})$ as the connected dg-coproperad defined by the bar construction on $\mathcal{P}$. We consider the augmented cobar construction on the right $\overline{\mathcal{B}}^{c}(\mathcal{C}) \bigotimes_{c} \mathcal{C}=$ $\overline{\mathcal{B}}^{c}(\overline{\mathcal{B}}(\mathcal{P})) \bigotimes_{c} \overline{\mathcal{B}}(\mathcal{P})$. Remark that the coproperad $\overline{\mathcal{B}}(\mathcal{P})=\mathcal{F}^{c}(\Sigma \overline{\mathcal{P}})$ is weight graded (by the global weight) and that the augmented cobar construction $\overline{\mathcal{B}}^{c}(\mathcal{C}) \bigotimes_{c} \mathcal{C}$ is an analytic quasi-cofree $\mathcal{C}$-comodule.

Lemma 5.7. The morphism $\zeta \bigotimes_{c} \overline{\mathcal{B}}(\mathcal{P}): \overline{\mathcal{B}}^{c}(\overline{\mathcal{B}}(\mathcal{P})) \bigotimes_{c} \overline{\mathcal{B}}(\mathcal{P}) \rightarrow \mathcal{P} \bigotimes_{c} \overline{\mathcal{B}}(\mathcal{P})$ between the augmented cobar construction $\overline{\mathcal{B}}^{c}(\mathcal{C}) \bigotimes_{c} \mathcal{C}$ and the augmented bar construction 
$\mathcal{P} \otimes_{c} \overline{\mathcal{B}}(\mathcal{P})$ is a morphism of $d g-\mathbb{S}$-bimodules. Moreover, it is a morphism of analytic quasi-free $\overline{\mathcal{B}}(\mathcal{P})$-comodules.

Proof. Since $\zeta$ and $i d_{\overline{\mathcal{B}}(\mathcal{P})}$ are morphisms of dg-S-bimodules, the morphism $\zeta \bigotimes_{c}$ $i d_{\overline{\mathcal{B}}(\mathcal{P})}$ preserves the canonical differentials $\delta_{\overline{\mathcal{B}}(\mathcal{C})}+\delta_{\mathcal{C}}$ and $\delta_{\mathcal{P}}+\delta_{\overline{\mathcal{B}}(\mathcal{P})}$. One can see that the morphism $d_{\theta_{r}}$ that appears in the definition of the differential of the cobar construction $\overline{\mathcal{B}}^{c}(\mathcal{C}) \otimes_{c} \mathcal{C}\left(c f\right.$. 4.2) corresponds via $\zeta \bigotimes_{c} \overline{\mathcal{B}}(\mathcal{P})$ to the morphism $d_{\theta_{l}}$ of the differential of the bar construction $\mathcal{P} \otimes_{c} \overline{\mathcal{B}}(\mathcal{P})$. (The morphism $d_{\theta_{r}}$ corresponds to extract an operation of $\mathcal{P}$ from $\mathcal{C}=\overline{\mathcal{B}}(\mathcal{P})$ from the bottom and compose it, from the top, in $\overline{\mathcal{B}}^{c}(\overline{\mathcal{B}}(\mathcal{P}))$. The morphism $d_{\theta_{l}}$ corresponds to extract an operation of $\mathcal{P}$ in $\overline{\mathcal{B}}(\mathcal{P})$ from the bottom to compose it at the top of $\mathcal{P}$.)

Since $\zeta$ and $i d_{\overline{\mathcal{B}}(\mathcal{P})}$ preserve the weight graduation coming from the one of $\mathcal{P}$, we have that $\zeta \otimes_{c} \overline{\mathcal{B}}(\mathcal{P})$ is a morphism of analytic quasi-free $\overline{\mathcal{B}}(\mathcal{P})$-comodules.

Theorem 5.8 (Bar-cobar resolution). For every connected dg-properad $\mathcal{P}$, the morphism

$$
\zeta: \overline{\mathcal{B}}^{c}(\overline{\mathcal{B}}(\mathcal{P})) \rightarrow \mathcal{P}
$$

is a quasi-isomorphism of weight graded dg-properads.

Proof. We know from Theorem 4.18 that the augmented cobar construction $\overline{\mathcal{B}}^{c}(\overline{\mathcal{B}}(\mathcal{P})) \otimes_{c} \overline{\mathcal{B}}(\mathcal{P})$ is acyclic and from Theorem 4.15 that the augmented bar construction $\mathcal{P} \otimes_{c} \overline{\mathcal{B}}(\mathcal{P})$ is acyclic too. Therefore, the morphism $\zeta \otimes_{c} \overline{\mathcal{B}}(\mathcal{P})$ is a quasiisomorphism. We apply the comparison lemma for analytic quasi-cofree comodules (Theorem 5.5) to the quasi-isomorphisms $\Psi=i d_{\overline{\mathcal{B}}(\mathcal{P})}$ and $\Phi=\zeta \otimes_{c} \overline{\mathcal{B}}(\mathcal{P})$. It gives that $\zeta=\bar{\Phi}$ is a quasi-isomorphism.

Remark. In the rest of the text, we will mainly apply this theorem to quadratic properads (which are naturally graded). The comparison lemma for quasi-free properads, proved in the next section, will allow us to simplify the bar-cobar resolution to get a quasi-free resolution, namely the Koszul resolutions ( $c f$. Section 7 ).

5.2. Comparison lemma for quasi-free properad. Here we show a comparison lemma of the same type but for quasi-free properads.

Theorem 5.9 (Comparison lemma for quasi-free properads). Let $M$ and $M^{\prime}$ be two weight graded $d g-\mathbb{S}$-bimodules such that $M^{(0)}=M^{\prime(0)}=0$. Let $\mathcal{P}$ and $\mathcal{P}^{\prime}$ be two quasi-free properads of the form $\mathcal{P}=\mathcal{F}(M)$ and $\mathcal{P}^{\prime}=\mathcal{F}\left(M^{\prime}\right)$, endowed with derivations $d_{\theta}$ and $d_{\theta^{\prime}}$ coming from decomposable morphisms of weight graded $d g$ S-bimodules $\theta: M \rightarrow \bigoplus_{s \geq 2} \mathcal{F}_{(s)}(M)$ and $\theta^{\prime}: M^{\prime} \rightarrow \bigoplus_{s \geq 2} \mathcal{F}_{(s)}\left(M^{\prime}\right)$. Given a morphism of weight graded dg-properads $\Phi: \mathcal{P} \rightarrow \mathcal{P}^{\prime}$, we consider the induced morphism $\bar{\Phi}: M=\mathcal{F}_{(1)}(M) \rightarrow M^{\prime}=\mathcal{F}_{(1)}\left(M^{\prime}\right)$.

The morphism $\Phi$ is a quasi-isomorphism if and only if $\bar{\Phi}$ is a quasi-isomorphism.

Proof. Since the morphisms $\theta$ and $\theta^{\prime}$ preserve the weight, we have that the derivations $d_{\theta}$ and $d_{\theta^{\prime}}$ preserve the weight. Therefore, the differentials $\delta_{\theta}=\delta_{M}+d_{\theta}$ and $\delta_{\theta^{\prime}}=\delta_{M^{\prime}}+d_{\theta^{\prime}}$ preserve the weight, and we can decompose the chain complexes $\mathcal{F}(M)=\bigoplus_{\rho \in \mathbb{N}} \mathcal{F}(M)^{(\rho)}$ and $\mathcal{F}\left(M^{\prime}\right)=\bigoplus_{\rho \in \mathbb{N}} \mathcal{F}\left(M^{\prime}\right)^{(\rho)}$ with the weight.

We introduce the following filtration:

$$
F_{s}(\mathcal{F}(M)):=\bigoplus_{r \geq-s} \mathcal{F}_{(r)}(M)
$$


This filtration is compatible with the preceding decomposition. To make the spectral sequences converge, we consider the sub-complexes $\mathcal{F}(M)^{(\rho)}$ and the filtration

$$
F_{s}\left(\mathcal{F}(M)^{(\rho)}\right)=\bigoplus_{r \geq-s} \mathcal{F}_{(r)}(M)^{(\rho)} .
$$

Since the weight graded dg-S-bimodules $M$ and $M^{\prime}$ are concentrated in weight $>0$, we have $\mathcal{F}_{(r)}(M)^{(\rho)}=\mathcal{F}_{(r)}\left(M^{\prime}\right)^{(\rho)}=0$ when $r>\rho$ and

$$
F_{s}\left(\mathcal{F}(M)^{(\rho)}\right)=\bigoplus_{-s \leq r \leq \rho} \mathcal{F}_{(r)}(M)^{(\rho)} .
$$

One can see that the filtration $F_{s}$ is stable under the differential $\delta_{\theta}=\delta_{M}+d_{\theta}$. (We have $\delta_{M}\left(F_{s}\right) \subset F_{s}$ and $d_{\theta}\left(F_{s}\right) \subset F_{s-1}$.) Therefore, this filtration induces a spectral sequence $E_{s, t}^{*}$. The first term of this spectral sequence is given by the formula

$$
E_{s, t}^{0}=F_{s}\left(\mathcal{F}(M)_{s+t}^{(\rho)}\right) / F_{s-1}\left(\mathcal{F}(M)_{s+t}^{(\rho)}\right)=\mathcal{F}_{-s}(M)_{s+t}^{(\rho)},
$$

and the differential $d^{0}$ is equal to the canonical differential $\delta_{M}$. From this description and the properties of $\Phi$, we get that $E_{s, t}^{0}(\Phi)=\mathcal{F}_{(-s)}(\bar{\Phi})_{s+t}$.

The filtration $F_{s}$ of $\mathcal{F}(M)^{(\rho)}$ is bounded below and exhaustive $\left(F_{-\rho-1}=0\right.$ and $\left.F_{0}=\mathcal{F}(M)^{(\rho)}\right)$. Hence, the classical convergence theorem of spectral sequences ( $c f$. W] 5.5.1) shows that the spectral sequence $E_{s, t}^{*}$ converges to the homology of $\mathcal{F}(M)^{(\rho)}$.

We can now prove the equivalence :

$(\Leftarrow)$ If $\bar{\Phi}: M \rightarrow M^{\prime}$ is a quasi-isomorphism, since the functor $\mathcal{F}$ is exact ( $c f$. Proposition 3.13), we have that

$$
\mathcal{F}_{(-s)}(\bar{\Phi})_{s+t}=E_{s, t}^{0}\left(\Phi^{(\rho)}\right): E_{s, t}^{0}\left(\mathcal{F}(M)^{(\rho)}\right) \rightarrow E_{s, t}^{0}\left(\mathcal{F}\left(M^{\prime}\right)^{(\rho)}\right)
$$

is a quasi-isomorphism. Hence, $E^{1}\left(\Phi^{(\rho)}\right)$ is an isomorphism. The convergence of the spectral sequence shows that $\Phi^{(\rho)}: \mathcal{F}(M)^{(\rho)} \rightarrow \mathcal{F}\left(M^{\prime}\right)^{(\rho)}$ is a quasi-isomorphism.

$(\Rightarrow)$ In the other way, if $\Phi$ is a quasi-isomorphism, then each $\Phi^{(\rho)}$ is a quasiisomorphism. We will show by induction on $\rho$ that $\bar{\Phi}^{(\rho)}: M^{(\rho)} \rightarrow M^{\prime(\rho)}$ is a quasi-isomorphism.

For $\rho=0$, we have $M^{(0)}=M^{\prime(0)}=0$ and the morphism $\bar{\Phi}^{(0)}$ is a quasiisomorphism.

Suppose that the result is true up to $\rho$ and show it for $\rho+1$. In this case, since $E_{s, t}^{0}\left(\mathcal{F}(M)^{(\rho+1)}\right)=\mathcal{F}_{(-s)}(M)_{s+t}^{(\rho+1)}$, one can see that $E_{s, t}^{1}\left(\Phi^{(\rho+1)}\right)$ is an isomorphism for every $t$, so long as $s<-1$.

Once again, we consider the mapping cone of the application $\Phi^{(\rho+1)}$. We define the same filtration on the mapping cone $\operatorname{cone}\left(\Phi^{(\rho+1)}\right)$ as on $\mathcal{F}(M)^{(\rho+1)}$ :

$$
F_{s}\left(\operatorname{cone}\left(\Phi^{(\rho+1)}\right)\right):=F_{s}\left(\Sigma^{-1} \mathcal{F}(M)^{(\rho+1)}\right) \oplus F_{s}\left(\mathcal{F}\left(M^{\prime}\right)^{(\rho+1)}\right) .
$$

This filtration induces a spectral sequence such that

$$
E_{s, *}^{0}\left(\operatorname{cone}\left(\Phi^{(\rho+1)}\right)\right)=\operatorname{cone}\left(E_{s, *}^{0}\left(\Phi^{\rho+1}\right)\right) .
$$

As in the proof of Theorem [5.4, we have the following long exact sequence:

$$
\begin{gathered}
\cdots \longrightarrow E_{s, t+1}^{1}\left(\mathcal{F}(M)^{(\rho+1)}\right) \longrightarrow E_{s, t+1}^{1}\left(\mathcal{F}\left(M^{\prime}\right)^{(\rho+1)}\right) \longrightarrow E_{s, t}^{1}\left(\operatorname{cone}\left(\Phi^{(\rho+1)}\right)\right) \\
\longrightarrow E_{s, t}^{1}\left(\mathcal{F}(M)^{(\rho+1)}\right) \longrightarrow E_{s, t}^{1}\left(\mathcal{F}\left(M^{\prime}\right)^{(\rho+1)}\right) \longrightarrow E_{s, t-1}^{1}\left(\operatorname{cone}\left(\Phi^{(\rho+1)}\right)\right) \longrightarrow \cdots .
\end{gathered}
$$


Since $E_{s, t}^{1}\left(\Phi^{(\rho+1)}\right)$ is an isomorphism, for $s<-1$, the long exact sequence shows that $E_{s, t}^{1}\left(\operatorname{cone}\left(\Phi^{(\rho+1)}\right)=0\right.$ for every $t$ so long as $s<-1$. The form of the filtration gives that $E_{s, t}^{1}\left(\operatorname{cone}\left(\Phi^{(\rho+1)}\right)\right)=0$ for every $t$ when $s \geq 0$. The spectral sequence $E_{s, t}^{*}\left(\operatorname{cone}\left(\Phi^{(\rho+1)}\right)\right)$ collapses at rank $E^{1}$ (only the row $s=-1$ is not null). It implies that

$$
E_{-1, t}^{\infty}\left(\operatorname{cone}\left(\Phi^{(\rho+1)}\right)\right)=E_{-1, t}^{1}\left(\operatorname{cone}\left(\Phi^{(\rho+1)}\right)\right)
$$

Applying the classical convergence theorem of spectral sequences, we get that the spectra sequence $E_{s, t}^{*}\left(\operatorname{cone}\left(\Phi^{(\rho+1)}\right)\right)$ converges to the homology of the mapping cone of $\Phi^{(\rho+1)}$ which is null since $\Phi^{(\rho+1)}$ is a quasi-isomorphism. Therefore we have $E_{-1, t}^{1}\left(\operatorname{cone}\left(\Phi^{(\rho+1)}\right)\right)=0$, for every $t$, which injected in the long exact sequence gives that

$$
E_{-1, t}^{0}\left(\Phi^{(\rho+1)}\right)=\bar{\Phi}^{(\rho+1)}: M^{(\rho+1)} \rightarrow M^{\prime(\rho+1)}
$$

is a quasi-isomorphism.

Remark. This theorem generalizes to properads a result of B. Fresse [Fr] for "connected" operads $(\mathcal{P}(0)=0$ and $\mathcal{P}(1)=k)$. This hypothesis ensures the convergence of the spectral sequence introduced by the author. To be able to generalize this result, we have introduced the graduation by the weight. Therefore, Theorem 5.9 can be applied to weight graded operads non-necessarily "connected" in the sense of $[\mathrm{Fr}]$, for instance operads with unitary operations or weight graded algebras.

Corollary 5.10. Let $\mathcal{P}=\mathcal{F}(M)$ be a quasi-free properad verifying the hypothesis of the previous theorem. The natural projection $\overline{\mathcal{B}}(\mathcal{F}(M)) \rightarrow \Sigma M$ is a quasiisomorphism.

Proof. Denote $M^{\prime}:=\Sigma^{-1} \overline{\mathcal{B}}(\mathcal{F}(M))$. The bar-cobar construction of the weight graded connected dg-properad $\mathcal{P}$ is a quasi-free properad of the form $\mathcal{B}^{c} \mathcal{B}(\mathcal{F}(M))=$ $\mathcal{F}\left(M^{\prime}\right)$, which is quasi-isomorphic to $\mathcal{P}=\mathcal{F}(M)$ by Theorem 5.8. The comparison lemma for a quasi-free properad (Theorem 5.9) concludes the proof.

\section{Simplicial BAR CONSTRUCtion}

In this section, we generalize the simplicial bar construction of associative algebras and operads to properads. We show that the simplicial bar construction of a properad is quasi-isomorphic to its differential bar construction.

6.1. Definitions. In every monoidal category $(\mathcal{A}, \square, I)$, one can associate to every monoid $M$ a simplicial chain complex on the objects $M^{\square n}$, for $n \in \mathbb{N}$. In the category of $k$-modules, one gets the classical bar construction of associative algebras. In the case of monads, one finds the triple bar construction of J. Beck $[\mathrm{B}$. We apply this construction to the monoidal category of $\mathbb{S}$-bimodules.

\subsubsection{Simplicial bar construction with coefficients.}

Definition (Simplicial bar construction with coefficients). Let $\mathcal{P}$ be a differential properad and let $(L, l)$ and $(R, r)$ be two differential $\mathcal{P}$-modules on the right and on the left. We denote

$$
\mathcal{C}_{n}(L, \mathcal{P}, R):=\Sigma^{n} L \bigotimes_{c} \underbrace{\mathcal{P} \bigotimes_{c} \cdots \bigotimes_{c} \mathcal{P}}_{n} \bigotimes_{c} R .
$$


We define the face maps $d_{i}: \mathcal{C}_{n}(L, \mathcal{P}, R) \rightarrow \mathcal{C}_{n-1}(L, \mathcal{P}, R)$ by

- the right action $l$ if $i=0$,

- the composition $\mu$ of the $i^{\text {th }}$ with the $(i+1)^{\text {th }}$ line, composed by elements of $\mathcal{P}$,

- the left action $r$ if $i=n$.

The degeneracy maps $s_{j}: \mathcal{C}_{n}(L, \mathcal{P}, R) \rightarrow \mathcal{C}_{n+1}(L, \mathcal{P}, R)$ are given by the insertion of the unit $\eta$ of the properad : $L \otimes_{c} \mathcal{P}^{\bigotimes_{c} j} \bigotimes_{c} \eta \bigotimes_{c} \mathcal{P}^{\bigotimes_{c} n-j} \bigotimes_{c} R$.

The differential $d_{\mathcal{C}}$ on $\mathcal{C}(L, \mathcal{P}, R)$ is defined by the sum of the simplicial differential with the canonical differentials

$$
d_{\mathcal{C}}:=\delta_{L}+\delta_{\mathcal{P}}+\delta_{R}+\sum_{i=0}^{n}(-1)^{i+1} d_{i}
$$

One can check that $d_{\mathcal{C}}^{2}=0$ thanks to the suspension $\Sigma^{n}$ in the definition of the $\mathcal{C}_{n}(L, \mathcal{P}, R)$.

This chain complex is called the simplicial bar construction with coefficients in $L$ and $R$.

Remark. When the $\mathbb{S}$-modules $\mathcal{P}, L$ and $R$ are concentrated in degree 0 , this construction is strictly simplicial.

Like the differential bar construction (cf. Proposition 4.9), the simplicial bar construction is an exact functor.

Proposition 6.1. The simplicial bar construction $\mathcal{C}(L, \mathcal{P}, R)$ preserves quasiisomorphisms.

Proof. We use the same proof as for Proposition 4.9 with the filtration $F_{r}:=$ $\bigoplus_{s \leq r} \mathcal{C}_{(s)}(L, \mathcal{P}, R)$, where $s$ is the number of vertices of the underlying level-graph indexed by elements of $\mathcal{P}$ (including the trivial vertices indexed by $I$ ).

We define the augmentation morphism $\varepsilon: \mathcal{C}(L, \mathcal{P}, R) \rightarrow L \bigotimes_{\mathcal{C P}} R$ by the canonical projection

$$
\mathcal{C}_{0}(L, \mathcal{P}, R)=L \bigotimes_{c} R \rightarrow L \bigotimes_{c \mathcal{P}} R .
$$

As in the case of the differential bar construction, one has a notion of reduced simplicial bar construction.

Definition (Reduced simplicial bar construction). The simplicial bar construction with trivial coefficients $\mathcal{C}(I, \mathcal{P}, I)$ is called the reduced simplicial bar construction. We denote it by $\overline{\mathcal{C}}(\mathcal{P})$.

Remark. We have previously seen that the elements of the bar construction $\mathcal{B}(L, \mathcal{P}, R)$ can be represented by graphs and that the coderivation $d_{\theta}$ corresponds to the notion of "adjacent pair composition". The elements of the simplicial bar construction can be represented by level graphs, and the faces $d_{i}$ correspond to compositions of two levels of operations.

\subsubsection{Augmented simplicial bar construction.}

Definition (Augmented simplicial bar construction). The chain complexes

$$
\mathcal{C}(I, \mathcal{P}, \mathcal{P})=\overline{\mathcal{C}}(\mathcal{P}) \otimes_{c} \mathcal{P}
$$


and $\mathcal{C}(\mathcal{P}, \mathcal{P}, I)=\mathcal{P} \otimes_{c} \overline{\mathcal{C}}(\mathcal{P})$ are called augmented simplicial bar construction on the right and on the left.

Proposition 6.2. For every properad $\mathcal{P}$ and every right $\mathcal{P}$-module $L$, the augmentation morphism

$$
\varepsilon: \mathcal{C}(L, \mathcal{P}, \mathcal{P}) \rightarrow L
$$

is a quasi-isomorphism of right simplicial $\mathcal{P}$-modules.

We have the same result on the left for every left $\mathcal{P}$-module $R$.

Proof. The proof is the same as in the case of associative algebras (cf. Séminaire Cartan [C]). We consider the following filtration:

$$
F_{r}:=\bigoplus_{s \leq r} \mathcal{C}(L, \underbrace{\mathcal{P}, \mathcal{P}}_{s})
$$

where $s$ denotes the total homological degree of elements of $\mathcal{P}$. This filtration is stable under the differential $d_{\mathcal{C}}$. It induces a spectral sequence $E_{s, t}^{*}$ such that $E_{s, t}^{0}=\mathcal{C}(L, \underbrace{\mathcal{P}, \mathcal{P}}_{s})_{s+t}$ and $d^{0}=\delta_{L}+d$. We introduce an extra degeneracy map $s_{n+1}:$

$\Sigma^{n} L \bigotimes_{c} \mathcal{P}^{\bigotimes_{c} n} \bigotimes_{c} \mathcal{P} \simeq \Sigma^{n} L \bigotimes_{c} \mathcal{P}^{\bigotimes_{c} n+1} \bigotimes_{c} I \stackrel{\Sigma^{n} L \bigotimes_{c} \mathcal{P}^{\bigotimes_{c} n+1} \bigotimes_{c} \eta}{\longrightarrow} \Sigma^{n+1} L \bigotimes_{c} \mathcal{P}^{\bigotimes_{c} n+1} \bigotimes_{c} \mathcal{P}$, which induces a contracting homotopy on $E^{0}$. Once again, we conclude with the classical convergence theorem for spectral sequences.

Corollary 6.3. The augmented simplicial bar construction on the left and on the right are acyclic.

6.1.3. Normalized bar construction. To any simplicial chain complex, one can associate its normalized chain complex, which is the quotient by the images of the degeneracy maps.

Definition (Normalized bar construction). The normalized bar construction is given by the quotient of the simplicial bar construction by the images of the degeneracies maps. We denote $\mathcal{N}_{n}(L, \mathcal{P}, R)$ as the following dg-S-module:

$\Sigma^{n} \operatorname{coker}\left(L \bigotimes_{c} \mathcal{P}^{\bigotimes_{c}(n-1)} \bigotimes_{c} R \stackrel{\sum_{i=0}^{n-1} L \bigotimes_{c} \mathcal{P}^{\bigotimes_{c} i} \bigotimes_{c} \eta \bigotimes_{c} \mathcal{P}^{\bigotimes_{c}(n-i-1)} \bigotimes_{c} R}{\longrightarrow} L \bigotimes_{c} \mathcal{P}^{\bigotimes_{c} n} \bigotimes_{c} R\right)$.

The chain complex $\mathcal{N}(I, \mathcal{P}, I)$, denoted $\overline{\mathcal{N}}(\mathcal{P})$, is called the reduced normalized bar construction.

Remark. The normalized bar construction $\mathcal{N}(L, \mathcal{P}, R)$ is isomorphic to the subcomplex of the simplicial bar construction $\mathcal{C}(L, \mathcal{P}, R)$ spanned by the level-graphs indexed by at least one element of $\overline{\mathcal{P}}$ on each level.

Proposition 6.4. The normalized bar construction $\mathcal{N}(L, \mathcal{P}, R)$ preserves quasiisomorphisms.

Proof. The proof is the same as Proposition 6.1,

We compute the homology of the reduced normalized bar construction of a quasifree properad as we did for the bar construction in Corollary 5.10. 
Proposition 6.5. Let $\mathcal{P}=\mathcal{F}(M)$ be a quasi-free properad on a weight graded dg$\mathbb{S}$-bimodule $M$ such that $M^{(0)}=0$ and where the derivation $d_{\theta}$ is decomposable, $\theta: M \rightarrow \bigoplus_{s \geq 2} \mathcal{F}_{(s)}(M)$. The natural projection $\overline{\mathcal{N}}(\mathcal{F}(M)) \rightarrow \Sigma M$ is a quasiisomorphism.

Proof. On the sub-complex $\overline{\mathcal{N}}(\mathcal{F}(M))^{(\rho)}$, composed by elements of total weight $(\rho)$, we consider the filtration $F_{r}:=\bigoplus_{s>-r} \overline{\mathcal{N}}_{(s)}(\mathcal{F}(M))^{(\rho)}$, where $s$ denotes the number of vertices indexed by elements of $M$. The differential $d_{\mathcal{N}}=d+\delta_{M}+d_{\theta}$ preserves this filtration, which induces a spectral sequence $E_{s, t}^{*}$. We have $E_{s, t}^{0}=$ $\overline{\mathcal{N}}_{(s)}(\mathcal{F}(M))_{s+t}^{(\rho)}$ and $d^{0}=d+\delta_{M}$. Therefore, $E^{0}\left(\overline{\mathcal{N}}(\mathcal{F}(M))^{(\rho)}\right)=\overline{\mathcal{N}}\left(E^{0}(\mathcal{F}(M))\right)^{(\rho)}$, and the lemma is true if it is true for a weight graded free dg-properad.

If $\mathcal{P}=\mathcal{F}(M)$ is a weight graded free dg-properad, consider the filtration $F_{r}^{\prime}:=$ $\bigoplus_{s \leq r} \overline{\mathcal{N}}(\mathcal{F}(M))^{s}$, where $s$ denotes the sum of the homological degree of the elements of $M$. One can see that the filtration is stable under the differential $d_{\mathcal{N}}=d+\delta_{M}$. We have $E_{s, t}^{0}=\overline{\mathcal{N}}_{t}(\mathcal{F}(M))_{s+t}$ and $d^{0}=d$. For every $s$, we define a contracting homotopy $h$ on $E_{s, *}^{0}$, which concludes the proof. Let $\xi$ be an element of $\overline{\mathcal{N}}_{t}(\mathcal{F}(M))$ represented by a graph $g$ with $t$ levels. Consider the operations of $\overline{\mathcal{F}}(M)$ indexing the last level and for each of them, consider the operations of $M$ that have no operation below (such that there are no outgoing edges attached to another operation). Call such operations extremal. Every outgoing edge of an extremal operation is an outgoing edge of $g$. Denote $m$ as the extremal operation of $\xi$ that has among its outgoing edges the one indexed by the smallest integer. We define the image of $\xi$ under $h$ by the level graph with $t+1$ levels indexed by $m$ on the last level and by the other operations of $\xi$ on the first levels (except the one without $m$ ).

6.2. Levelization morphism. The levelization morphism is an injective morphism between the bar construction and the normalized bar construction which induces an isomorphism in homology.

6.2.1. Definition. Let $\xi$ be an element of $\overline{\mathcal{B}}_{(n)}(\mathcal{P})=\mathcal{F}_{(n)}^{c}(\Sigma \overline{\mathcal{P}})$ represented by a graph $g_{\xi}$ with $n$ vertices ( $c f$. Figure 7 ).

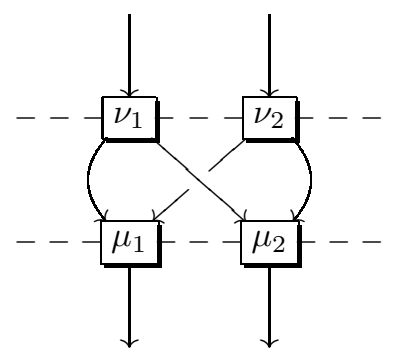

FiguRE 7. An example of $g_{\xi}$.

The element $\xi$ comes from a graph $g_{r}$ with $r$ levels under the equivalence relation $\equiv(c f$. V1] $)$. Recall that the relation $\equiv$ allows us to change the level of an operation, up to sign. With this equivalence relation, one can represent $\xi$ with at least one 
$n$-level-graph with $n$ non-trivial vertices (that's to say, with one non-trivial vertex by level $\left(c f\right.$. Figure (8)). Denote $\mathcal{G}_{n}(\xi)$ as the set of graphs with $n$ levels such that there is only one non-trivial vertex on each level, which give $g_{\xi}$ under the equivalence relation $\equiv$.

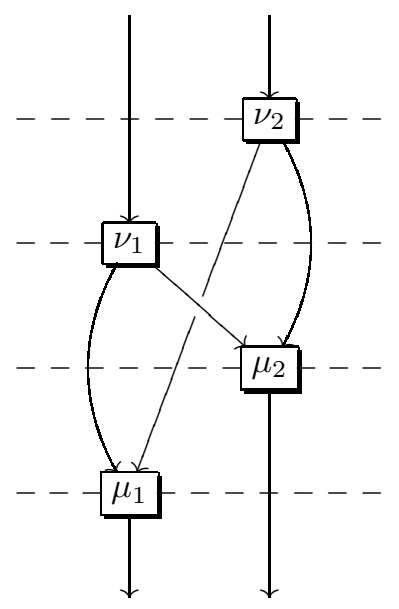

Figure 8. An example of $g \in \mathcal{G}_{4}(\xi)$.

Let $g$ be a graph of $\mathcal{G}_{n}(\xi)$. The element $\xi$ is determined by $g$ and by the sequence of operations $\Sigma p_{1} \otimes \cdots \otimes \Sigma p_{n}$ which index the vertices of $g$. To associate an element of $\overline{\mathcal{N}}(\mathcal{P})$ from $\xi$, one needs to permutate the suspensions. This makes signs appear. We denote

$$
g(\xi):=(-1)^{\sum_{i=1}^{n}(n-i)\left|p_{i}\right|} \Sigma^{n} g\left(p_{1} \otimes \cdots \otimes p_{n}\right),
$$

where $g\left(p_{1} \otimes \cdots \otimes p_{n}\right)$ corresponds to the graph $g$ whose vertices are indexed by the operations $p_{1}, \ldots, p_{n}$.

Definition (Levelization morphism). We define the levelization morphism

$$
e: \overline{\mathcal{B}}(\mathcal{P}) \rightarrow \overline{\mathcal{N}}(\mathcal{P})
$$

by the formula $e(\xi):=\sum_{g \in \mathcal{G}_{n}(\xi)} g(\xi)$, for $\xi$ in $\overline{\mathcal{B}}_{(n)}(\mathcal{P})$.

6.2.2. Homological properties. We prove that the levelization morphism is an injective quasi-isomorphism of $\mathrm{dg}$-S-bimodules.

Lemma 6.6. Let $\mathcal{P}$ be a differential augmented properad.

The levelization morphism is an injective morphism of $d g$-S-bimodules

$$
e: \overline{\mathcal{B}}(\mathcal{P}) \rightarrow \overline{\mathcal{N}}(\mathcal{P}) .
$$

Proof. Denote $d_{\mathcal{B}}$ as the differential of $\overline{\mathcal{B}}(\mathcal{P})$. It is the sum of 2 terms : $d_{\mathcal{B}}=\delta_{\mathcal{P}}+d_{\theta}$.

Call $d_{\mathcal{N}}$ the differential on $\overline{\mathcal{N}}(\mathcal{P})$. It is the sum of 2 terms. The image of an element $\tau^{\prime}=\Sigma^{n} \tau$ of $\overline{\mathcal{N}}(\mathcal{P})$ under $d_{\mathcal{N}}$ is

$$
d_{\mathcal{N}}\left(\tau^{\prime}\right)=(-1)^{n} \sum^{n} \delta_{\mathcal{P}}(\tau)+\sum_{i=1}^{n-1}(-1)^{i+1} \sum^{n-1} d_{i}(\tau) .
$$


Show that $d_{\mathcal{N}} \circ e(\xi)=e \circ d_{\mathcal{B}}(\xi)$.

- The commutativity of the canonical differentials $\delta_{\mathcal{P}} \circ e(\xi)=e \circ \delta_{\mathcal{P}}(\xi)$ comes from the good choice of signs and permutation of suspensions.

- The coderivation $d_{\theta}$ composes pairs of adjacent operations of $\Sigma \overline{\mathcal{P}}$ of the graph representing $\xi$. Also, $d_{i} \circ e$ corresponds to the composition of 2 levels of operations of $\overline{\mathcal{P}}$. Two cases must be distinguished. Denote $\xi=$ $X \otimes \Sigma p \otimes \Sigma q \otimes Y$.

(1) If the operations $\Sigma p$ and $\Sigma q$ are linked by at least an edge, then the part of $\sum_{i=1}^{n-1}(-1)^{i+1} d_{i} \circ e(\xi)$ involving the composition of $p$ with $q$ is of the form

$$
\sum \varepsilon(-1)^{j+2} \Sigma^{n-1} X^{\prime} \otimes \mu(p \otimes q) \otimes Y^{\prime},
$$

where $X^{\prime}=p_{1} \otimes \cdots \otimes p_{j}, Y^{\prime}=q_{1} \otimes \cdots \otimes q_{n-j-2} \otimes \rho_{1} \otimes \cdots \otimes \rho_{r}$ and

$$
\varepsilon=(-1)^{\sum_{i=1}^{j}(n-i)\left|p_{i}\right|+(n-j-1)|p|+(n-j-2)|q|+\sum_{k=1}^{n-j-2}(n-j-k-2)\left|q_{k}\right|} .
$$

Also, the part of $d_{\theta}(\xi)$ involving the composition of $\Sigma p$ with $\Sigma q$ is of the form

$$
(-1)^{|X|+|p|} X \otimes \Sigma \mu(p \otimes q) \otimes Y .
$$

The image of such an element under $e$ is

$$
\begin{aligned}
& \sum(-1)^{|X|+|p|} \varepsilon(-1)^{\left|X^{\prime}\right|+|p|} \Sigma^{n-1} X^{\prime} \otimes \mu(p \otimes q) \otimes Y \\
& =\sum \varepsilon(-1)^{j} \Sigma^{n-1} X^{\prime} \otimes \mu(p \otimes q) \otimes Y^{\prime} .
\end{aligned}
$$

(2) If the operations $\Sigma p$ and $\Sigma q$ are not linked by an edge, then $d_{\theta}$ does not compose $\Sigma p$ with $\Sigma q$. Also, the part of $\sum_{i=0}^{n}(-1)^{i+1} d_{i} \circ e(\xi)$ that comes from the composition of the levels where $p$ and $q$ lie is the sum of the 2 following terms :

$$
\begin{aligned}
& \sum(-1)^{j} \Sigma^{n} d_{j+1}\left(\varepsilon X^{\prime} \otimes p \otimes q \otimes Y^{\prime}+\varepsilon(-1)^{(|p|+1)(|q|+1)+|p|+|q|} X^{\prime} \otimes q \otimes p \otimes Y^{\prime}\right) \\
= & \sum(-1)^{j} \Sigma^{n}\left(\varepsilon X^{\prime} \otimes p \otimes q \otimes Y^{\prime}+\varepsilon(-1)^{(|p|+1)(|q|+1)+|p|+|q|+|p||q|} X^{\prime} \otimes p \otimes q \otimes Y^{\prime}\right) \\
= & 0 .
\end{aligned}
$$

We conclude by remarking that the image of $\xi$ under $\sum_{i=1}^{n-1}(-1)^{i+1} d_{i} \circ e-$ $e \circ d_{\theta}$ is a sum of terms of the form (1) or (2).

Now show the injectivity of $e$. Let $\xi$ be an element of $\overline{\mathcal{B}}_{(n)}(\mathcal{P})=\mathcal{F}_{(n)}^{c}(\Sigma \overline{\mathcal{P}})$. By definition of the cofree (connected) coproperad, one knows that $\xi=\xi_{1}+\cdots+\xi_{r}$ where each $\xi_{i}$ is a finite sum of elements of $\mathcal{F}_{(n)}^{c}(\Sigma \overline{\mathcal{P}})$ coming from indexing the vertices of the same graph $g_{\xi_{i}}$. We introduce a morphism $\pi: \overline{\mathcal{N}}_{n}(\mathcal{P}) \rightarrow \overline{\mathcal{B}}_{(n)}(\mathcal{P})$. To any element $\tau$ of $\overline{\mathcal{N}}_{n}(\mathcal{P})$ represented by a graph with $n$ levels, we associate its image $\pi(\tau)$ under the equivalent relation $\equiv$. Therefore, we have $\pi \circ e(\xi)=n_{1} \xi_{1}+\cdots n_{r} \xi_{r}$ where the $n_{i}$ are integers $>0$. The equation $e(\xi)=0$ gives $n_{1} \xi_{1}+\cdots+n_{r} \xi_{r}=0$. By identification of the underlying graphs, we have $\xi_{i}=0$, for all $i$, hence $\xi=0$.

The following theorem answers a question raised by M. Markl and A.A. Voronov in $[\mathrm{MV}]$ and generalizes a result of B. Fresse for operads in $\mathrm{Fr}$. 
Theorem 6.7 1 Let $\mathcal{P}$ be a weight graded augmented dg-properad.

The levelization morphism

$$
e: \overline{\mathcal{B}}(\mathcal{P}) \rightarrow \overline{\mathcal{N}}(\mathcal{P})
$$

is a quasi-isomorphism.

Proof. Since the reduced bar construction (cf. Proposition 4.9) and the reduced simplicial bar construction ( $c f$. Proposition 6.1) preserve quasi-isomorphisms, it is enough to prove this theorem for a resolution of $\mathcal{P}$. The properad $\mathcal{P}$ is weight graded and augmented. Therefore, Theorem 5.8 shows that the bar-cobar construction $\overline{\mathcal{B}}^{c}(\overline{\mathcal{B}}(\mathcal{P}))$ of $\mathcal{P}$ is a quasi-free resolution of $\mathcal{P}$. Denote $M=\Sigma^{-1} \overline{\mathcal{B}}(\mathcal{P})$ and $\mathcal{P}^{\prime}=\overline{\mathcal{B}}^{c}(\overline{\mathcal{B}}(\mathcal{P}))=\mathcal{F}(M)$. Corollary 5.10 shows that $\overline{\mathcal{B}}\left(\mathcal{P}^{\prime}\right)=\overline{\mathcal{B}}(\mathcal{F}(M))$ is quasi-isomorphic to $\Sigma M$, and Proposition 6.5 shows that $\overline{\mathcal{N}}\left(\mathcal{P}^{\prime}\right)=\overline{\mathcal{N}}(\mathcal{F}(M))$ is quasi-isomorphic to $\Sigma M$. We have the following commutative diagram of quasiisomorphism:

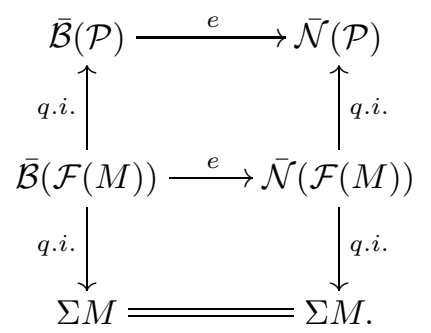

Remark. Using the same theorem for operads ( $c f$. $[$ Fr $)$, the author has proved in V3] a correspondence between operads and posets (partially ordered sets). To a set operad $\mathcal{P}$, one can associate a family of posets $\Pi_{\mathcal{P}}$ of partition type. The main theorem of [V3] asserts that the operad is Koszul over $\mathbb{Z}$ if and only if each poset of $\Pi_{\mathcal{P}}$ is Cohen-Macaulay. It seems that the same kind of work can be done in the case of a properad with the previous theorem.

\section{Koszul Duality}

We deduce the Koszul duality theory of properads from the comparison lemmas of the previous section.

7.1. Koszul dual. To a weight graded properad $\mathcal{P}$, we define its Koszul dual as a sub-coproperad of its reduced bar construction. Dually, to a weight graded coproperad $\mathcal{C}$, we define its Koszul dual as a quotient properad of its reduced cobar construction.

7.1.1. Koszul dual of a properad. Let $\mathcal{P}$ be a weight graded augmented dgproperad. Recall that the reduced bar construction $\overline{\mathcal{B}}(\mathcal{P})=\mathcal{F}^{c}(\Sigma \overline{\mathcal{P}})$ of $\mathcal{P}$ is bigraded by the number $(s)$ of non-trivial indexed vertices and by the total weight $(\rho)$

$$
\overline{\mathcal{B}}_{(s)}(\mathcal{P})=\bigoplus_{\rho \in \mathbb{N}} \overline{\mathcal{B}}_{(s)}(\mathcal{P})^{(\rho)}
$$

\footnotetext{
${ }^{1}$ The proof of this theorem was given to the author by Benoit Fresse.
} 
Since the coderivation $d_{\theta}$, induced by the partial product, composes pairs of adjacent vertices, we have

$$
d_{\theta}\left(\overline{\mathcal{B}}_{(s)}(\mathcal{P})^{(\rho)}\right) \subset\left(\overline{\mathcal{B}}_{(s-1)}(\mathcal{P})^{(\rho)}\right) .
$$

When $\mathcal{P}$ is connected $\left(\mathcal{P}^{(0)}=I\right)$, the bar construction of $\mathcal{P}$ has the following form.

Lemma 7.1. Let $\mathcal{P}$ be a weight graded connected dg-properad. We have the equalities

$$
\left\{\begin{array}{l}
\overline{\mathcal{B}}_{(\rho)}(\mathcal{P})^{(\rho)}=\mathcal{F}_{(\rho)}^{c}\left(\Sigma \overline{\mathcal{P}}^{(1)}\right), \\
\overline{\mathcal{B}}_{(s)}(\mathcal{P})^{(\rho)}=0 \quad \text { if } \quad s>\rho .
\end{array}\right.
$$

Remark. The same result holds for the reduced cobar construction of a weight graded connected dg-coproperad.

Definition (Koszul dual of a properad). Let $\mathcal{P}$ be a weight graded connected dg-properad. We define the Koszul dual of $\mathcal{P}$ by the weight graded dg-S-bimodule

$$
\mathcal{P}_{(\rho)}^{\mathrm{i}}:=H_{(\rho)}\left(\overline{\mathcal{B}}_{*}(\mathcal{P})^{(\rho)}, d_{\theta}\right) .
$$

The preceding lemma gives the form of the chain complex $\left(\overline{\mathcal{B}}_{*}(\mathcal{P})^{(\rho)}, d_{\theta}\right)$ :

$$
\ldots \stackrel{d_{\theta}}{\longrightarrow} 0 \stackrel{d_{\theta}}{\longrightarrow} \overline{\mathcal{B}}_{(\rho)}(\mathcal{P})^{(\rho)} \stackrel{d_{\theta}}{\longrightarrow} \overline{\mathcal{B}}_{(\rho-1)}(\mathcal{P})^{(\rho)} \stackrel{d_{\theta}}{\longrightarrow} \cdots .
$$

Hence, we have the formula

$$
\mathcal{P}_{(\rho)}^{\mathrm{i}}=\operatorname{ker}\left(d_{\theta}: \overline{\mathcal{B}}_{(\rho)}(\mathcal{P})^{(\rho)} \rightarrow \overline{\mathcal{B}}_{(\rho-1)}(\mathcal{P})^{(\rho)}\right) .
$$

This formula shows that $\mathcal{P}_{(\rho)}^{\mathrm{i}}$ is a weight graded dg-S-bimodule, where the differential is induced by the one of $\mathcal{P}: \delta_{\mathcal{P}}$.

If the properad is concentrated in homological degree 0 , we have

$$
\left(\overline{\mathcal{B}}_{(s)}(\mathcal{P})^{(\rho)}\right)_{d}= \begin{cases}\overline{\mathcal{B}}_{(s)}(\mathcal{P})^{(\rho)} & \text { if } d=s, \\ 0 & \text { otherwise. }\end{cases}
$$

The dual coproperad is not necessarily concentrated in degree 0 . It verifies

$$
\left(\mathcal{P}_{(\rho)}^{\mathrm{i}}\right)_{d}= \begin{cases}\mathcal{P}_{(\rho)}^{\mathrm{i}} & \text { if } d=\rho, \\ 0 & \text { otherwise }\end{cases}
$$

7.1.2. Koszul dual of a coproperad. In the same way, we define the Koszul dual of a coproperad.

Definition (Koszul dual of a coproperad). Let $\mathcal{C}$ be a weight graded connected dgcoproperad. We define the Koszul dual of $\mathcal{C}$ by the weight graded dg-S-bimodule

$$
\mathcal{C}_{(\rho)}^{\mathrm{i}}:=H_{(\rho)}\left(\overline{\mathcal{B}}_{*}^{c}(\mathcal{C})^{(\rho)}, d_{\theta^{\prime}}\right) .
$$

The cobar construction endowed with the derivation $d_{\theta^{\prime}}$ is a chain complex of the following form:

$$
\cdots \stackrel{d_{\theta^{\prime}}}{\longrightarrow} \overline{\mathcal{B}}_{(\rho-1)}(\mathcal{C})^{(\rho)} \stackrel{d_{\theta^{\prime}}}{\longrightarrow} \overline{\mathcal{B}}_{(\rho)}(\mathcal{C})^{(\rho)} \stackrel{d_{\theta^{\prime}}}{\longrightarrow} 0 .
$$

Therefore, the Koszul dual of a coproperad is isomorphic to

$$
\mathcal{C}_{(\rho)}^{\mathrm{i}}=\operatorname{coker}\left(d_{\theta^{\prime}}: \overline{\mathcal{B}}_{(\rho-1)}^{c}(\mathcal{C})^{(\rho)} \rightarrow \overline{\mathcal{B}}_{(\rho)}^{c}(\mathcal{C})^{(\rho)}\right) .
$$


The module $\mathcal{C}_{(\rho)}^{\mathrm{i}}$ is a weight graded $\mathrm{dg}$ - $\mathbb{S}$-bimodule, where the differential is induced by the one of $\mathcal{C}: \delta_{\mathcal{C}}$.

Proposition 7.2. Let $\mathcal{P}$ be weight graded connected dg-properad. The Koszul dual of $\mathcal{P}$, denoted $\mathcal{P}^{i}$, is a weight graded sub-dg-coproperad of $\mathcal{F}^{c}\left(\Sigma \mathcal{P}^{(1)}\right)$.

Let $\mathcal{C}$ be a weight graded connected dg-coproperad. The Koszul dual of $\mathcal{C}$, denoted $\mathcal{C}^{i}$, is a weight graded dg-properad quotient of $\mathcal{F}\left(\Sigma^{-1} \mathcal{C}^{(1)}\right)$.

Proof. Since $\mathcal{P}_{(\rho)}^{\mathrm{i}}=\operatorname{ker}\left(d_{\theta}: \overline{\mathcal{B}}_{(\rho)}(\mathcal{P})^{(\rho)} \rightarrow \overline{\mathcal{B}}_{(\rho-1)}(\mathcal{P})^{(\rho)}\right)$, we have that $\mathcal{P}_{(\rho)}^{\mathrm{i}}$ is a weight graded sub-dg-S-bimodule of $\mathcal{F}_{(\rho)}^{c}\left(\Sigma \mathcal{P}^{(1)}\right)$. It remains to show that $\mathcal{P}$ i is stable under the coproduct $\Delta$ of $\mathcal{F}^{c}\left(\Sigma \mathcal{P}^{(1)}\right)$. Let $\xi$ be an element of $\mathcal{P}_{(\rho)}^{\mathrm{i}}$, that's to say, $\xi \in \mathcal{F}_{(\rho)}^{c}\left(\Sigma \mathcal{P}^{(1)}\right)$ and $d_{\theta}(\xi)=0$. Denote

$$
\Delta(\xi)=\sum_{\Xi}\left(\xi_{1}^{1}, \ldots, \xi_{a_{1}}^{1}\right) \sigma\left(\xi_{1}^{2}, \ldots, \xi_{a_{2}}^{2}\right),
$$

where the sum is on a family $\Xi$ of 2-level graphs, with $\xi_{i}^{j} \in \mathcal{F}_{\left(s_{i j}\right)}^{c}\left(\Sigma \mathcal{P}^{(1)}\right)$. The fact that $d_{\theta}$ is a coderivation means that $d_{\theta} \circ \Delta(\xi)=\Delta \circ d_{\theta}(\xi)$. Therefore, we have

$$
\begin{aligned}
d_{\theta} \circ \Delta(\xi) & =\sum_{\Xi}\left(\sum_{k=1}^{a_{1}} \pm\left(\xi_{1}^{1}, \ldots, d_{\theta}\left(\xi_{k}^{1}\right), \ldots, \xi_{a_{1}}^{1}\right) \sigma\left(\xi_{1}^{2}, \ldots, \xi_{a_{2}}^{2}\right)\right. \\
& \left.+\sum_{k=1}^{a_{2}} \pm\left(\xi_{1}^{1}, \ldots, \xi_{a_{1}}^{1}\right) \sigma\left(\xi_{1}^{2}, \ldots, d_{\theta}\left(\xi_{k}^{2}\right) \ldots, \xi_{a_{2}}^{2}\right)\right)=0,
\end{aligned}
$$

where the $d_{\theta}\left(\xi_{i}^{j}\right)$ belong to $\mathcal{F}_{\left(s_{i j}-1\right)}^{c}\left(\Sigma\left(\mathcal{P}^{(1)} \oplus \mathcal{P}^{(2)}\right)\right)$ with only one vertex indexed by $\Sigma \mathcal{P}^{(2)}$. By identification, we have $d_{\theta}\left(\xi_{i}^{j}\right)=0$ for every $i, j$.

In the same way, one can see that the Koszul dual of $\mathcal{C}$ is a quotient of $\mathcal{F}\left(\Sigma^{-1} \mathcal{C}^{(1)}\right)$. It remains to show that the product $\mu$ on $\mathcal{F}\left(\Sigma^{-1} \mathcal{C}^{(1)}\right)$ defines a product on the quotient. We consider the product

$$
\mu\left(\left(c_{1}^{1}, \ldots, d_{\theta^{\prime}}(c), \ldots, c_{a_{1}}^{1}\right) \sigma\left(c_{1}^{2}, \ldots, c_{a_{2}}^{2}\right)\right),
$$

where the $c_{i}^{j}$ belong to $\mathcal{F}_{\left(s_{i j}\right)}\left(\Sigma^{-1} \mathcal{C}^{(1)}\right)$ and the $c$ to $\mathcal{F}_{(s)}\left(\Sigma^{-1}\left(\mathcal{C}^{(1)} \oplus \mathcal{C}^{(2)}\right)\right)$ with one vertex indexed by $\mathcal{C}^{(2)}$. Since the $c_{i}^{j}$ are elements of $\mathcal{F}_{\left(s_{i j}\right)}\left(\Sigma^{-1} \mathcal{C}^{(1)}\right)$, we have $d_{\theta^{\prime}}\left(c_{i}^{j}\right)=0$. The fact that $d_{\theta^{\prime}}$ is a derivation gives

$$
\begin{aligned}
& \mu\left(\left(c_{1}^{1}, \ldots, d_{\theta^{\prime}}(c), \ldots, c_{a_{1}}^{1}\right) \sigma\left(c_{1}^{2}, \ldots, c_{a_{2}}^{2}\right)\right) \\
= & d_{\theta^{\prime}}\left(\mu\left(\left(c_{1}^{1}, \ldots, c, \ldots, c_{a_{1}}^{1}\right) \sigma\left(c_{1}^{2}, \ldots, c_{a_{2}}^{2}\right)\right)\right) .
\end{aligned}
$$

Therefore the product $\mu\left(\left(c_{1}^{1}, \ldots, d_{\theta^{\prime}}(c), \ldots, c_{a_{1}}^{1}\right) \sigma\left(c_{1}^{2}, \ldots, c_{a_{2}}^{2}\right)\right)$ is null on the cokernel of $d_{\theta^{\prime}}$.

7.1.3. Quadratic properad. We show here that the dual construction is a quadratic construction.

We recall from 2.9 that a quadratical properad is a properad of the form $\mathcal{F}(V) /$ $(R)$ where $R$ belongs to $\mathcal{F}_{(2)}(V)$. If we consider the weight given by the number of indexed vertices, the ideal $(R)$ is homogenous and the quadratic properad is weight graded. 
Every quadratic properad is completely determined by its graduation

$$
\mathcal{P}=\bigoplus_{\rho \in \mathbb{N}} \mathcal{P}_{(\rho)},
$$

the dg-S-bimodule $\mathcal{P}_{(1)}$ and the dg-S-bimodule $\mathcal{P}_{(2)}$. One has $V=\mathcal{P}_{(1)}$ and $R=$ $\operatorname{ker}\left(\mathcal{F}_{(2)}\left(\mathcal{P}_{(1)}\right) \stackrel{\mu}{\rightarrow} \mathcal{P}_{(2)}\right)$.

Lemma 7.3. Let $\mathcal{C}$ be a weight graded connected dg-coproperad. The Koszul dual $\mathcal{C}^{i}$ is a quadratic properad generated by

$$
\mathcal{C}_{(1)}^{i}=\Sigma^{-1} \mathcal{C}^{(1)} \quad \text { and } \quad \mathcal{C}_{(2)}^{i}=\operatorname{coker}\left(\theta^{\prime}: \Sigma^{-1} \mathcal{C}^{(2)} \rightarrow \mathcal{F}_{(2)}\left(\Sigma^{-1} \mathcal{C}^{(1)}\right)\right) .
$$

Proof. Denote $R=\operatorname{ker}\left(\mathcal{F}_{(2)}\left(\mathcal{C}_{(1)}^{\mathrm{i}}\right) \rightarrow \mathcal{C}_{(2)}^{\mathrm{i}}\right)=\operatorname{Im}\left(\theta_{\Sigma^{-1} \mathcal{C}^{(2)}}^{\prime}\right)$. By definition, $\mathcal{C}_{(\rho)}^{\mathrm{i}}$ is the quotient of $\mathcal{F}_{(\rho)}\left(\Sigma^{-1} \mathcal{C}^{(1)}\right)$ by the image of $d_{\theta^{\prime}}$ on $\overline{\mathcal{B}}_{(\rho-1)}^{c}\left(\mathcal{C}^{(\rho)}\right)$. This image corresponds to graphs with $\rho$ vertices such that at least one pair of adjacent vertices is the image of an element of $\Sigma^{-1} \mathcal{C}^{(2)}$ under $\theta^{\prime}$. This is isomorphic to the part of weight $\rho$ of the ideal generated by $R$. Therefore, we have $\mathcal{C}^{\mathrm{i}}=\mathcal{F}\left(\Sigma^{-1} \mathcal{C}^{(1)}\right) /(R)$.

Remark. Dually, one can define the notion of quadratic coproperad and prove that the Koszul dual of a properad is a quadratic coproperad.

7.2. Koszul properads and Koszul resolutions. Here we give the notions of Koszul properad and coproperad. When a properad $\mathcal{P}$ is a Koszul properad, it is quadratic, its dual is Koszul and its bidual is isomorphic to $\mathcal{P}$. We prove that a properad $\mathcal{P}$ is Koszul if and only if the reduced cobar construction of $\mathcal{P}^{i}$ is a resolution of $\mathcal{P}$.

\subsubsection{Definitions.}

Definition (Koszul properad). Let $\mathcal{P}$ be a weight graded connected dg-proper ad. The properad $\mathcal{P}$ is a Koszul properad if the natural inclusion $\mathcal{P} i \hookrightarrow \overline{\mathcal{B}}(\mathcal{P})$ is a quasi-isomorphism.

In the same way, we have the definition of a Koszul coproperad.

Definition (Koszul coproperad). Let $\mathcal{C}$ be a weight graded connected dg-coproperad. We call $\mathcal{C}$ a Koszul coproperad if the natural projection $\overline{\mathcal{B}}^{c}(\mathcal{C}) \rightarrow \mathcal{C}^{i}$ is a quasiisomorphism.

Proposition 7.4. If $\mathcal{P}$ is a weight graded Koszul properad, then its Koszul dual $\mathcal{P} i$ is a Koszul coproperad and $\mathcal{P}^{i}=\mathcal{P}$.

Proof. The properad $\mathcal{P}$ is concentrated in (homological) degree $0\left(\delta_{\mathcal{P}}=0\right.$ and $\left.\mathcal{P}_{0}=\mathcal{P}\right)$. In this case, $\mathcal{P}_{(\rho)}^{\mathrm{i}}$ is a homogenous $\mathbb{S}$-bimodule of degree $\rho$ and the elements of degree 0 of $\overline{\mathcal{B}}^{c}\left(\mathcal{P}^{\mathrm{i}}\right)^{(\rho)}$ correspond to the elements of $\overline{\mathcal{B}}_{(\rho)}^{c}\left(\mathcal{P}^{\mathrm{i}}\right)^{(\rho)}$. This shows that

$$
H_{0}\left(\overline{\mathcal{B}}_{(\rho)}^{c}\left(\mathcal{P}^{\mathrm{i}}\right)^{(\rho)}\right)=H_{\rho}\left(\overline{\mathcal{B}}_{*}^{c}\left(\mathcal{P}^{\mathrm{i}}\right)^{(\rho)}, d_{\theta^{\prime}}\right)=\mathcal{P}^{\mathrm{i}(\rho)} .
$$

Since the properad $\mathcal{P}$ is Koszul, the inclusion $\mathcal{P} i \hookrightarrow \overline{\mathcal{B}}(\mathcal{P})$ is a quasi-isomorphism. Using the comparison lemma for quasi-free properads (Theorem [5.9), we show that the induced morphism $\overline{\mathcal{B}}^{c}\left(\mathcal{P}^{i}\right) \rightarrow \overline{\mathcal{B}}^{c}(\overline{\mathcal{B}}(\mathcal{P}))$ is a quasi-isomorphism. Since the barcobar construction is a resolution of $\mathcal{P}$ (Theorem 5.8), the cobar construction $\overline{\mathcal{B}}^{c}\left(\mathcal{P}^{\mathrm{i}}\right)$ 
is quasi-isomorphic to $\mathcal{P}$. The $\mathbb{S}$-bimodule $\mathcal{P}$ is homogenous of degree 0 , therefore we have

$$
H_{*}\left(\overline{\mathcal{B}}^{c}\left(\mathcal{P}^{\mathrm{i}}\right)^{(\rho)}\right)= \begin{cases}\mathcal{P}^{(\rho)} & \text { if } *=0 \\ 0 & \text { otherwise. }\end{cases}
$$

Finally, we get that $\mathcal{P}^{i}$ is a Koszul coproperad and that $\mathcal{P}^{i}=\mathcal{P}$.

Corollary 7.5. Let $\mathcal{P}$ be a weight graded connected properad. If $\mathcal{P}$ is a Koszul properad, then $\mathcal{P}$ is quadratic.

Proof. If $\mathcal{P}$ is a Koszul properad, by the preceding proposition, we know that $\mathcal{P}=\mathcal{P}^{i^{i}}$. Also, Lemma 7.3 shows that $\mathcal{P}^{i}$ is quadratic.

7.2.2. Koszul resolution and minimal model. We have seen in the proof of the previous proposition that, when $\mathcal{P}$ is a Koszul properad, the quasi-isomorphism $\mathcal{P} \mathrm{i} \hookrightarrow \overline{\mathcal{B}}(\mathcal{P})$ induces a quasi-isomorphism $\overline{\mathcal{B}}^{c}(\mathcal{P} \mathrm{i}) \rightarrow \overline{\mathcal{B}}^{c}(\overline{\mathcal{B}}(\mathcal{P}))$. When we compose this quasi-isomorphism with the bar-cobar resolution $\zeta: \overline{\mathcal{B}}^{c}(\overline{\mathcal{B}}(\mathcal{P})) \rightarrow \mathcal{P}(c f$. Theorem 5.8 ), we get a quasi-isomorphism of weight graded dg-properads:

$$
\overline{\mathcal{B}}^{c}\left(\mathcal{P}^{\mathrm{i}}\right) \rightarrow \mathcal{P} .
$$

Definition (Koszul resolution). When $\mathcal{P}$ is a Koszul properad, the resolution $\overline{\mathcal{B}}^{c}\left(\mathcal{P}^{i}\right) \rightarrow \mathcal{P}$ is called a Koszul resolution.

Moreover, we have the following equivalence.

Theorem 7.6 (Koszul resolution). Let $\mathcal{P}$ be a weight graded connected dg-properad. The properad $\mathcal{P}$ is a Koszul properad if and only if the morphism of weight graded $d g$-properads $\overline{\mathcal{B}}^{c}\left(\mathcal{P}^{i}\right) \rightarrow \mathcal{P}$ is a quasi-isomorphism.

Proof. On the other hand, if the morphism $\overline{\mathcal{B}}^{c}\left(\mathcal{P}^{i}\right) \rightarrow \mathcal{P}$ is a quasi-isomorphism, then $\overline{\mathcal{B}}^{c}(\mathcal{P} \mathrm{i}) \rightarrow \overline{\mathcal{B}}^{c}(\overline{\mathcal{B}}(\mathcal{P}))$ is also a quasi-isomorphism. Since we work with weight graded quasi-free properads, we can apply the comparison lemma for quasi-free properads (Theorem [5.9) which gives that the inclusion $\mathcal{P}^{i} \hookrightarrow \overline{\mathcal{B}}(\mathcal{P})$ is a quasiisomorphism.

When $\mathcal{P}$ is a Koszul properad concentrated in degree 0 , we have a resolution $\overline{\mathcal{B}}^{c}\left(\mathcal{P}^{\mathrm{i}}\right)=\mathcal{F}^{c}\left(\Sigma^{-1} \overline{\mathcal{P}}^{\mathrm{i}}\right) \rightarrow \mathcal{P}$ built on a quasi-free properad and such that the differential is quadratic $d_{\theta^{\prime}}\left(\Sigma^{-1} \overline{\mathcal{P}}^{\mathrm{i}}\right) \subset \mathcal{F}_{(2)}\left(\Sigma^{-1} \overline{\mathcal{P}}^{\mathrm{i}}\right)$. By analogy with associative algebras and operads, we call this resolution the minimal model of $\mathcal{P}$.

7.3. Koszul complex. It is as difficult to show that a properad $\mathcal{P}$ is a Koszul properad from the definition ( $\mathcal{P} i \rightarrow \overline{\mathcal{B}}(\mathcal{P})$ quasi-isomorphism) as to show that the cobar construction of $\mathcal{P}^{i}$ is a resolution of $\mathcal{P}\left(\overline{\mathcal{B}}^{c}(\mathcal{P} i) \rightarrow \mathcal{P}\right.$ quasi-isomorphism). We introduce a smaller chain complex whose acyclicity is a criterion which proves that $\mathcal{P}$ is a Koszul properad.

7.3.1. Koszul complex with coefficients. Following the same method as in the definition of the bar construction with coefficients ( $c f$. 4.2.2), here we introduce the notion of a Koszul complex with coefficients.

Definition (Koszul complex with coefficients). Let $\mathcal{P}$ be a weight graded connected dg-properad and let $L$ and $R$ be two modules (on the right and on the left) on $\mathcal{P}$. We call a Koszul complex with coefficients in the modules $L$ and $R$, the chain complex 
defined on the $\mathbb{S}$-bimodule $L \bigotimes_{c} \mathcal{P}$ i $\bigotimes_{c} R$ by the differential $d$, the sum of the three following terms :

(1) the canonical differentials $\delta_{\mathcal{P}}, \delta_{R}$ and $\delta_{L}$ induced by the ones of $\mathcal{P}, R$ and $L$.

(2) The homogenous morphism $d_{\theta_{L}}$ of degree -1 that comes from the structure of $\mathcal{P}$-comodule on the left on $\mathcal{P i}^{\mathrm{i}}$ :

$$
\theta_{l}: \mathcal{P}^{\mathrm{i}} \stackrel{\Delta}{\longrightarrow} \mathcal{P}^{\mathrm{i}} \bigotimes_{c} \mathcal{P}^{\mathrm{i}} \rightarrow(I \oplus \underbrace{\overline{\mathcal{P}}^{\mathrm{i}}}_{1}) \bigotimes_{c} \mathcal{P}^{\mathrm{i}} \rightarrow(I \oplus \underbrace{\mathcal{P}^{(1)}}_{1}) \bigotimes_{c} \mathcal{P}^{\mathrm{i}} .
$$

(3) The homogenous morphism $d_{\theta_{R}}$ of degree -1 that comes from the structure of $\mathcal{P}$-comodule on the right on $\mathcal{P}^{i}$ :

$$
\theta_{r}: \mathcal{P}^{\mathrm{i}} \stackrel{\Delta}{\longrightarrow} \mathcal{P}^{\mathrm{i}} \otimes_{c} \mathcal{P}^{\mathrm{i}} \rightarrow \mathcal{P}^{\mathrm{i}} \otimes_{c}(I \oplus \underbrace{\overline{\mathcal{P}^{\mathrm{i}}}}_{1}) \rightarrow \mathcal{P}^{\mathrm{i}} \otimes_{c}(I \oplus \underbrace{\mathcal{P}^{(1)}}_{1}) .
$$

We denote it $\mathcal{K}(L, \mathcal{P}, R)$.

Since $\mathcal{P}$ i can be injected into the bar construction $\overline{\mathcal{B}}(\mathcal{P})$, the Koszul complex with coefficients is a sub-S-bimodule of the bar construction with coefficients.

Proposition 7.7. Let $\mathcal{P}$ be a weight graded connected dg-properad and let $L$ and $R$ be two modules (on the right and on the left) on $\mathcal{P}$. The dg-S-bimodule $\mathcal{K}(L, \mathcal{P}, R)=$ $L \bigotimes_{c} \mathcal{P} i \bigotimes_{c} R$ is a sub-complex of the bar construction with coefficients $\mathcal{B}(L, \mathcal{P}, R)=$ $L \otimes_{c} \overline{\mathcal{B}}(\mathcal{P})_{c} \otimes R$.

Proof. This proposition relies on the fact that the differential of the bar construction is defined with the coproduct $\Delta$ of $\overline{\mathcal{B}}(\mathcal{P})=\mathcal{F}^{c}(\Sigma \overline{\mathcal{P}})$ and that the differential of the Koszul complex is also defined with the coproduct on $\mathcal{P}^{i}$. The Koszul dual $\mathcal{P}^{i}$ is a sub-coproperad of $\overline{\mathcal{B}}(\mathcal{P})$, which concludes the proof.

The inclusion $\mathcal{P}^{\mathrm{i}} \hookrightarrow \overline{\mathcal{B}}(\mathcal{P})$ induces a morphism of dg-S-bimodules

$$
L \otimes_{c} \mathcal{P}^{\mathrm{i}} \otimes_{c} R \hookrightarrow \mathcal{B}(L, \mathcal{P}, R) .
$$

7.3.2. Koszul complex and minimal model. We have studied a particular bar construction, namely the augmented bar construction $\mathcal{B}(I, \mathcal{P}, \mathcal{P})=\overline{\mathcal{B}}(\mathcal{P}) \bigotimes_{c} \mathcal{P}$. Here we consider the equivalent chain complex in the framework of Koszul complexes.

Definition (Koszul complex). We call a Koszul complex the chain complex

$$
\mathcal{K}(I, \mathcal{P}, \mathcal{P})=\mathcal{P}^{i} \nabla_{c} \mathcal{P}
$$

(and $\left.\mathcal{K}(\mathcal{P}, \mathcal{P}, I)=\mathcal{P} \otimes_{c} \mathcal{P} \mathrm{i}\right)$.

The differential of this complex is defined by the sum of the morphism $d_{\theta_{r}}$ with the canonical differential $\delta_{\mathcal{P}}$ induced by the one of $\mathcal{P}$. Remark that the morphism $d_{\theta_{r}}$ corresponds to extract one operation $\nu \in \mathcal{P}_{(1)}^{\mathrm{i}}$ of $\mathcal{P}$ i from the top, identify this operation $\nu$ as an operation of $\mathcal{P}_{(1)}$ (since $\mathcal{P}_{(1)}^{\mathrm{i}} \cong \mathcal{P}_{(1)}$ ) and finally compose it in $\mathcal{P}$. This can be graphically resumed by the same kind of diagram as the ones given by J.-L. Loday in [L1] for associative algebras.

The main theorem of this paper is the following criterion. 
Theorem 7.8 (Koszul criterion). Let $\mathcal{P}$ be a weight graded connected dg-properad. The following assertions are equivalent:

(1) The properad $\mathcal{P}$ is a Koszul properad (the inclusion $\mathcal{P}^{i} \hookrightarrow \overline{\mathcal{B}}(\mathcal{P})$ is a quasi-isomorphism).

(2) The Koszul complex $\mathcal{P}^{i} \otimes_{c} \mathcal{P}$ is acyclic.

$\left(2^{\prime}\right)$ The Koszul complex $\mathcal{P} \otimes_{c} \mathcal{P} i$ is acyclic.

(3) The morphism of weight graded dg-properads $\overline{\mathcal{B}}^{c}\left(\mathcal{P}^{i}\right) \rightarrow \mathcal{P}$ is a quasi-isomorphism.

Proof. We have already seen the equivalence (1) $\Longleftrightarrow$ (3) (cf. Theorem 7.6).

By the comparison lemma for quasi-free $\mathcal{P}$-modules (on the right), the assertion (1) is equivalent to the fact that the morphism $\mathcal{P}^{\mathrm{i}} \otimes_{c} \mathcal{P} \rightarrow \overline{\mathcal{B}}(\mathcal{P}) \bigotimes_{c} \mathcal{P}$ is a quasiisomorphism. The acyclicity of the augmented bar construction (Theorem 4.15) shows that the properad $\mathcal{P}$ is Koszul (1) if and only if the Koszul complex $\mathcal{P}$ i $\bigotimes_{c} \mathcal{P}$ is acyclic (2).

We use the comparison lemma for quasi-free $\mathcal{P}$-modules on the left, to prove the equivalence $(1) \Longleftrightarrow\left(2^{\prime}\right)$.

7.4. Koszul duality for props. One can write the same theory for quadratic props. We are going to show how to retrieve Koszul duality for quadratic props defined by connected relations ( $c f$. 2.9) from Koszul duality of properads.

Let $\widetilde{\mathcal{P}}$ be quadratic prop $\widetilde{\mathcal{F}}(V) /(R)$ defined by connected relations $R \subset \mathcal{F}_{(2)}(V) \subset$ $\widetilde{\mathcal{F}}_{(2)}(V)$. Recall from Proposition 2.10 that $\widetilde{\mathcal{P}}=\widetilde{\mathcal{F}}(V) /(R)=\mathcal{S}(\mathcal{F}(V) /(R))$ is isomorphic to the free prop on the properad $\mathcal{P}=\mathcal{F}(V) /(R)$. With the same methods, one can generalize the bar and cobar constructions, the Koszul duals and complexes to props. Denote by $\sim$ the constructions related to props. Since the concatenation of elements of $\widetilde{\mathcal{P}}$ is free, the following diagram is commutative:

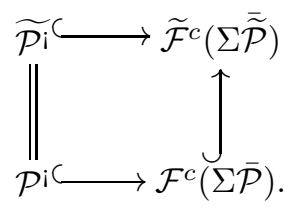

In other words, the Koszul dual coprop $\widetilde{\mathcal{P}}$ is a coproperad isomorphic to the Koszul dual $\mathcal{P}$ i of $\mathcal{P}$. One can also see that the Koszul complex $\widetilde{\mathcal{P}} \otimes \widetilde{\mathcal{P}}$ associated to the prop $\widetilde{\mathcal{P}}$ is isomorphic to $\mathcal{S}\left(\mathcal{P}^{i} \otimes_{c} \mathcal{P}\right)$. Hence, one is acyclic if and only if the other is acyclic. The cobar construction $\widetilde{\mathcal{F}}\left(\Sigma^{-1} \overline{\overline{\mathcal{P}} \mathrm{i}}\right)$ on $\widetilde{\mathcal{P}}$ is isomorphic to $\mathcal{S}\left(\mathcal{F}\left(\Sigma^{-1} \overline{\mathcal{P}} \mathrm{i}\right)\right)=\mathcal{S}\left(\overline{\mathcal{B}}^{c}(\mathcal{P} \mathrm{i})\right.$. Since $\widetilde{\mathcal{P}}=\mathcal{S}(\mathcal{P})$, the cobar construction on $\widetilde{\mathcal{P}}$ i is a resolution of $\widetilde{\mathcal{P}}$ (morphism of props) if and only if the cobar construction on $\mathcal{P} i$ is a resolution of $\mathcal{P}$ (morphism of properads).

Corollary 7.9. Let $\widetilde{\mathcal{P}}$ be a quadratic connected dg-prop defined by connected relations. The following assertions are equivalent.

(1) The Koszul complex $\widetilde{\mathcal{P}} \boldsymbol{\nabla} \widetilde{\mathcal{P}}$ is acyclic.

(2) The morphism of weight graded dg-props $\overline{\overline{\mathcal{B}}^{c}}(\widetilde{\mathcal{P}} i) \rightarrow \widetilde{\mathcal{P}}$ is a quasi-isomorphism. 
Remark. Since the author does not know any type of gebras defined on a quadratic prop with non-confected relations, we are reluctant to write Koszul duality for props in whole generality. The Koszul resolutions for props are based on the free prop which is much bigger than the free properad. For the applications of Koszul duality studied in this paper (for instance notion of $\mathcal{P}$-gebra up to homotopy $c f$. 8.4), the resolution of the underlying properad is enough and contains all the information about the algebraic structure.

7.5. Relation with the Koszul duality theories for associative algebras and for operads. The notion of a Koszul dual of a properad introduced here is a coproperad ( $c f$. 7.1). On the contrary, the Koszul dual of an associative algebra is an algebra in $[\mathrm{Pr}$, and the Koszul dual of an operad is an operad in [GK]. To recover these classical constructions, we consider their linear dual : the Czech dual of $\mathcal{P}^{\mathrm{i}}$.

Definition (Czech dual of an $\mathbb{S}$-bimodule). Let $\mathcal{P}$ be an $\mathbb{S}$-bimodule; we define the Czech dual $\mathcal{P}^{\vee}$ of $\mathcal{P}$ by the $\mathbb{S}$-bimodule $\mathcal{P}^{\vee}:=\bigoplus_{\rho, m, n} \mathcal{P}_{(\rho)}^{\vee}(m, n)$, where

$$
\mathcal{P}_{(\rho)}^{\vee}(m, n):=s g n_{\mathbb{S}_{m}} \otimes_{k} \mathcal{P}_{(\rho)}(m, n)^{*} \otimes_{k} s g n_{\mathbb{S}_{n}} .
$$

The Czech dual is the linear dual twisted by the signature representations.

Lemma 7.10. Let $(\mathcal{C}, \Delta, \varepsilon)$ be a weight graded coproperad such that the dimensions of the modules $\mathcal{C}_{(\rho)}(m, n)$ are finite over $k$, for every $m, n$ and $\rho$. The $\mathbb{S}$-bimodule $\mathcal{C}^{\vee}$ is naturally endowed with a structure of a weight graded properad.

Proof. Denote $\mathcal{P}=\mathcal{C}^{\vee}$. The coproduct $\Delta$ can be decomposed by the weight $\Delta=$ $\bigoplus_{\rho \in \mathbb{N}} \Delta_{(\rho)}$. To define the multiplication $\mu$ on $\mathcal{C}^{\vee}$, we dualize

$$
\Delta_{(\rho)}(m, n): \mathcal{C}_{(\rho)}(m, n) \rightarrow\left(\mathcal{C} \otimes_{c} \mathcal{C}\right)_{(\rho)}(m, n) .
$$

More precisely, we have

$$
\begin{aligned}
& \left.\left(\mathcal{P} \otimes_{c} \mathcal{P}\right)_{(\rho)}(m, n)=\bigoplus_{g \in \mathcal{G}_{c}^{2}(m, n)}\left(\bigotimes_{\substack{\text { vertex of } g \\
\sum \rho_{\nu}=\rho}} \mathcal{C}_{\left(\rho_{\nu}\right)}^{\vee}(|\operatorname{Out}(\nu)|,|\operatorname{In}(\nu)|)\right)\right]_{\approx} \\
& \left.=\bigoplus_{g \in \mathcal{G}_{c}^{2}(m, n)}\left(\left(\bigotimes_{\substack{\nu \text { vertex of } g \\
\Sigma \rho_{\nu}=\rho}} \mathcal{C}_{\left(\rho_{\nu}\right)}(|\operatorname{Out}(\nu)|,|\operatorname{In}(\nu)|)\right)\right)_{\approx}\right)^{\vee},
\end{aligned}
$$

using the finite dimensional hypothesis of the $\mathcal{C}_{(\rho)}(m, n)$ and identifying the invariants with the coinvariants (we work over a field $k$ of characteristic 0 ). Hence, we define the composition $\mu_{(\rho)}$ by the formula:

$$
\begin{aligned}
\left(\mathcal{P} \otimes_{c} \mathcal{P}\right)_{(\rho)}(m, n) & =\bigoplus_{g \in \mathcal{G}_{c}^{2}(m, n)}\left(\left(\bigotimes_{\substack{\text { vertex of } g \\
\sum \rho_{\nu}=\rho}} \mathcal{C}_{\left(\rho_{\nu}\right)}(\mid \text { Out }(\nu)|,| \operatorname{In}(\nu) \mid)\right)\right)^{\vee} \\
& \longrightarrow\left(\bigoplus_{g \in \mathcal{G}_{c}^{2}(m, n)}\left(\bigotimes_{\substack{\nu \text { vertex of } g \\
\Sigma \rho_{\nu}=\rho}} \mathcal{C}_{\left(\rho_{\nu}\right)}(\mid \text { Out }(\nu)|,| \operatorname{In}(\nu) \mid)\right)\right)^{\vee} \\
& =\left(\mathcal{C} \otimes_{\mathcal{C}} \mathcal{C}\right)_{(\rho)}^{\vee}(m, n) \stackrel{{ }^{t} \Delta_{(\rho)}}{\longrightarrow} \mathcal{C}_{(\rho)}^{\vee}(m, n)=\mathcal{P}_{(\rho)}(m, n) .
\end{aligned}
$$


The coassociativity of the coproduct $\Delta$ induces the associativity of the product $\mu$. Also, the counit of $\mathcal{C} \varepsilon: \mathcal{C} \rightarrow I$ gives, after passage to the dual, the unit of $\mathcal{P}$ : $\varepsilon: I \rightarrow \mathcal{P}$.

Proposition 7.11. Let $\mathcal{P}$ be a weight graded properad (for instance a quadratic properad). Denote $V=\mathcal{P}^{(1)}$, the $\mathbb{S}$-bimodule generated by the elements of weight 1 of $\mathcal{P}$.

If there exist two integers $M$ and $N$ such that $V(m, n)=0$ for $m>M$ or $n>N$ and if the dimension of every module $V(m, n)$ is finite on $k$, then the cobar construction $\overline{\mathcal{B}}^{c}(\mathcal{P})$ on $\mathcal{P}$ and the Koszul dual $\mathcal{P}^{i}$ verify the hypothesis of the preceding lemma.

Proof. Since $\mathcal{P}$ i is a weight graded sub-coproperad of the cobar construction $\overline{\mathcal{B}}^{c}(\mathcal{P})$, it is enough to show that the dimension of the modules $\overline{\mathcal{B}}^{c}(\mathcal{P})_{(\rho)}(m, n)$ is finite.

We have seen that $\overline{\mathcal{B}}^{c}(\mathcal{P})_{(\rho)}(m, n)=\mathcal{F}_{(\rho)}^{c}(V)(m, n)$. In the case where $V$ verifies the hypotheses of the proposition, this module is given by a sum indexed by the set of graphs with $\rho$ vertices and such that each vertex has at most $N$ inputs and $M$ outputs. Since this set is finite, the dimension of the modules $V(m, n)$ is finite.

Corollary 7.12. For every quadratic properad generated by an $\mathbb{S}$-bimodule $V$ such that the sum of the dimensions $\sum_{m, n} \operatorname{dim}_{k} V(m, n)$ is finite, the Czech dual $\mathcal{P}^{\vee}$ of the Koszul dual of $\mathcal{P}$ is endowed with a natural structure of properad .

Moreover, if $\mathcal{P}=\mathcal{F}(V) /(R)$, then the properad $\mathcal{P}^{\vee}$ is quadratic and $\mathcal{P}^{\vee}=$ $\mathcal{F}(\Sigma V) /\left(\Sigma^{2} R^{\perp}\right)$. We follow the classical notation and denote this properad $\mathcal{P}^{\text {! }}$. $\Sigma^{2} R$.

One can notice that the properad $\mathcal{P}^{!}$is determined by $\mathcal{P}_{(1)}^{\mathrm{i}}=\Sigma V$ and $\mathcal{P}_{(2)}^{\mathrm{i}}=$

Recall that in the case of quadratic algebra $A=T(V) /(R)$, S. Priddy defined the dual algebra $A^{!}$by $T\left(V^{*}\right) /\left(R^{\perp}\right)$, when $V$ is finite dimensional. In the same way, V. Ginzburg and M. M. Kapranov define the dual of a quadratic operad $\mathcal{P}=\mathcal{F}(V) /(R)$ by $\mathcal{P}^{!}=\mathcal{F}\left(V^{\vee}\right) /\left(R^{\perp}\right)$, when $V$ is finite dimensional.

In the finite dimensional case, the conceptual definitions of the dual objects given here correspond to the classical definitions up to suspension ( $c f$. [BGS] and [Fr]). In particular, the dual $A_{(n)}^{\mathrm{i}}$ of an algebra $A$ is isomorphic to $\Sigma^{n} A_{n}^{!^{*}}$, and the dual $\mathcal{P}_{(n)}^{i}$ of an operad $\mathcal{P}$ is isomorphic to $\Sigma^{n} \mathcal{P}_{n}{ }_{n}^{\vee}$.

When $\mathcal{P}$ is an algebra, the Koszul complex and the Koszul resolution, coming from the cobar construction, introduced here correspond to the ones given by $[\mathrm{Pr}$. When $\mathcal{P}$ is an operad, they correspond to the ones given by [GK].

\section{Examples and $\mathcal{P}$-gebra up TO homotopy}

The last difficulty is to be able to prove that the Koszul complex of a properad is acyclic. We consider a large class of properads (properads defined by a replacement rule), and we show that they are Koszul properads. The examples of the properad $\mathcal{B} i \mathcal{L}$ ie of Lie bialgebras and the properad $\varepsilon \mathcal{B} i$ of infinitesimal Hopf bialgebra fall into this case. This method is a generalization to properads of works of T. Fox, M. Markl [FM], M. Markl [Ma3 and W. L. Gan [G]. We define the notion of $\mathcal{P}$-gebra up to homotopy and give the examples of several $\mathcal{P}$-gebras up to homotopy. 
8.1. Replacement rule. Let $\mathcal{P}$ be a quadratic properad of the form

$$
\mathcal{P}=\mathcal{F}(V, W) /(R \oplus D \oplus S),
$$

where $R \subset \mathcal{F}_{(2)}(V), S \subset \mathcal{F}_{(2)}(W)$ and

$$
D \subset(I \oplus \underbrace{W}_{1}) \otimes_{c}(I \oplus \underbrace{V}_{1}) \bigoplus(I \oplus \underbrace{V}_{1}) \otimes_{c}(I \oplus \underbrace{W}_{1}) .
$$

The two pairs of $\mathbb{S}$-bimodules $(V, R)$ and $(W, S)$ generate two properads denoted as $\mathcal{A}=\mathcal{F}(V) /(R)$ and $\mathcal{B}=\mathcal{F}(W) /(S)$.

Definition (Replacement rule). Let $\lambda$ be a morphism of $\mathbb{S}$-bimodules

$$
\lambda:(I \oplus \underbrace{W}_{1}) \otimes_{c}(I \oplus \underbrace{V}_{1}) \rightarrow(I \oplus \underbrace{V}_{1}) \otimes_{c}(I \oplus \underbrace{W}_{1})
$$

such that the $\mathbb{S}$-bimodule $D$ is defined by the image

$(i d,-\lambda):(I \oplus \underbrace{W}_{1}) \otimes_{c}(I \oplus \underbrace{V}_{1}) \rightarrow(I \oplus \underbrace{W}_{1}) \otimes_{c}(I \oplus \underbrace{V}_{1}) \bigoplus(I \oplus \underbrace{V}_{1}) \bigotimes_{c}(I \oplus \underbrace{W}_{1})$.

We call $\lambda$ a replacement rule and denote $D$ by $D_{\lambda}$ if the two following morphisms are injective:

$$
\left\{\begin{array}{l}
\underbrace{\mathcal{A}}_{1} \otimes_{c} \underbrace{\mathcal{B}}_{2} \rightarrow \mathcal{P}, \\
\underbrace{\mathcal{A}}_{2} \otimes_{c} \underbrace{\mathcal{B}}_{1} \rightarrow \mathcal{P} .
\end{array}\right.
$$

Remark. The last condition must be seen as a coherence axiom. It ensures that the natural morphism $\mathcal{A} \otimes \mathcal{B} \rightarrow \mathcal{P}$ is injective (cf. $[\overline{\mathrm{FM}})$.

The examples treated here are of this form. They come from the same kind of "distributive law" between two operads described in [FM] and in [Ma3].

Definition (Reversed $\mathbb{S}$-bimodule). Let $\mathcal{P}$ be an $\mathbb{S}$-bimodule. We define its reversed S-bimodule by

$$
\mathcal{P}^{o p}(m, n):=\mathcal{P}(n, m) .
$$

The action to take the reversed $\mathbb{S}$-bimodule $\mathcal{P}^{o p}$ corresponds to inverse the direction of the operations of $\mathcal{P}$. Let $\mathcal{P}$ and $\mathcal{Q}$ be two $\mathbb{S}$-bimodules; we have

$$
\left(\mathcal{P} \otimes_{c} \mathcal{Q}\right)^{o p}=\mathcal{Q}^{o p} \bigotimes_{c} \mathcal{P}^{o p} .
$$

When the $\mathbb{S}$-bimodule $\mathcal{P}$ is endowed with a structure of properad, the reversed $\mathbb{S}$-bimodule $\mathcal{P}^{o p}$ is also a properad.

The properads treated $(\mathcal{B} i \mathcal{L} i e$ and $\varepsilon \mathcal{B} i)$ are generated by operations ( $n$ inputs and one output) and cooperations (one input and $m$ outputs). One can write them as $\mathcal{F}(V \oplus W) /\left(R \oplus D_{\lambda} \oplus S\right)$, where $V$ represents the generating operations $(V(m, n)=0$ for $m>1)$ and where $W$ represents the generating cooperations $(W(m, n)=0$ for $n>1)$. The properad $\mathcal{A}=\mathcal{F}(V) /(R)$ is an operad, and the properad $\mathcal{B}^{o p}=\mathcal{F}\left(W^{o p}\right) /\left(S^{o p}\right)$ is an operad too. In this case, the replacement rule has the following form:

$$
\lambda: W \otimes_{k} V \rightarrow(I \oplus \underbrace{V}_{1}) \bigotimes_{c}(I \oplus \underbrace{W}_{1}) .
$$


For the properad $\mathcal{B}$ i $\mathcal{L}$ ie associated to Lie bialgebras ( $c f$. 2.9), one has $\mathcal{A}=\mathcal{B}^{o p}=$ $\mathcal{L} i e$ and the replacement rule $\lambda$ is given by

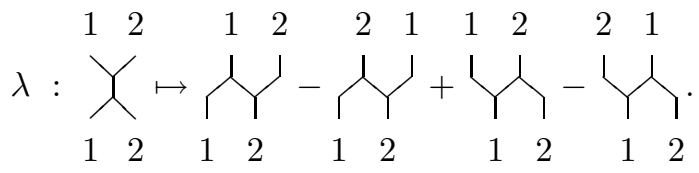

In the case of the properad $\varepsilon \mathcal{B} i$ of an infinitesimal Hopf bialgebra ( $c f$. 2.9), the operads $\mathcal{A}$ and $\mathcal{B}^{o p}$ are equal to the operad $\mathcal{A} s$ of associative algebras, and the replacement rule is given by

$$
\lambda: X \mapsto \gamma+Y .
$$

A replacement rule allows us to vertically permutate operations and cooperations.

Lemma 8.1. Every properad of the form $\mathcal{P}=\mathcal{F}(V, W) /\left(R \oplus D_{\lambda} \oplus S\right)$ defined by a replacement rule $\lambda$ is isomorphic to the $\mathbb{S}$-bimodule

$$
\mathcal{P} \cong \mathcal{A} \otimes_{c} \mathcal{B} \text {. }
$$

Proof. As we have seen in the previous remark, the last condition defining a replacement rule ensures that the natural morphism $\mathcal{A} \otimes_{c} \mathcal{B} \rightarrow \mathcal{P}$ is injective.

To show that it is also surjective, we choose a representative in $\mathcal{F}(V \oplus W)$ of every element of $\mathcal{P}$. It can be written as a finite sum of graphs indexed by operations of $V$ and $W$. For every graph, we fix each vertex on a level and we vertically permutate elements of $V$ and elements of $W$ with the replacement rule to finally have all the elements of $V$ under the ones of $W$. Therefore, we get that an element of $\mathcal{F}(V) \bigotimes_{c} \mathcal{F}(W)$ which projected in $\mathcal{A} \bigotimes_{c} \mathcal{B}$ gives the wanted element.

In the two previous examples, this lemma shows that we can write every element of $\mathcal{P}$ as a sum of elements of $\mathcal{A} \nabla_{c} \mathcal{B}$. This corresponds to put the cooperations of $\mathcal{B}$ at the top and the operations of $\mathcal{A}$ under. In the case of Lie bialgebras, this result has been proved by B. Enriquez and P. Etingov in EE] (Section 6.4).

\subsection{Koszul dual of a properad defined by a replacement rule.}

Proposition 8.2. Let $\mathcal{P}$ be a properad of the form $\mathcal{P}=\mathcal{F}(V, W) /\left(R \oplus D_{\lambda} \oplus S\right)$ defined by a replacement rule $\lambda$ and such that the sum of the dimensions of $V$ and $W$ on $k, \sum_{m, n} \operatorname{dim}_{k}(V \oplus W)(m, n)$, is finite.

The dual properad is given by

$$
\mathcal{P}^{!}=\mathcal{F}\left(\Sigma W^{\vee} \oplus \Sigma V^{\vee}\right) /\left(\Sigma^{2} S^{\perp} \oplus \Sigma^{2} D_{t_{\lambda}} \oplus \Sigma^{2} R^{\perp}\right),
$$

where the replacement rule is ${ }^{t} \lambda$. Also, the dual coproperad is isomorphic to the S-bimodules

$$
\mathcal{P}^{i} \cong \mathcal{B}^{i} \nabla_{c} \mathcal{A}^{i} .
$$

Proof. Corollary 7.12 shows that $\mathcal{P}^{!^{\vee}} \cong \mathcal{P}^{\mathrm{i}}$.

Denote $R^{\perp}$ as the orthogonal of $R$ in $\mathcal{F}_{(2)}\left(V^{\vee}\right), S^{\perp}$ the orthogonal of $S$ in $\mathcal{F}_{(2)}\left(W^{\vee}\right)$ and $D_{\lambda}^{\perp}$ the orthogonal of $D_{\lambda}$ in $(I \oplus \underbrace{W^{\vee}}_{1}) \bigotimes_{c}(I \oplus \underbrace{V^{\vee}}_{1}) \oplus(I \oplus \underbrace{V^{\vee}}_{1}) \bigotimes_{c}$

$(I \oplus \underbrace{W^{\vee}}_{1})$. Therefore, the dual properad $\mathcal{P}^{!}$is given by

$$
\mathcal{P}^{!}=\mathcal{F}\left(\Sigma V^{\vee} \oplus \Sigma W^{\vee}\right) /\left(\Sigma^{2} R^{\perp} \oplus \Sigma^{2} D_{\lambda}^{\perp} \oplus \Sigma^{2} S^{\perp}\right) .
$$


It remains to see that an $\mathbb{S}$-bimodule $D_{\lambda}^{\perp}$ is isomorphic to the image of the morphism

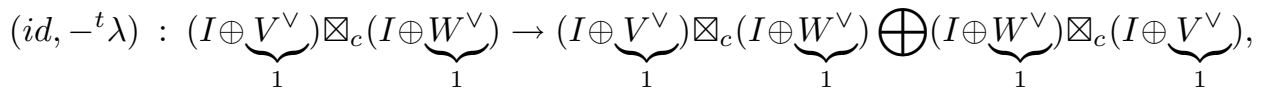

which means that $D_{\lambda}^{\perp}=D^{{ } \lambda}$.

Applying the previous lemma to the properad $\mathcal{P}^{!}$, we get $\mathcal{P}^{!} \cong B^{!} \bigotimes_{c} A^{!}$and $\mathcal{P}^{i} \cong \mathcal{B}^{i} \otimes_{c} \mathcal{A}^{i}$ by Czech duality.

The two examples given by the properads $\mathcal{B} i \mathcal{L} i e$ and $\varepsilon \mathcal{B} i$ of Lie bialgebras and infinitesimal Hopf bialgebras verify the hypotheses of the proposition. They are generated by a finite number of operations and cooperations.

Since the composition of the form $\$ does not appear in their definition, we must have it in the relations of the Koszul duals.

\section{Corollary 8.3.}

(1) The Koszul dual properad $\mathcal{B} i \mathcal{L} i e^{!}$of the properad of Lie bialgebras is given by

$$
\mathcal{B} i \mathcal{L} i e^{!}=\mathcal{F}(V) /(R)
$$

where $V=Y_{1}^{1} \overbrace{2}^{2}$, that's to say, a commutative operation and a cocommutative cooperation, and

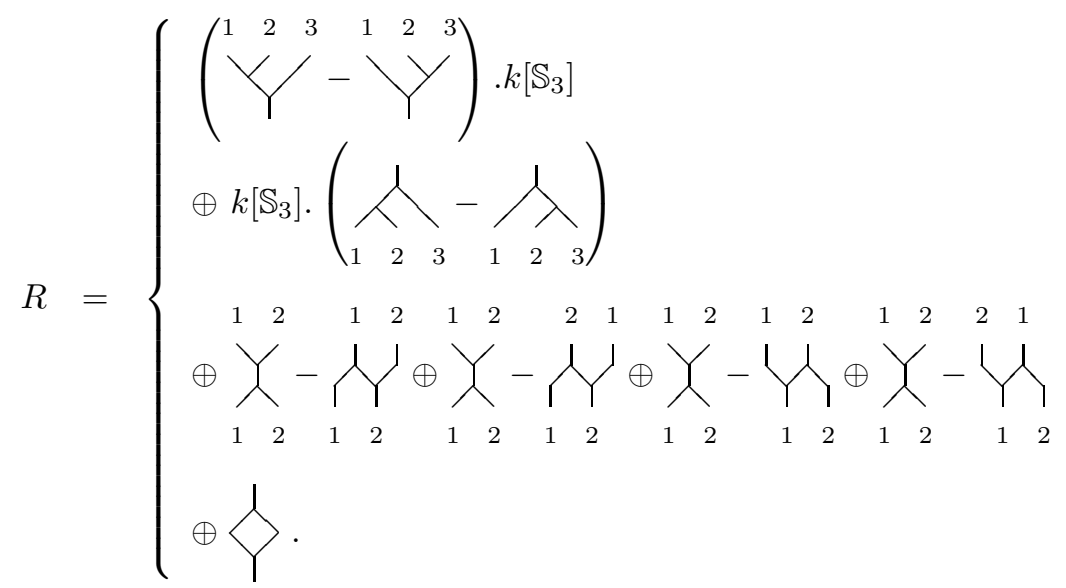

It corresponds to the dioperad of non-unitary Frobenius algebras. As an $\mathbb{S}$-bimodule, it is isomorphic to $\mathcal{B} i \mathcal{L} i e^{!}(m, n)=k$, with trivial actions of $\mathbb{S}_{m}$ and $\mathbb{S}_{n}$.

(2) The Koszul dual properad $\varepsilon \mathcal{B} i^{!}$of the properad of infinitesimal Hopf algebras is given by

$$
\varepsilon \mathcal{B} i^{!}=\mathcal{F}(V) /(R),
$$




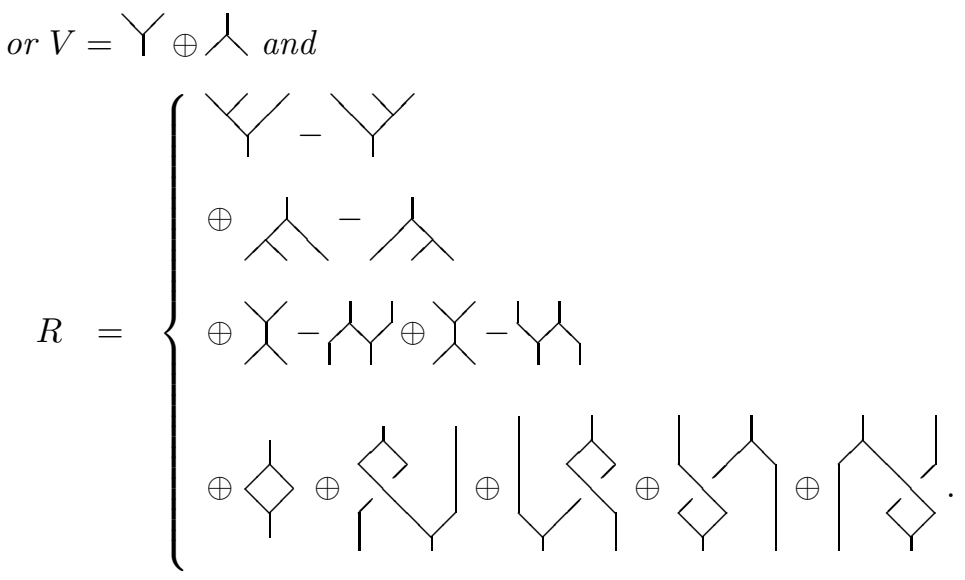

It is isomorphic, as an $\mathbb{S}$-bimodule, to

$$
\varepsilon \mathcal{B} i^{!}(m, n)=k\left[\mathbb{S}_{m}\right] \otimes_{k} k\left[\mathbb{S}_{n}\right] .
$$

\subsection{Koszul complex of a properad defined by a replacement rule.}

Proposition 8.4. Let $\mathcal{P}$ be a properad of the form $\mathcal{P}=\mathcal{F}(V, W) /\left(R \oplus D_{\lambda} \oplus S\right)$ defined by a distributive law $\lambda$ and such that the sum of the dimensions of $V$ and $W$ on $k, \sum_{m, n} \operatorname{dim}_{k}(V \oplus W)(m, n)$, is finite. We define the two properads $\mathcal{A}$ and $\mathcal{B}$ by $\mathcal{A}=\mathcal{F}(V) /(R)$ and $\mathcal{B}=\mathcal{F}(W) /(S)$. We suppose that $\mathcal{B}$ is a properad concentrated in homological degree 0 . If $\mathcal{A}$ and $\mathcal{B}$ are Koszul properads, then $\mathcal{P}$ is a Koszul properad too.

Proof. Lemma 8.1 and Proposition 8.2 show that the Koszul complex of $\mathcal{P}$ is of the following form:

$$
\mathcal{P}^{\mathrm{i}} \nabla_{c} \mathcal{P}=\left(\mathcal{B}^{\mathrm{i}} \otimes_{c} \mathcal{A}^{\mathrm{i}}\right) \otimes_{c}\left(\mathcal{A} \otimes_{c} \mathcal{B}\right)=\mathcal{B}^{\mathrm{i}} \otimes_{c}\left(\mathcal{A}^{\mathrm{i}} \nabla_{c} \mathcal{A}\right) \otimes_{c} \mathcal{B}
$$

We introduce the following filtration of the Koszul complex $F_{n}\left(\mathcal{P} i \otimes_{c} \mathcal{P}\right)$ which corresponds to the sub-S-bimodule of $\mathcal{B}^{i} \nabla_{c}\left(\mathcal{A}^{i} \nabla_{c} \mathcal{A}\right) \bigotimes_{c} \mathcal{B}$ generated by 4 -level graphs where the sum of the weight of the elements of $\mathcal{B}^{i}$ on the fourth level is less than $n$. This filtration is stable under the differential of the Koszul complex. Therefore, it induces a spectral sequence denoted as $E_{p, q}^{*}$. The first term of this spectral sequence $E_{p, q}^{0}$ is isomorphic to the sub-S-bimodule of $\mathcal{B}^{i} \nabla_{c}\left(\mathcal{A}^{i} \nabla_{c} \mathcal{A}\right) \bigotimes_{c} \mathcal{B}$ composed by elements of homological degree $p+q$ and such that the sum of the weight of the elements of $\mathcal{B}^{i}$ on the fourth level is equal to $p$. Since $\mathcal{B}$ is concentrated in homological degree 0 , we have $E_{p, q}^{0}=\underbrace{\mathcal{B}^{\mathrm{i}}}_{p} \nabla_{c} \underbrace{\left(\mathcal{A}^{\mathrm{i}} \otimes_{c} \mathcal{A}\right)}_{q} \otimes_{c} \mathcal{B}$. The differential $d_{0}$ is the differential of the Koszul complex of $\mathcal{A}$. Since $\mathcal{A}$ is a Koszul properad, we get $E_{p, q}^{1}=0$ if $q \neq 0$ and $E_{p, 0}^{1}=\underbrace{B^{\mathrm{i}}}_{p} \otimes_{c} B$. The differential $d_{1}$ is the differential of the Koszul complex of $\mathcal{B}$. Once again, since $\mathcal{B}$ is a Koszul properad, we have

$$
E_{p, q}^{2}= \begin{cases}I & \text { if } p=q=0 \\ 0 & \text { otherwise }\end{cases}
$$

The filtration is exhaustive and bounded from below. Therefore, we can apply the classical theorem of convergence of spectral sequences ( $c f$. [W] 5.5.1) which gives that the spectral sequences converge to the homology of the Koszul complex of $\mathcal{P}$. This complex is acyclic and the properad $\mathcal{P}$ is Koszul. 
Corollary 8.5. The properads $\mathcal{B} i \mathcal{L}$ ie of Lie bialgebras and $\varepsilon \mathcal{B} i$ of infinitesimal Hopf algebras.

Proof. In the case of $\mathcal{B} i \mathcal{L} i e$, the properad $\mathcal{A}$ is the Koszul operad of Lie algebras $\mathcal{L} i e$ and the properad $\mathcal{B}$ is the reversed properad of the Koszul operad $\mathcal{L} i e, \mathcal{B}=\mathcal{L} i e^{o p}$, which is also a Koszul properad.

In the case of $\varepsilon \mathcal{B} i$, the properad $\mathcal{A}$ is the Koszul operad $\mathcal{A} s$ of associative algebras and the properad $\mathcal{B}$ is the reversed properad of the Koszul operad $\mathcal{A} s, \mathcal{B}=\mathcal{A} s^{o p}$, which is also a Koszul properad.

Application: The cobar construction of the coproperad $\mathcal{B} i \mathcal{L} i e^{i}$ is a resolution of the properad $\mathcal{B}$ iLie. We can interpret this homological result in terms of graph cohomology. Since the Koszul dual properad of $\mathcal{B} i \mathcal{L} i e$ is a dioperad, the differential of the cobar construction of $\mathcal{B} i \mathcal{L} i e^{i}$ is equal to the boundary map defined by M. Kontsevich in the context of graph cohomology (vertex expansion). Therefore, we find the results of M. Markl and A.A. Voronov in [MV] : the cohomology of the directed connected commutative graphs is equal to the properad $\mathcal{B} i \mathcal{L} i e$, and the cohomology of the directed connected ribbon graphs is equal to the properad $\varepsilon \mathcal{B} i$.

\section{4. $\mathcal{P}$-gebra up to homotopy.}

Definition ( $\mathcal{P}$-gebra up to homotopy). When $\mathcal{P}$ is a Koszul properad, we call $\mathcal{P}$ gebra up to homotopy any gebra on $\overline{\mathcal{B}}^{c}(\mathcal{P} \mathrm{i})$. We denote the category of $\mathcal{P}$-gebras up to homotopy by $\mathcal{P}_{\infty}$-gebras.

This notion generalizes to gebras the notion of algebra up to homotopy (on an operad). For instance, we have the notion of Lie bialgebras up to homotopy and the notion of infinitesimal Hopf algebras up to homotopy.

\section{Poincaré Series}

Here we generalize the Poincaré series of quadratic associative algebras (cf. J.-L. Loday [L1]) and binary quadratic operads (cf. V. Ginzburg and M.M. Kapranov [GK] ) to properads. When a properad $\mathcal{P}$ is Koszul, we show a functional equation between the Poincaré series of $\mathcal{P}$ and $\mathcal{P}^{i}$.

9.1. Poincaré series for Koszul properads. Let $\mathcal{P}$ be a weight graded connected dg-properad. Denote by $\mathcal{K}$ its Koszul complex $\mathcal{K}(I, \mathcal{P}, \mathcal{P})=\mathcal{P}^{i} \otimes_{c} \mathcal{P}$. This complex is the directed sum of its sub-complexes indexed by the global weight $\mathcal{K}=\bigoplus_{m, n, d \geq 0} \mathcal{K}_{(d)}(m, n)$, where $\mathcal{K}_{(d)}(m, n)$ has the following form :

$$
\begin{aligned}
0 & \rightarrow \mathcal{P}_{(d)}^{\mathrm{i}}(m, n) \rightarrow \underbrace{\mathcal{P}^{\mathrm{i}}}_{(d-1)} \otimes_{c} \underbrace{\mathcal{P}}_{(1)}(m, n) \rightarrow \cdots \rightarrow \underbrace{\mathcal{P}}_{(1)} \bigotimes_{c} \underbrace{\mathcal{P}}_{(d-1)}(m, n) \\
& \rightarrow \mathcal{P}_{(d)}(m, n) \rightarrow 0 .
\end{aligned}
$$

The main theorem of this paper (Theorem 7.8 ) asserts that a properad $\mathcal{P}$ is Koszul if and only if each chain complex $\mathcal{K}_{(d)}(m, n)$ is acyclic for $d>0$. In this case, we know from Corollary 7.5 that the properad $\mathcal{P}$ is quadratic. Suppose that $\mathcal{P}$ is a quadratic properad generated by an $\mathbb{S}$-module $V$ such that the total dimension of $\bigoplus_{m, n \in \mathbb{N}^{*}} V(m, n)$ is finite. In Proposition 7.11 we have seen that each module 
$\mathcal{F}_{(d)}(V)(m, n)$ has finite dimension. Therefore, the dimension of each $\mathcal{P}_{(d)}(m, n)$ and $\mathcal{P}_{(d)}^{\mathrm{i}}(m, n)$ is finite. The Euler-Poincaré characteristic of $\mathcal{K}_{(d)}(m, n)$ is null

$$
\sum_{k=0}^{d}(-1)^{k} \operatorname{dim}(\underbrace{\mathcal{P} \mathrm{i}}_{(k)} \otimes_{c} \underbrace{\mathcal{P}}_{(d-k)})(m, n)=0 .
$$

The Euler-Poincaré characteristic is given by the following formula:

$$
\begin{aligned}
& \sum_{k=0}^{d}(-1)^{k} \operatorname{dim}(\underbrace{\mathcal{P} \mathbf{i}}_{(k)} \otimes_{c} \underbrace{\mathcal{P}}_{(d-k)})(m, n) \\
& =\sum_{\Xi} \sharp \mathcal{S}_{c}^{\bar{k}, \bar{j}} \frac{n !}{\bar{\imath} ! \bar{\jmath} !} \frac{m !}{\bar{k} ! \bar{l} !} \operatorname{dim} \mathcal{P}_{\left(o_{1}\right)}^{!}\left(l_{1}, k_{1}\right) \ldots \operatorname{dim} \mathcal{P}_{\left(o_{b}\right)}^{\mathrm{i}}\left(l_{b}, k_{b}\right) \\
& \quad \times \operatorname{dim} \mathcal{P}_{\left(q_{1}\right)}\left(j_{1}, i_{1}\right) \ldots \operatorname{dim} \mathcal{P}_{\left(q_{a}\right)}\left(j_{a}, i_{a}\right),
\end{aligned}
$$

where the sum $\Xi$ runs over the $n$-tuples $\bar{\imath}, \bar{\jmath}, \bar{k}, \bar{l}, \bar{o}$ and $\bar{q}$ such that $|\bar{\imath}|=n,|\bar{\jmath}|=|\bar{k}|$, $|\bar{l}|=m,|\bar{o}|=k$ and $|\bar{q}|=d-k$.

Definition (Poincaré series associated to an $\mathbb{S}$-bimodule). To a weight graded $\mathbb{S}$ bimodule $\mathcal{P}$, we associate the Poincaré series defined by

$$
f_{\mathcal{P}}(y, x, z):=\sum_{\substack{m, n \geq 1 \\ d \geq 0}} \frac{\operatorname{dim} \mathcal{P}_{(d)}(m, n)}{m ! n !} y^{m} x^{n} z^{d},
$$

when the dimension of every $k$-modules $\mathcal{P}_{(d)}(m, n)$ is finite.

We define the function $\Psi$ by the formula

$\Psi\left(g(y, X, z), f\left(Y, x, z^{\prime}\right)\right):=\sum_{\Xi^{\prime}} \sharp S_{c}^{\bar{k}, \bar{j}} \prod_{\beta=1}^{b} \frac{1}{k_{\beta} !} \frac{\partial^{k_{\beta}} g}{\partial X^{k_{\beta}}}(y, 0, z) \prod_{\alpha=1}^{a} \frac{1}{j_{\alpha} !} \frac{\partial^{j_{\alpha}} f}{\partial Y^{j_{\alpha}}}\left(0, x, z^{\prime}\right)$,

where the sum $\Xi^{\prime}$ runs over the $n$-tuples $\bar{k}$ and $\bar{\jmath}$ such that $|\bar{k}|=|\bar{\jmath}|$.

Theorem 9.1. Every Koszul properad $\mathcal{P}$ generated by an $\mathbb{S}$-module of global finite dimension verifies the following equation:

$$
\Psi\left(f_{\mathcal{P}^{i}}(y, X,-z), f_{\mathcal{P}}(Y, x, z)\right)=x y .
$$

Proof. We have

$$
\begin{aligned}
& \Psi\left(f_{\mathcal{P i}}(y, X,-z), f_{\mathcal{P}}(Y, x, z)\right) \\
& =\sum_{\Xi^{\prime}} \sharp S_{c}^{\bar{k}, \bar{j}} \prod_{\beta=1}^{b} \frac{1}{k_{\beta} !} \frac{\partial_{\beta}^{k} f_{\mathcal{P} \mathrm{i}}}{\partial X^{k_{\beta}}}(y, 0,-z) \prod_{\alpha=1}^{a} \frac{1}{j_{\alpha} !} \frac{\partial^{j_{\alpha}} f_{\mathcal{P}}}{\partial Y^{j_{\alpha}}}(0, x, z) \\
& \times \sum_{\substack{m_{n}, n \geq 1 \\
d \geq 0}}(\underbrace{\sum_{k=0}^{d}(-1)^{k}\left(\sum_{\Xi} \sharp S_{c}^{\bar{k}, \bar{j}} \prod_{\beta=1}^{b} \frac{d i m \mathcal{P}_{q_{\beta}}^{i}\left(l_{\beta}, k_{\beta}\right)}{l_{\beta} ! k_{\beta} !} \prod_{\alpha=1}^{a} \frac{\operatorname{dim} \mathcal{P}_{o_{\alpha}}\left(j_{\alpha}, i_{\alpha}\right)}{j_{\alpha} ! i_{\alpha} !}\right)}_{=0 \quad \text { for }}) y^{m} x^{n} z^{d} \\
& =x y .
\end{aligned}
$$


Remark. Since the Koszul complex $\mathcal{K}(I, \mathcal{P}, \mathcal{P})$ is acyclic if and only if the Koszul complex $\mathcal{K}(\mathcal{P}, \mathcal{P}, I)$ is acyclic, we also have the symmetric relation

$$
\Psi\left(f_{\mathcal{P}}(y, X,-z), f_{\mathcal{P}_{\mathrm{i}}}(Y, x, z)\right)=x y .
$$

9.2. Poincaré series for associative Koszul algebras. When the quadratic properad $\mathcal{P}$ is a quadratic algebra $A$, its Poincaré series equals $\sum_{d \geq 0} \operatorname{dim}\left(A_{(d)}\right) z^{d} x y$. If we denote

$$
f_{A}(z):=\sum_{d \geq 0} \operatorname{dim}\left(A_{(d)}\right) z^{d},
$$

then the functional equation of the previous theorem is equivalent to the well known relation ( $c f$. [L1])

$$
f_{A}(x) \cdot f_{A^{i}}(-x)=1 .
$$

9.3. Poincaré series for Koszul operads. When the quadratic properad $\mathcal{P}$ is a quadratic operad, its Poincaré series is equal to

$$
\sum_{d \geq 0, n \geq 1} \frac{\operatorname{dim} \mathcal{P}_{(d)}(n)}{n !} x^{n} y z^{d}
$$

We denote

$$
f_{\mathcal{P}}(x, z):=\sum_{d \geq 0, n \geq 1} \frac{\operatorname{dim} \mathcal{P}_{(d)}(n)}{n !} x^{n} z^{d} .
$$

Corollary 9.2. The Poincaré series of a Koszul operad $\mathcal{P}$ verifies the relation

$$
f_{\mathcal{P}^{i}}\left(f_{\mathcal{P}}(x, z),-z\right)=x .
$$

In the binary case (when $V=V(2)$ ), we have $\mathcal{P}_{(n-1)}=\mathcal{P}(n)$ and

$$
f_{\mathcal{P}}(x, z)=\sum_{n \geq 1} \frac{\operatorname{dim} \mathcal{P}(n)}{n !} x^{n} z^{n-1} .
$$

We denote

$$
f_{\mathcal{P}}(x):=\sum_{n \geq 1}(-1)^{n} \frac{\operatorname{dim} \mathcal{P}(n)}{n !} x^{n},
$$

and if $\mathcal{P}$ is Koszul, we have that the preceding theorem gives the well known relation (cf. [22 Appendix B)

$$
f_{\mathcal{P} i}\left(f_{\mathcal{P}}(x)\right)=x .
$$

Example. Consider the free operad $\mathcal{P}=\mathcal{F}(V)$ generated by the $\mathbb{S}$-module $V=$ $k\{Y, Y, \ldots\}$ composed by one $n$-ary operation for every $n$. Since it is a free operad, it is a Koszul operad. Whereas the total dimension of $V$ is infinite, the dimension of every $\mathcal{F}(V)(n)$ is finite. Therefore, we can consider the Euler-Poincaré characteristic of the Koszul complex of $\mathcal{P}$. Since $\mathcal{P}^{i}=k \oplus V$, we have

$$
f_{\mathcal{P i}}(x, z)=\sum_{d \geq 0, n \geq 1} \operatorname{dim}_{(d)}^{\mathrm{i}}(n) x^{n} z^{d}=x+\sum_{n \geq 2} x^{n} z=x+z \frac{x^{2}}{1-x} .
$$

Also, Corollary 9.2 gives the following equation:

$$
(z+1) f_{\mathcal{P}}^{2}(x, z)-(1+x) f_{\mathcal{P}}(x, z)+x=0 .
$$

Let $P_{n}(z)$ be the Poincaré polynomial of the Stasheff polytope of dimension $n$, also called the associahedra and denoted as $K^{n}$ or $K_{n+2}$. This polynomial is equal to $P_{n}(z):=\sum_{k=0}^{n} \sharp C e l_{k}^{n} . z^{k}$, where $C e l_{k}^{n}$ represents the set of the cells of 
dimension $k$ of the polytope of dimension $n$. Denote $f_{K}(x, z)=\sum_{n \geq 0} P_{n}(z) x^{n}$ as the generating series.

The cells of dimension $k$ of $K^{n}$ are indexed by planar trees with $n+2$ leaves and $n+1-k$ vertices. This bijection implies that $\sharp C e l_{k}^{n}=\operatorname{dim}_{n+1-k}(n+2)$, which gives

$$
\begin{aligned}
f_{\mathcal{P}}(x, z) & =x+\sum_{n \geq 2} \sum_{k=1}^{n-1} \operatorname{dim}_{k}^{n} z^{k} x^{n} \\
& =x+\sum_{n \geq 2}\left(\sum_{k=1}^{n-1} \sharp C e l_{n-2-(k-1)}^{l-2} z^{k}\right) x^{n} \\
& =x+z x^{2} \sum_{n \geq 0}\left(\sum_{k=0}^{n} \sharp C e l_{n-k}^{n} z^{k}\right) x^{n} \\
& =x+z x^{2} \sum_{n \geq 0} P_{n}\left(\frac{1}{y}\right)(x z)^{n}=x+z x^{2} f_{K}\left(x z, \frac{1}{z}\right) .
\end{aligned}
$$

Therefore, we get the following relation verified by $f_{K}$ :

$$
\left((1+z) x^{2}\right) f_{K}^{2}(x, z)+(-1+(2+z) x) f_{K}(x, z)+1=0 .
$$

Finally, the generating series of the Stasheff polytopes verifies

$$
f_{K}(x, z)=\frac{1+(2+z) x-\sqrt{1-2(2+z) x+z^{2} x^{2}}}{2(1+z) x^{2}} .
$$

\section{ACKNOWLEDGEMENTS}

This paper is part of the author's Ph.D. thesis. I would like to thank my advisor Jean-Louis Loday for his confidence. I also wish to thank Benoit Fresse for his help and for the proof of Theorem 1, I am grateful to Birgit Richter and Martin Markl who have carefully read my thesis and to Wee Liang Gan who pointed out some inaccuracies.

\section{REFERENCES}

[Ag1] M. Aguiar, Infinitesimal Hopf algebras, Contemporary Mathematics 267 (2000) 1-30. MR1800704 (2001k:16066)

[Ag2] M. Aguiar, On the associative analog of Lie bialgebras, J. Algebra 244 (2001), no. 2, 492-532. MR 1859038 (2003c:17035)

[Ag3] M. Aguiar, Infinitesimal Hopf algebras and the cd-index of polytopes, Discrete and Computational Geometry 27 (2002), no. 1, 3-28. MR.1871686 (2003b:52004)

[B] J. Beck, Triples, algebras and cohomology, Dissertation, Columbia University, 1967.

[BF] C. Berger, B. Fresse, Combinatorial operad actions on cochains, Math. Proc. Cambridge Philos. Soc. 137 (2004), no. 1, 135-174. MR2075046 (2005e:18013)

[BGS] A. Beilinson, V. Ginzburg, W. Soergel, Koszul duality patterns in representation theory, J. Amer. Math. Soc. 9 (1996), no. 2, 473-527. MR1322847 (96k:17010)

[C] H. Cartan, Algèbres d'Eilenberg-MacLane et homotopie, in "Séminaire Henri Cartan, 1954-55".

[Chas] M. Chas, Combinatorial Lie bialgebras of curves on surfaces, Topology 43 (2004), no. 3, 543-568. MR2041630 (2005b:57038)

[CS] M. Chas, D. Sullivan, Closed string operators in topology leading to Lie bialgebras and higher string algebra, The legacy of Niels Henrik Abel, (2004) 771-784. MR2077595 (2005f:55007) 
[Dr1] V. Drinfeld, Hamiltonian structures on Lie groups, Lie bialgebras and the geometric meaning of classical Yang-Baxter equations, Soviet Math. Dokl. 27 (1983), no. 1, 68-71. MR0688240(84i:58044)

[Dr2] V. Drinfeld, Quantum groups, in Proceedings of the International Congress of Mathematicians, Vol. 1, 2 (Berkeley Calif.), (1986) Providence, RI, (1987), Amer. Math. Soc. 798-820. MR0934283 (89f:17017)

[EE] B. Enriquez, P. Etingof, On the invertibility of quantization functors, J. Algebra 289 (2005), no. 2, 321-345. MR 2142375 (2006b:17024)

[F] T. Fox, The construction of the cofree coalgebras, J. Pure Appl. Algebra 84 (1993), 191-198. MR 1201051 (94e:16044)

[FM] T. Fox, M. Markl, Distributive laws, bialgebras and cohomology, in "Operads: proceedings of renaissance conferences (Hartford/Luminy, 1995)", 167-205, Contemp. Math. 202, Amer. Math. Soc., Providence, RI, (1997). MR.1436921(98a:18006)

[Fr] B. Fresse, Koszul duality of operads and homology of partition posets, in "Homotopy theory: relations with algebraic geometry, group cohomology, and algebraic $K$-theory", 115-215, Contemp. Math., 346, Amer. Math. Soc., Providence, RI, 2004. MR2066499 (2005g:18015)

[G] W. L. Gan, Koszul duality for dioperads, Math. Res. Lett. 10 (2003), no. 1, 109-124. MR1960128 (2004a:18005)

[GJ] E. Getzler, J.D.S. Jones, Operads, homotopy algebra and iterated integrals for double loop spaces, preprint, arXiv:hep-th/9403055 (1994).

[GK] V. Ginzburg, M.M. Kapranov, Koszul duality for operads, Duke Math. J. 76 (1995), 203-272. MR.1301191 (96a:18004)

[Ko] M. Kontsevich, Formal (non) commutative symplectic geometry, The Gelfand Mathematical Seminars, 1990-1992, 173-187, Birkhäuser Boston, Boston, MA, 1993. MR1247289 (94i:58212)

[Kos] J.-L. Koszul, Homologie et cohomologie des algèbres de Lie, C. R. Acad. Sci. Paris 228, (1949). 65-127. MR0036511(12:120g)

[La] F. W. Lawvere, Functorial semantic of algebraic theories, Proc. Nat. Acad. Sci. U.S.A. 50 (1963), 869-872. MR0158921 (28:2143)

[L1] J.-L. Loday, Notes on Koszul duality for associative algebras, preprint http://www-irma.u-strasbg.fr/ loday/PAPERS/koszuldual.ps (1999).

[L2] J.-L. Loday, Dialgebras and Related Operads, Lecture Notes in Mathematics 1763, Springer-Verlag, 2001. MR.1864390 (2002e:00012)

[L3] J.-L. Loday, La renaissance des opérades, Séminaire Bourbaki (Exp. No. 792), Astérisque 237 (1996), 47-74. MR1423619 (98b:18010)

[L4] J.-L. Loday, Scindement d'associativité et algèbres deHopf, to appear in the Proceedings of the Conference in honor of Jean Leray, Nantes (2002). MR2145941 (2006a:16054)

[LR1] J.-L. Loday, M. Ronco, Algèbres de Hopf colibres, C. R. Acad. Sci Paris, Ser. I 337, Ser. I (2003), 153-158. MR2001126 (2004g:16041)

[McCS] J.E. McClure, J.H. Smith, A solution of Deligne's Hochschild cohomology conjecture, Recent progress in homotopy theory (Baltimore, MD, 2000), 153-193, Contemp. Math., 293, Amer. Math. Soc., Providence, RI, 2002. MR1890736 (2003f:55013)

[MacL1] S. Mac Lane, Categories for the working mathematician (second edition), Graduate Texts in Mathematics 5, Springer Verlag, 1998. MR1712872 (2001j:18001)

[MacL2] S. Mac Lane, Categorical algebra, Bull. Amer. Math. Soc. 71, (1965) 40-106. MR0171826 (30:2053)

[Ma1] M. Markl, Cotangent cohomology of a category and deformations, Journal of Pure and Applied Algebra 113 (1996) 195-218. MR.1415558 (98f:18008)

[Ma2] M. Markl, A resolution (minimal model) of the PROP for bialgebras, preprint arXiv:math.AT/0209007 (2002). MR2203621 (2006i:16061)

[Ma3] M. Markl, Distributive laws and Koszulness Ann. Inst. Fourier (Grenoble) 46 (1996), no. 2, 307-323. MR1393517(97i:18008)

[MV] M. Markl, A. A. Voronov, PROPped up graph cohomology, preprint arXiv: math.QA/0307081 (2003).

[May] J. P. May, Definitions: operads, algebras and modules, in "Operads: proceedings of renaissance conferences (Hartford/Luminy, 1995)", 1-7, Contemp. Math. 202, Amer. Math. Soc., Providence, RI, (1997). MR1436912 (97m:18001) 
[Me] S.A. Merkulov, PROP profile of deformation quantization and graph complexes with loops and wheels,preprint, arXiv:math.QA/0412257 (2005).

[Pr] S. Priddy, Koszul resolutions, Trans. Amer. Math. Soc. 152 (1970), 39-60. MR0265437 (42:346)

[S] J.-P. Serre, Gèbres, Enseign. Math. (2) 39 (1993), no. 1-2, 33-85. MR.1225256 (94h:16074)

[V1] B. Vallette, Free monoid in monoidal abelian categories, preprint, http://math. unice.fr/ $\sim$ brunov/publications/FreeMonoid.ps (2004).

[V2] B. Vallette, Dualité de Koszul des props, Ph.D. thesis, preprint, http://math.unice.fr/ $\sim$ brunov/publications/these.ps (2003). MR2096610 (2005g:18016)

[V3] B. Vallette, Homology of generalized partition posets, Journal of Pure and Applied Algebra 208 (2007), no. 2, 699-725. MR2277706

[W] J. A. Weibel, An introduction to homological algebra, Cambridge Studies in Advanced Mathematics 38, Cambridge University Press, 1994. MR.1269324 (95f:18001)

Laboratoire J. A. Dieudonné, Université de Nice, Parc Valrose, 06108 Nice Cedex 02, FRANCE

E-mail address: brunov@math.unice.fr

$U R L$ : http://math.unice.fr/ brunov 